Pacific Journal of Mathematics

iNTRINSIC TRANSVERSALITY STRUCTURES 


\title{
INTRINSIC TRANSVERSALITY STRUCTURES
}

\author{
NORMAN LEVITT AND ANDREW RANICKI
}

This paper introduces the notion of an intrinsic transversality structure on a Poincaré duality space $X^{n}$. Such a space has an intrinsic transversality structure if the embedding of $X^{n}$ into its regular neighborhood $W^{n+k}$ in Euclidean space can be made "Poincaré transverse" to a triangulation of $W^{n+k}$. This notion relates to earlier work concerning transversality structures on spherical fibrations, which are known to be essentially equivalent to topological bundle reductions. Thus, for $n \geq 5$, a Poincaré duality space $X^{n}$ with a transversality structure on its Spivak normal fibration (i.e., with an "extrinsic" transversality structure) is, up to a surgery obstruction, realizable as a topological manifold. An intrinsic transversality structure, however, not only guarantees the existence of an extrinsic transversality structure but gives rise as well to a canonical solution of the resulting surgery problem. Thus, as our main result, an equivalence is obtained between intrinsic transversality structures and topological manifold structures. This yields a number of corollaries, among which the most important is a "local formula for the total surgery obstruction" which assembles this obstruction to the existence of a manifold structure on $X^{n}$ from the local singularities of a realization of the simple homotopy type of $X^{n}$ as a (non-manifold) simplicial complex.

0. Introduction. The aim of this paper is to address and, the authors dare to hope, largely culminate a certain chain of ideas that has recurred, if at times only implicitly, as a sort of subtext in the history of structure theory of manifolds. Roughly stated the principle involved is this: If one studies the category of Poincaré duality spaces, where "equivalence" is usually taken to mean simple homotopy equivalence, and the corresponding bundle theory, i.e., spherical fibrations, then one notes immediately that the analogs of Thom transversality fail within the category. But this failure has, as well, a positive side to it, the point being that when an exception occurs to the general rule that transversality fails, the exception arises because the object studied "lifts" in some sense to the topological category where transversality holds. To take an example essentially due to Sullivan [18], suppose we look at two manifolds $M^{n}$, $M_{0}^{n}$, equivalent in the "Poincaré category," i.e., there is a (simple) homotopy equivalence $f: M_{0}^{n} \rightarrow M^{n}$. "Failure of transversality" in this case may be construed to mean that if we take a submanifold (or more generally, a subvariety) $V \subseteq M^{n}$, then, although $f$ may certainly be made transverse to $V$ via a small deformation, there is no guarantee that 
$f \mid f^{-1} V \rightarrow V$ is a homotopy equivalence, as would be the case for trivial reasons if $f$ were up to homotopy a homeomorphism, i.e, an equivalence in the topological-manifold category. But the celebrated characteristic variety theorem admits of the following interpretation: There is a collection of subvarieties (i.e., manifolds and $\mathbf{Z} / p \mathbf{Z}$ manifolds) $\left\{V_{i}\right\}$ of $M$ so that $f \mid f^{-1} V_{i} \rightarrow V_{i}$ a homotopy equivalence, all $i$, implies the normal invariant of $f$ vanishes and, in consequence, if $M^{n}$ is simply connected, $f$ is homotopic to a homeomorphism.

Similar in spirit is the following result ([7], [1]). Given an arbitrary spherical fibration $\eta^{k}, k \geq 3$ over $B$, consider the Thom space $T\left(\eta^{k}\right)$, and a map $f: M^{n} \rightarrow T\left(\eta^{k}\right)$. If $\eta^{k}$ were an "honest" bundle, (vector, PL, $\widetilde{\mathrm{PL}}$, TOP) then $f$ could be made transverse to $B$ by a small deformation. In the Poincaré category, however, transversality fails; even with $M^{n}$ a manifold, it may not be possible to arrange for $f^{-1} B \subset M$ to be a Poincaré-duality space with normal fibration in $M$ equivalent to $\left(f \mid f^{-1} B\right)^{*} \eta^{k}$. The positive side of this failure is the following: Suppose all such maps can be made Poincaré transverse, in a mutually consistent way. (This acquires a more precise meaning by saying that the map $\mathscr{W}(\eta) \rightarrow$ $T(\eta)$ admits a section where $\mathscr{W}(\eta)$ is the subcomplex of the singular complex of $T(\eta)$ consisting of Poincaré transverse singular simplices. See [4].) Then the conclusion is that $\eta$ admits a TOP structure and, moreover, the "Poincaré transversality structure" actually arose from this bundle structure on $\eta$ via the standard transversality phenomena in the TOP category.

Considerations such as these were often referred to by Sullivan, somewhat whimsically, as the "Winkelnkemper Axiom" in honor of an aphorism coined by $\mathrm{H}$. E. Winkelnkemper in informal conversation. The "axiom" may be stated: "Transversality unlocks the secrets of manifolds".

In the present paper we push this metaphysical principle a bit further. The basic problem we have in hand is the detection and classification of topological manifold structures on a Poincaré duality space. Throughout this paper we assume orientability to simplify the exposition, although our results can certainly be extended to the unorientable case.

Classically, the problem has been thought of as a two-stage problem: Given a Poincaré duality space $X^{n}$, with Spivak normal fibration $\nu$, first check whether $\nu$ has a TOP (resp. PL, O) reduction. If the answer is positive, see whether a reduction exists such that the resulting surgery obstruction in $L_{n}\left(\pi_{1} X\right)$ vanishes. (In the special case $\pi_{1} X=\{1\}$ it is well known that the second problem can be got round, but this fails for arbitrary $\pi_{1} X$.) 
The results alluded to above certainly have a bearing on the first stage of the problem. If we think of $\nu$ as a high, but finite, dimensional spherical fibration, we may ask whether maps $f: M^{n} \rightarrow T(\nu)$ can always be made Poincaré transverse in a mutually consistent way, i.e., does $\mathscr{W}(\nu) \rightarrow T(\nu)$ admit a section, or, to use the terminology of [7], does $\nu$ have a transversality structure. If so, $\nu$ has a TOP reduction, and, obviously, conversely. We note, in passing, our introduction of some new terminology: We say that $X^{n}$ has an extrinsic transversality structure iff $\nu$ has a transversality structure. (To be absolutely precise and consistent with our terminology in $\$ 1$ below, a transversality structure is actually a section of $\mathscr{Y}(\nu) \rightarrow T(\nu)$ where $\mathscr{Y}(\nu) \supseteq \mathscr{W}(\nu)$ is a space reflecting a weaker kind of transversality than true Poincaré transversality, which nonetheless is seen to fail for general spherical fibrations.) Thus $X^{n}$ has a TOP-reducible Spivak normal fibration if and only if it has an extrinsic transversality structure.

Our terminology naturally suggests that there is something we wish to call an intrinsic transversality structure. The notion of intrinsic structure arises from the following considerations. Heretofore, we have looked for Poincaré transversality in maps $f: M \rightarrow T(\eta)$ where $M$ is a true manifold and $\eta$ a mere spherical fibration. But we may also ask a "dual" question. Suppose $f: X^{n} \rightarrow T\left(\xi^{k}\right)$ is a map where $X^{n}$ is merely a Poincaré duality space and $\xi^{k}$ a true bundle (TOP, PL, O) over $B$. Can $f$ be made transverse, i.e., up to homotopy and, avoiding certain mild technicalities, is it true that $f^{-1} B$ is a sub-Poincaré-duality space of $X^{n}$ with "tubular neighborhood" $\left(f \mid f^{-1} B\right)^{*} \xi^{k}$ ? In general, the answer is "no," even when $\xi^{k}$ is a trivial bundle. More generally, if $Q$ is a PL-stratified space, e.g., a triangulated PL manifold, the problem of making $f: X^{n} \rightarrow Q$ "Poincaré transverse" to the stratification is, in general, insoluble. However, if $X^{n}$ has a manifold structure, then it obviously can be solved. This suggests a "metaphysical," or, to put it less politely, over-vague notion of intrinsic transversality for $X^{n}$, namely, that all such maps $f: X^{n} \rightarrow Q$ may be made Poincaré-transverse to the stratification of the range $Q$, in a "consistent" way. We make this more precise, however, by picking essentially one such map to one such stratified space. Let $W^{n+k}$ denote a regular neighborhood of $X^{n}$ in Euclidean space $R^{n+k}$, and let it be combinatorially triangulated. We then ask whether the natural homotopy equivalence $\iota: X^{n} \rightarrow W^{n+k}$ may be made Poincare transverse to the triangulation. If so, we say that $X^{n}$ admits an intrinsic transversality structure. (More precisely, an intrinsic transversality structure is an equivalence class, in a sense to be defined in $\S 1$ below, of transverse maps homotopic to $\iota$.) 
Now it was noted in [7] in the absence of the current terminology, that if $X^{n}$ admits an intrinsic transversality structure, then the Spivak fibration $\nu$ admits a transversality structure, (i.e., $X^{n}$ admits an extrinsic transversality structure) and hence $\nu$ has a topological reduction. Thus the remaining obstruction to putting a manifold structure on $X^{n}$ is a surgery obstruction in $L_{n}\left(\pi_{1} X^{n}\right)$. What was overlooked in [7], and which, indeed, is the heart of the present paper, is that the topological reduction of $\nu$ arising from an intrinsic transversality structure on $X^{n}$ leads to a surgery problem with vanishing obstruction. Moreover, not only does the obstruction to the surgery vanish but, assuming $n \geq 5$, there is a canonical way of actually doing the surgery.

Thus the primary result, stated here with some terminology still undefined and more precisely as Theorem 1.27 below:

THEOREM. Intrinsic transversality structures on $X^{n}$ are in one-to-one correspondence with $\mathscr{S}_{\mathrm{TOP}}\left(X^{n}\right)=$ topological manifold structures on $X^{n}$.

The plan of the paper is as follows: In $\$ 1$ we give a brief review of results to date (in precise terminology) and after suitable definitions have been made prove the result just stated as Theorem 1.27, leaving incomplete certain details concerning the algebraic surgery spaces $\mathbf{L}_{*}(G)$ and the assembly map. We also prove the relatively minor extensions of 1.27.

In \$2 we give some further applications; in particular, some necessary and sufficient conditions for making a manifold into a covering space are announced as well as some results on decomposing manifolds as Cartesian products.

In $\$ 3$ we apply the missing details concerning surgery spaces and the assembly map. In particular, we give a brief exposition of the notion of quadratic Poincaré complex (QAPC), a category the understanding of which is vital to our methods of proof. Indeed, one must go further: Our whole approach depends on the insight that surgery problems may be "algebraicized," i.e., may be represented on the "chain level" by QAPC's whose bordism type is the classical surgery obstruction element, while at the same time, the QAPC category is itself "quasi-geometric" in that those objects are amenable to the "cut and paste" techniques which one associates with geometric topology. Thus one may algebraicize without losing track of the "schematic diagrams" so useful in picturing the topology of manifolds.

Finally, $\S 3$ concludes with some further observations on the algebraic nature of the structure set $\mathscr{S}_{\text {TOP }}\left(X^{n}\right)$ of manifold structures on $X^{n}$. We show as well that the total surgery obstruction of Ranicki [13] may be 
characterized on the "cycle" level as the obstruction to the existence of an intrinsic transversality structure. That is, if we take an arbitrary embedding $X^{n} \subset W^{n+k}$ and measure with sufficient insight its failure to be Poincaré transverse to a given triangulation of $W^{n+k}$ we obtain a representative on the cycle level of the total surgery obstruction. We then briefly indicate how, given a specific triangulation of $X$ itself as a simplicial complex, this leads to a "local combinatorial formula" for the total surgery obstruction where in one adds up, in a suitable sense, the failure of links of simplices to be homology spheres to obtain a representative of the total surgery obstruction.

1. Intrinsic and extrinsic transversality structures. For the moment, we consider (finite) Poincaré-duality spaces, within the meaning of the following definition

1.1. Definition. A (finite) Poincaré-duality space of formal dimension $n$ is a finite CW complex $X$ such that if $W^{n+k}$ is a compact, codimension-0 submanifold of $S^{n+k}, k \geq 3$, with $W^{n+k}$ homotopically equivalent to $X$, and $\pi_{1} \partial W \rightarrow \pi_{1} W$ an isomorphism, then the inclusion $\partial w \rightarrow W$ has $S^{k-1}$ as its homotopy-theoretic fiber.

Likewise, $(X, \partial X)$ is an $n$-dimensional Poincaré pair iff, given $W^{n+k}$ a codimension-0 submanifold of $D^{n+k}, \quad k \geq 3$, and $V^{n+k-1}=W^{n+k} \cap$ $S^{n+k-1}$ (a codimension-0 submanifold of $\left.\partial W^{n+k}\right)$ such that $(W, V)$ is the homotopy type of $(X, \partial X)$, and $\pi_{1} \partial V \rightarrow \pi_{1} V, \pi_{1}(\partial W-\operatorname{int} V) \rightarrow \pi_{1} W$ are isomorphisms, then the inclusion $\partial W-$ int $V \subseteq W$ has fiber $S^{k-1}$, as does $\partial V \subseteq V$ with

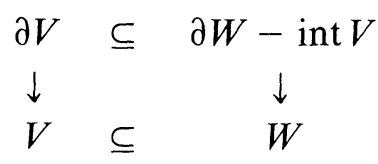

a map of $(k-1)$-spherical fibrations.

We use the abbreviations PD-space and PD-pair for Poincaré duality spaces and pairs respectively.

If $X$ is an $n$-dimensional PD-space then, with $W^{n+k}$ as in the preceding definition, the $(k-1)$-spherical fibration $\partial W^{n+k} \subseteq W^{n+k}$ determines a unique stable spherical fibration $\nu$ called the Spivak normal fibration of $X$. If $X, \partial X$ is a $P$-pair, then with $W^{n+k}, V^{n+k-1}$ as above, $(\partial W-$ int $V) \subseteq W$ similarly determines a unique stable fibration over $X$. Now let $\eta$ denote a $(k-1)$-spherical fibration over some arbitrary base space $B, \eta: S(\eta) \rightarrow B$. The Thom space $T(\eta)$ is: $\mathscr{M}(\eta) \cup c S(\eta)(\mathscr{M}(\eta)$ $=$ mapping cylinder of $\eta$ ).

Let $M$ be a compact PL manifold. 
1.3. Definition. A map $f: M \rightarrow T(\eta)$ is said to be Poincaré Transverse Regular (PT) iff $P=f^{-1} M(\eta), Q=f^{-1} c S(\eta)$ are codimension-0 submanifolds of $M$ meeting in $f^{-1} S(\eta)=\partial P=\partial Q$ with

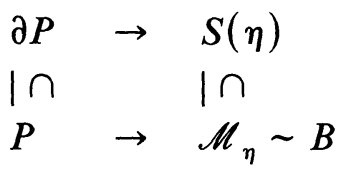

a map of $(k-1)$-spherical fibrations.

If this is the case, then $P$ is an $n-k$ dimensional Poincaré space with Spivak normal fibration $(f \mid P)^{*} \eta \oplus \nu_{M} \mid P$, where $\nu_{M}$ denotes the stable (PL) normal bundle of $M$; likewise, if $M^{m}$ is compact with boundary $\partial M$, then the condition that $f: M^{m} \rightarrow T(\eta)$ be PT is that:

(1) $f \mid \partial M$ is PT

(2) $P=f^{-1}(\mathscr{M}(\eta)), Q=f^{-1}(c S(\eta))$

are codimension- 0 submanifolds meeting in $f^{-1}(S(\eta))=\overline{\partial P-\partial M}$, a codimension- 0 submanifold of $\partial P$, so that the diagram

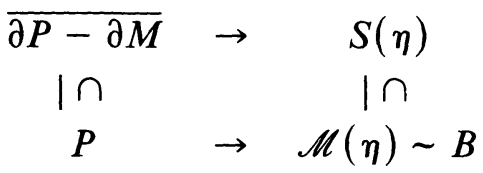

is a map of $(k-1)$-spherical fibrations. If we set $\Delta=\partial P-\operatorname{int} f^{-1}(S(\eta))$ $=(f \mid \partial M)^{-1}(\mathscr{M}(\eta))$, then clearly $(P, \Delta)$ is a Poincaré pair.

Consider the standard $q$-simplex $\Delta^{q}$, and a singular $q$-simplex of $T(\eta), \sigma: \Delta^{q} \rightarrow T(\eta)$.

1.4. Definition. $\sigma$ is said to be a PT simplex if $\sigma$ is a PT map as are all the faces of $\sigma$.

The PT simplices form a subcomplex $\mathscr{W}(\eta)$ of the singular complex $S(T(\eta)) \sim T(\eta)$, so that there is, in effect, a natural map $\mathscr{W}(\eta) \stackrel{\rho}{\rightarrow} T(\eta)$. Given a simplicial complex $K$ and a map $f: K \rightarrow T(\eta), f$ factors up to homotopy, through $\mathscr{W}(\eta)$ if and only if it is possible to deform $f$ so as to be PT on every simplex of $K$.

Generalizing, we see that a homotopy class of sections $\mathscr{W}(\eta) \stackrel{\rho}{\rightleftarrows} T(\eta)$ yields a way of deforming arbitrary maps $f: K \rightarrow T(\eta)$ to maps ${ }^{s}$ PT on each simplex. This is unique in the sense that if $f_{1}, f_{2}$ are the results of two such possible deformations, then they are homotopic through a homotopy which is PT on each simplex of some triangulated homotopy cylinder.

In [7], a result was given which claimed that, given a 4-connected base space, $X$, and a $k$-dimensional spherical fibration $\eta$ over $X$, homotopy 
classes of sections of $\mathscr{W}(\eta) \rightarrow T(\eta)$ [i.e. transversality structures on $\eta$ ] are in one-one correspondence with topological reductions of $\eta$.

In [1] this result was somewhat improved in that the connectivity assumption on $X$ was reduced from 4-connected to 1-connected. However the reader should be aware that in [1] the space $\mathscr{W}(\eta)$ is replaced, in the statement of the theorems, by a somewhat smaller subspace consisting of PT singular simplices of $T(\eta)$ which are, first of all small with respect to a certain open covering of $T(\eta)$. Moreover, it is also assumed that for simplices $\sigma^{k+2}$ in this subcomplex the inverse image of $M(\eta)$ under $\sigma$ : $\Delta^{k+2} \rightarrow T(\eta)$ is homotopy equivalent to a 2-manifold (with boundary homotopy equivalent to $\sigma^{-1}\left(M(\eta) \subseteq \Delta^{k+2}\right)$.

Our first aim is to give stronger versions of this result and, in particular, to study the case when $\pi_{1} B$ is aribtrary. We contend that the most perspicacious way of looking at things involves shifting focus from the study of the fiber of $\mathscr{W}(\eta) \rightarrow T(\eta)$ and considering instead a map $s$ from $T(\eta)$ to a certain space $\mathbf{L}_{-k-1}\left(\pi_{1} B\right)$ (depending only on the fundamental group of the base). $\mathbf{L}_{-k-1}\left(\pi_{1} B\right)$ is a $k$-connected space with the property that $\pi_{k+r} \mathbf{L}_{-k-1}\left(\pi_{1} B\right)$ is the Wall group $L_{r-1}\left(\pi_{1} B\right)$. Simultaneously, we shall define $\bar{s}: T(\eta) \rightarrow \mathbf{L}_{-k-1}(\{1\})$ and this map turns out to be of primary significance for our subsequent results.

In the construction of this map, and the determination of its fiber, it will be necessary to consider a weaker property (in fact several weaker properties) for maps to $T(\eta)$ than the PT property defined above.

As a preliminary step, we consider normal transversality (NT) for maps of manifolds to $T(\eta)$.

1. Definition. If $M^{m}$ is an (oriented) manifold we shall say that $f$ : $M^{m} \rightarrow T(\eta)$ is NT iff $f^{-1}(\mathscr{M}(\eta))=U$ is a codimension-0 submanifold of $M^{m}$ with boundary $V=f^{-1}(S(\eta))$ so that $U$ is of homotopy dimension $m-k$, and so that the homomorphism

$$
\bigcap[U]: H^{m-k}(U, \mathbf{Z}) \rightarrow H_{0}(U, \mathbf{Z})
$$

is an isomorphism, where $U=f^{*} \Phi(\eta) \cap[U, V], \Phi(\eta)$ is the integral Thom class of $T(\eta), f^{*} \Phi(\eta)$ its pullback to $H^{k}(U, V)$ and $[U, V]$ the $m$-dimensional orientation class of $(U, \partial U)=(U, V)$.

The definition of the NT property for the case when $M^{m}$ has non-void boundary is the obvious generalization of the property above. As it turns out, the NT property is a rather weak one, in the sense that any map may be deformed to an NT map, and two NT maps which are homotopic may be connected by an NT homotopy. 
If $f: M^{m} \rightarrow T(\eta)$ is an NT map, we shall call $U=f^{-1}(\mathscr{M}(\eta))$ and $\eta$-normal subspace of $M^{m}$. If $M^{m}=S^{m}$ we shall simply call $U$ an $\eta$-normal space. (The notion of normal space is due to Quinn [11].)

We may define an NT singular simplex of $T(\eta)$ in analogy to PT simplices. The collection of NT simplices form a subcomplex of the singular complex of $T(\eta)$. However, since the NT property is, in homotopic terms, a "generic" one, it is easily seen that the subcomplex of NT simplices may simply be identified, up to homotopy, as $T(\eta)$ itself.

Now consider the following property, which we label ST

1.6. Definition. A map $f: M^{m} \rightarrow T(\eta)\left(M^{m}\right.$ oriented, as before) is said to be ST iff $f$ is NT and, moreover, $U=f^{-1}(\mathscr{M}(\eta))$ has the property that

$$
\bigcap[U]: H^{m-k-j}(U, \mathbf{Z}) \rightarrow H_{j}(U, \mathbf{Z})
$$

is an isomorphism for all $j$.

Here it must be emphasized that the coefficients for homology and cohomology are simple. In other words, we have $U$ a "Poincaré duality space" of dimension $m-k$ in the naive sense that cohomology in dimension $m-k-j$ is "Poincaré dual" to homology in the complementary dimension $j$. Of course, if $\pi_{1} U=\{1\}$ this says that $U$ is a P.D. space in our original sense and, of course, if $U$ is an orientable PD-space in our original sense then it certainly has this property. Note, however, that if $\pi_{1}(U) \neq\{1\}$, it does not necessarily follow that $U$ is a PD-space. In particular, it may not be the case that $V \subseteq U$ is, up to homotopy, a $(k-1)$-spherical fibration. Thus PT implies ST but not conversely. As before, we may take the subcomplex of ST singular simplices, denoted by $Z(\eta)$, and we have $W(\eta) \subseteq Z(\eta)$.

On the other hand, consider once more an NT map $f: M^{m} \rightarrow T(\eta)$. In [16, §7.3] (refining an idea of Quinn [11]) there is associated to $U=f^{-1}(M(\eta))$, the given $\eta$-normal subspace of $M^{n}$, a quadratic algebraic Poincaré complex [Q.A.P.C.] $Q_{f}$ of dimension $n-1$ over the ring $\mathbf{Z}\left(\pi_{1} B\right)(B=$ base of $\eta)$. Recall [14] that Q.A.P.C.'s form a bordism category. That is, one may define the notion of $n$-dimensional Q.A.P.C.with-boundary (over a given ring), whose boundary is an $(n-1)$ dimensional Q.A.P.C. (without boundary). Thus, one readily defines bordism between two such objects. Since "disjoint union" and " $\times I$ " operations are defined, bordism classes of $n$-dimensional complexes form a group, and, in fact, the group of bordism classes of $n$-dimensional Q.A.P.C.'s (over $\mathbf{Z}(\pi)$ ) may be identified with the Wall group $L_{n}(\pi)$. 
Now if the NT map $f: M^{m} \rightarrow T(\eta)$ is, in fact, PT, it is seen that $Q_{f}$ is the null Q.A.P.C. The converse is more-or-less true, that is, (assuming $m-k \geq 5$ ), if $Q_{f}$ is Q.A.P.C. bordant to zero, then $f$ may be deformed to a PT map. We shall not be using the converse, however, as we cannot actually prove it.

This leads to the notation of QT maps. Specifically,

1.7. Definition. A QT map of $M^{m}$ to $T(\eta)$ is a pair $(f, J)$ where $f$ is an NT map $f: M^{m} \rightarrow T(\eta)$ and $J$ is a Q.A.P.C. bordism (over $\mathbf{Z}\left(\pi_{1} B\right)$ ) of $Q_{f}$ to zero.

The definition of a QT map when $M^{m}$ has a boundary is a bit more complicated. We begin by observing that if $n$-dimensional Q.A.P.C.'s with boundary $Q_{1}, Q_{2}$ have common boundary $\partial Q_{1}=\partial Q_{2}$ then we may form the "union" $Q_{1} \cup_{\partial} Q_{2}$, which turns out to be a "closed" $n$-dimensional Q.A.P.C. (i.e., without boundary).

Now if $f: M^{m} \rightarrow T(\eta)$ is an NT map, then we obtain a Q.A.P.C.with-boundary $Q_{f}$ such that $\partial Q_{f}=Q_{f / \partial M}$, as might be expected. Thus we may define a $Q T$ map of $M^{m}$ to $T(\eta)$ as a triple $(f, K, J)$ where $f$ : $M^{m} \rightarrow T(\eta)$ is an NT map, $K$ is a bordism of $Q_{f \mid \partial M}$ to zero, and $J$ is a bordism of $Q_{f} \cup_{\text {a }} K$ to zero.

When we try to define a QT singular simplex of $T(\eta)$ the situation becomes still more involved. We base our definition on the observation that if $Q_{1}, Q_{2}$ are Q.A.P.C.'s with boundary, $\partial Q_{1}=R_{1} \cup_{\partial} S, \partial Q_{2}=R_{2}$ $\cup_{\partial} S$, then we may form $Q=Q_{1} \cup_{S} Q_{2}$ having $\partial Q=R_{1} \cup_{\partial} R_{2}$. With this in mind, we may specify what is meant by an $m$-dimensional QT simplex. We start off with an $m$-dimensional NT simplex $\sigma: \Delta^{m} \rightarrow T(\eta)$. For each $i$-dimensional face $\tau$ of $\sigma$, we have the $i-k$-1-dimensional Q.A.P.C. $Q_{\tau}$, such that $\partial Q_{\tau}=\cup_{\rho} Q_{\rho}$ where $\rho$ ranges along the $(i-1)$ dimensional faces of $\tau$ and the union identifies a "piece" of $\partial Q_{\rho_{1}}$ with the "piece" of $\partial Q_{\rho_{2}}$ piece corresponding to $\rho_{1} \cap \rho_{2}$.

The additional data on $\sigma$ which makes it into a QT simplex has the following form:

First, for $\operatorname{dim} \tau=k$ we have a 0 -dimensional bordism $K_{\tau}$ of $Q_{\tau}$ (i.e. the null Q.A.P.C.) to zero.

This means that, for $\operatorname{dim} \tau=k+1$ we have a "closed" 0-dim Q.A.P.C. $K_{\tau}^{\prime}=Q_{\tau} \cup \cup_{\rho<\tau ; \operatorname{dim} \rho=k} K_{\rho}$. We then choose a 1-dimensional bordism $K_{\tau}$ of $K_{\tau}^{\prime}$ to zero.

Now, for $\operatorname{dim} \tau=k+2$, we obtain a closed 1-dimensional Q.A.P.C. $K_{\tau}^{\prime}=Q_{\tau} \cup_{\partial} \bigcup_{\rho<\tau ; \operatorname{dim} \rho=k+1} K_{\rho}$. So we choose a 2-dimensional bordism $K_{\tau}$ of $K_{\tau}^{\prime}$ to zero. 
We continue in this way up to $\tau=\sigma$. Thus, a QT $m$-simplex is an NT simplex $\sigma$, together with the lattice of data $\left\{K_{\tau}\right\}$ modeled on the combinatorial structure of the standard $m$-simplex $\Delta^{m}$.

The set of QT simplices from an s.s. complex $\mathscr{X}(\eta)$ which maps, forgetfully, into $T(\eta)$.

Consider, moreover, a PT map $M^{m} \rightarrow T(\eta)$. As was observed previously, $Q_{f}$ is the null Q.A.P.C. so by choosing the null bordism, of $Q_{f}$ to zero one obtains, in a trivially canonical way, a QT map of $M$ to $T(\eta)$.

By the same token, there is a canonical way of assigning to each PT singular simplex of $T(\eta)$ a corresponding QT singular simplex thus giving rise to: $\mathscr{W}(\eta) \rightarrow \mathscr{X}(\eta)$.

There remains one more notion of transversality to explore which is, as we shall see, the weakest yet the most significant for our purpose. First, consider the fact that, given a homomorphism of $G \rightarrow H$, one obtains a covariant functor from Q.A.P.C.'s over $\mathbf{Z}(G)$ to Q.A.P.C.'s over $\mathbf{Z}(H)$. In particular, if $f: M^{m} \rightarrow T(\eta)$ is NT, we may consider $R_{f}$, the Q.A.P.C. over $\mathbf{Z}=\mathbf{Z}(1)$ which is the image of $Q_{f}$ under the functor induced by $\pi_{1} B \rightarrow\{1\}$. We may readily define

1.8. Definition. An RT map is a pair $(f, J)$ where $f: M^{m} \rightarrow T(\eta)$ is $\mathrm{NT}$ and $J$ is a Q.A.P.C. bordism (over $\mathbf{Z}$ ) of $R_{f}$ to zero.

The notion of an RT-singular simplex is readily obtained by a straightforward modification of the definition of QT-simplex, and we thus obtain an s.s. complex $\mathscr{Y}(\eta)$ of RT-simplices and a forgetful map $\mathscr{X}(\eta)$ $\rightarrow \mathscr{Y}(\eta)$

We remark that $R_{f}$ has a certain geometric significance. If $f: M^{m} \rightarrow$ $T(\eta)$ is ST, it is seen that $R_{f}$ is the null Q.A.P.C. over Z. Again, a converse of sorts holds: assuming $m-k \geq 5$, if $R_{f}$ is bordant to zero, then $f$ deforms to an ST map. Thus, an ST map $f: M^{m} \rightarrow T(\eta)$ gives rise to an RT map (by taking, as additional data, the null bordisms of $R_{f}$ to zero) and we thereby obtain a map $\mathscr{Z}(\eta) \rightarrow \mathscr{Y}(\eta)$. Putting all these constructions together we have a diagram

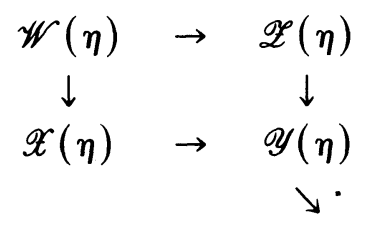

$T(\eta)$

reflecting the fact that PT is a stronger than ST or QT, both of which are stronger than RT. 
We may now relate the $\mathscr{X}(\eta) \rightarrow T(\eta)$ (resp., $\mathscr{Y}(\eta) \rightarrow T(\eta)$ ) to a certain map $T(\eta) \rightarrow \mathbf{L}_{-k-1}\left(\pi_{1} B\right)$ (resp. $T(\eta) \rightarrow \mathbf{L}_{-k-1}(\{1\})$ ) where $\mathbf{L}_{-k-1}(G)$ is a certain space, depending only on the group $G$, such that

(1) $\mathbf{L}_{-k-1}(G)$ is $k$-connected,

(2) $\pi_{k+r}\left(\mathbf{L}_{-k-1}(G)\right)=L_{r-1}(G)$ for $r \geq 1$, with $\Omega \mathbf{L}_{-k-1}(G)=\mathbf{L}_{-k}(G)$.

In particular, we may specify the space $\mathbf{L}_{-k-1}(G)$ as an s.s. complex, having one $r$-simplex for $0 \leq r \leq k$, and, for $r>k, r$-simplices are as follows: A $k+1$-simplex is simply a 0 -dimensional Q.A.P.C. over $\mathbf{Z}(G)$; a $k+2$-simplex $\sigma$ is a collection $\left\{Q_{\tau}\right\}$ of 0 -dimensional Q.P.C.'s, one for each $(k+1)$ face $\tau$ of the standard $(k+2)$-simplex, together with a 1-dimensional Q.A.P.C. $Q_{\sigma}$ with $\partial Q_{\sigma}=\cup Q_{\tau}$.

Continuing in this way suppose we have defined an $(r-1)$-skeleton of $\mathbf{L}_{-k-1}(G)$ so that for each simplicial map $f$ of $S^{r-1}$ to the $(r-1)$ skeleton we obtain an $(r-k-1)$-dimensional Q.A.P.C. (without boundary) $Q_{f}$. We then may define a typical $r$-simplex $\sigma$ of $\mathbf{L}_{-k-1}(G)$ to be a simplicial map $f: \dot{\Delta}^{r} \rightarrow(r-1)$-skeleton together with a bordism $Q_{\sigma}$ of $Q_{f}$ to zero.

Now clearly, if we have simplicial map $f$ of $S^{r}$ to the newly-defined $r$-skeleton, we may define $Q_{f}$ as $\cup_{\rho} Q_{\rho}$, where $\rho$ ranges over the simplicies of $S^{r}, Q_{\rho}=Q_{\operatorname{im} \rho}$ and appropriate identifications (in the world of Q.A.P.C.'s) are made under this union.

It is not hard to see that $\pi_{k+r}\left(\mathbf{L}_{-k-1}(G)\right)=$ the bordism classes of $(r-1)$-dimensional Q.A.P.C.'s over $\mathbf{Z}[G]=L_{r-1}(G)(r \geq 1)$.

Now when $G=\pi_{1} B, B=$ base $\eta$ we easily see how the map $T(\eta) \rightarrow$ $\mathbf{L}_{-k-1}\left(\pi_{l} B\right)$ is constructed. First of all, we think of $T(\eta)$ as the s.s. complex of NT singular simplices. On the $k$-skeleton of $T(\eta)$ the definition is the unique possible simplicial map. Now consider a $(k+1)$-simplex $\sigma$ of $T(\eta)$. To it we assign the $(k+1)$-simplex $\rho$ of $\mathbf{L}_{-k-1}\left(\pi_{1} B\right)$ given by $Q_{\rho}=Q_{\sigma}$, where $Q_{\sigma}$ is the Q.A.P.C. (bordant to 0 ) defined by the NT map $\sigma$.

Continuing, to the $(k+2)$-skeleton, for a typical $(k+2)$-simplex $\sigma$, we have $Q_{\sigma}$ a bordism to zero of $\bigcup_{\tau<\sigma ; \operatorname{dim} \tau=k+1} Q_{\tau}$. Moreover, the map $\dot{\boldsymbol{\sigma}} \rightarrow \mathbf{L}_{-k-1}\left(\pi_{1} B\right)$ already defined is simplicial and the Q.A.P.C. it defines is exactly $\cup_{\tau} Q_{\tau}$. So specifying $Q_{\sigma}$ gives us a $k+2$-simplex $\rho$ in $\mathbf{L}_{-k-1}\left(\pi_{1} B\right)$ which we take to be the image of $\sigma$.

Continuing in this way, suppose the map has been defined as the $(r-1)$-skeleton of $T(\eta)$ so that, for any $r$-simplex $\sigma$ of $T(\eta)$, the Q.A.P.C. induced by $\dot{\sigma} \rightarrow \mathbf{L}_{-k-1}\left(\pi_{1} B\right)$ is exactly the Q.A.P.C. $Q_{\dot{\mathbf{\sigma}}}$. Then, the Q.A.P.C.-with-boundary $Q_{\sigma}$ specifies an $r$-simplex $\rho$ of $\mathbf{L}_{-k-1}\left(\pi_{1} B\right)$ and we let $\rho$ be the image of $\sigma$. Henceforth, let us designate the natural map $\mathscr{X}(\eta) \rightarrow T(\eta)$ by $q$ and $T(\eta) \rightarrow \mathbf{L}_{-k-1}\left(\pi_{1} B\right)$ by $s$. 
Moreover, we easily observe that a homomorphism $G_{1} \rightarrow G_{2}$ induces a simplicial map $\mathbf{L}_{-k-1}\left(G_{1}\right) \rightarrow \mathbf{L}_{-k-1}\left(G_{2}\right)$. Thus, in particular, we have $c$ : $\mathbf{L}_{-k-1}\left(\pi_{1} B\right) \rightarrow \mathbf{L}_{-k-1}(\{1\})$. Let $\bar{s}=c \circ s: T(\eta) \rightarrow \mathbf{L}_{-k-1}(\{1\})$. Finally let $r: \mathscr{Y}(\eta) \rightarrow T(\eta)$ denote the natural map.

1.9. THEOREM. The following sequences are fibrations

$$
\begin{aligned}
& \mathscr{X}(\eta) \stackrel{q}{\rightarrow} T(\eta) \stackrel{s}{\rightarrow} \mathbf{L}_{-k-1}\left(\pi_{1} B\right) \\
& \mathscr{Y}(\eta) \stackrel{r}{\rightarrow} T(\eta) \stackrel{\bar{s}}{\rightarrow} \mathbf{L}_{-k-1}(\{1\}) .
\end{aligned}
$$

The proof is an easy exercise in standard s.s. techniques.

The next result seems to be the most elegant generalizations of [7], [1], et al. First of all, we define a transversality structure in the following way:

1.10. Definition. A transversality structure on an oriented spherical fibration $\eta^{k}$ is a fiber homotopy class of sections $u$ of $r$

$$
\begin{gathered}
\mathscr{Y}(\eta) \\
r \downarrow \uparrow u \\
T(\eta)
\end{gathered}
$$

N.B. This is, a priori, a weaker notion than that used in [4] where a transversality structure was a section of $\mathscr{W}(\eta) \rightarrow T(\eta)$. Here, we think of a transversality structure on $\eta$ as a systematic way of deforming an arbitrary map $K \rightarrow T(\eta)$ ( $K$ a simplicial complex) to a map which is RT on all simplices (hence, $\mathrm{RT}$ on $K$ if $K$ is a manifold).

1.11. THEOREM. Transversality structures on $\eta$ are in 1-1 correspondence with TOP reductions of $\eta$, i.e. fiber homotopy clases of liftings

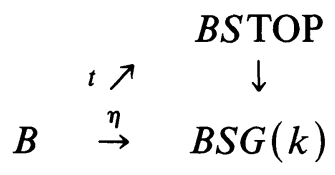

The proof is essentially the same as that outlined in [7] and carried through in [1]. One shows inductively that if there is a lifting of $\eta$ to BSTOP over the $j$ skeleton of $B$, there is a section of $t: \mathscr{Y}(\eta) \rightarrow T(\eta)$ over the $j+k$-skeleton of $T(\eta)$, and conversely. Then, one sees that the primary obstruction to extending the lift to the $(j+1)$-skeleton of $B$, (which lies in $H^{j+1}\left(B, \pi_{j}(G /\right.$ TOP $)$ ) and the primary obstruction to extending the section to the $(j+k+1)$-skeleton of $T(\eta)$ (which lies in

$$
\begin{aligned}
& \tilde{H}^{j+k+1}\left(T(\eta), \pi_{j+k+1}\left(\mathbf{L}_{-k-1}(\{1\})\right)\right) \\
& \left.\quad=\tilde{H}^{j+k+1}\left(T(\eta), L_{j}(\{1\})\right)=\tilde{H}^{j+k+1}\left(T(\eta), \pi_{j} G / \text { TOP }\right)\right)
\end{aligned}
$$


exactly correspond under the Thom isomorphism. The result is thus an easy exercise in elementary obstruction theory, provided one can see precisely how $\pi_{*} G /$ TOP is related to surgery obstructions. It is worth noting, in passing, that the construction of $\mathscr{Y}(\eta)$ and the introduction of the notion of an RT map are chiefly motivated as a means of understanding in its most precise form the relation between "transversality" and topological bundle structures on spherical fibrations. It will be remembered that what Brumfiel and Morgan show explicitly in [1] is that for spherical fibrations $\eta$ with simply connected base space, and for a suitably chosen subcomplex $\overline{\mathscr{W}}(\eta) \subset \mathscr{W}(\eta)$, homotopy classes of sections of $\overline{\mathscr{W}}(\eta) \rightarrow T(\eta)$ are in 1-1 correspondence with topological reductions of $\eta$. It is further possible to show (though all the technical details have not yet been written down) that $\overline{\mathscr{W}}(\eta) \subset \mathscr{W}(\eta)$ is a homotopy equivalence. We shall leave these arguments aside as not strictly relevant to the results to be proved below. Suffice it to say however, that the role the assumption of simple connectivity of the base $B$ of $\eta$ is merely to assure that the obstructions arising in the problem of finding a section of $\overline{\mathscr{W}}(\eta) \rightarrow T(\eta)$ are identifiable with elements in $H^{i+1}\left(B, \pi_{i}(G /\right.$ TOP $\left.)\right)$ (i.e., the relative homotopy group $\pi_{i+k-1}(T(\eta), \overline{\mathscr{W}}(\eta))$ is identified with $\pi_{i}(G / \mathrm{TOP}), k=\operatorname{dim} \eta$ ). When $\mathscr{Y}$ is substituted for $\overline{\mathscr{W}}$ (or $\mathscr{W}$ ), it is virtually automatic that this identification may be made. Thus, under the much weaker assumption that $\eta$ merely be orientable, made throughout this paper, the Thom isomorphism argument outlined above connecting the problems of finding on the one hand a lifting for

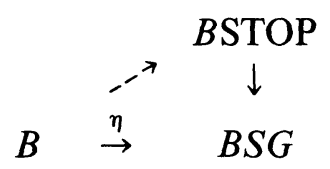

and on the other, a section of $\overline{\mathscr{W}}(\eta) \rightarrow T(\eta)$ is easily made to go through. To put it another way, the natural diagram

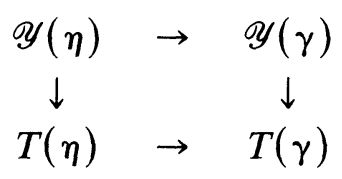

is always a map of fibrations, irrespective of $\pi_{1}(B)$, with $\gamma$ the universal oriented $(k-1)$-spherical fibration over $B S G(k)$.

It is important, moreover, to point out precisely how ths correspondence between topological reductions of $\eta$ and transversality structures on $\eta$ is induced. The point is that given a TOP structure on $\eta$ (assume, now that $k \geq 5$ ), we may then take the evaluation map from the singular complex $S(T(\eta))$ onto $T(\eta)$ and deform it to a map which is, in a 
sufficiently good sense, topologically transverse regular. The qualification arises because of the possibility that, after deformation into the desired map $f$, we may have, for some $(k+4)$-simplices $\sigma$ of $S(T(\eta))$, the space $f^{-1}(\sigma) \subseteq M$ a homology manifold, rather than a topological manifold (see [17]). However, a map topologically transverse, even in this slightly qualified sense, is obviously PT (hence QT, RT and ST). This deformation of the canonical map $S(T(\eta)) \rightarrow T(\eta)$ may, of course, be thought of as a well-defined section of $r: \mathscr{Y}(\eta) \rightarrow T(\eta)$. Hence we see that a Top reduction of $\eta$ yields a transversality structure: The point of the theorem is to demonstrate that the converse holds.

We now specialize to the case where $B$ is replaced by $X$, an $n$-dimensional Poincaré duality space $(n \geq 5)$, and $\eta$ by some $k$-dimensional representative $\nu$ of the Spivak normal fibration of $X$ (we assume $k>n$ ).

1.12. Definition. An extrinsic transversality structure on $X$ is a transversality structure on $\nu$.

We shall shortly contrast this kind of structure on $X$ with another (to be called, naturally, an intrinsic transversality structure). The point of the distinction to be made is in connection with the theory of topological manifold structures on P.D. spaces. Obviously, by dint of Theorem 1.11, extrinsic transversality structures on $X$ are to be identified with TOP reductions of $\nu$. From the point of view of classical surgery theory, then, extrinsic transversality structures on $X$ say a good deal, but certainly not everything, about manifold structures on $X$. In particular, if $X$ is 1-connected, then an extrinsic transversality structure, i.e., a TOP reduction of $\nu$ gives rise to a surgery problem, but it is well known that $X$ has a topological manifold structure, when $n$ is even, even if the resulting surgery obstruction fails to vanish. In other words, an extrinsic transversality structure guarantees at least one manifold structure, but there are very possibly more extrinsic transversality structures than manifold structures when $n$ is even.

In the non-simply-connected case, we cannot even say this much: We may very well have an extrinsic transversality structure without any clear way of obtaining a manifold structure, because there is in general no way to get around the non-vanishing of the non-simply-connected surgery obstruction that arises.

Moreover, given a single topological manifold structure on $X$, extrinsic transversality structures may be identified with $[X, G / \mathrm{TOP}]$ and the relation of extrinsic transversality structures to the set $\mathscr{S}(X)$ of manifold structures on $X$ is then the problematical one given by the Sullivan-Wall 
structure sequence

$$
[\Sigma X, G / \mathrm{TOP}] \rightarrow L_{n+1}\left(\pi_{1} X\right) \rightarrow \mathscr{S}(X) \rightarrow[X, G / \mathrm{TOP}] \rightarrow L_{n}\left(\pi_{1} X\right) .
$$

The notion of "intrinsic transversality structure" that we are going to propound has the very desirable property that intrinsic transversality structures on $X$ are (exactly!) in 1-1 correspondence with manifold structures on $X$. We begin with a philosophical apology for introducing this notion. First of all, we take note of what Theorem 1.11 says about fibrations $\eta$. If there is a "systematic" way of deforming an arbitrary map $f: M \rightarrow T(\eta)$ ( $M$ a PL manifold) to a map which is PT (or, even more weakly, namely RT) then $\eta$ is realizable as a TOP bundle and the "system" is equivalent (in the world of RT maps) to the one which arises by exploiting topological transversality theory. I.e., if $\eta$ has a "transversality property" in the Poincaré-duality-space then $\eta$ is a bundle, and that property merely reflects the underlying bundle structure. However, there is an additional "transversality property" for manifolds which is not wholly detected merely by examining the "transversality properties" of the normal bundle. To take a simple example, if $\theta^{j}$ is, say, a vector bundle over $B$, and we have a map $f: M \rightarrow T\left(\theta^{j}\right)$, then, of course, we may deform $f$ to a transverse regular map $g$, so that $g^{-1} B$ is a codimension $j$ submanifold of $M$ with a normal bundle induced from $\theta^{j}$. (If $M$ is merely a TOP manifold, we may, of course, have to insist that $\operatorname{dim} M-j \neq 4$ or allow $g^{-1} B$ to be a homology manifold if $\operatorname{dim} M-j=4$; but this is a minor point.) It should be noted that how this is done reflects the manifold structure on $M$ and not merely the underlying homotopy type (i.e. PD-space structure).

Thus, we might ask whether, given a PD-space $X^{n}$ and map $f$ : $X \rightarrow T(\theta)$, we can "deform" $f$ to a "transverse regular" map in the following sense.

1.13. Definition. We say that $f: X^{n} \rightarrow T\left(\theta^{j}\right)$ is strictly $p T$ iff $f^{-1}(B)=Y^{n-j}$ is a P.D. space, $f^{-1}\left(D\left(\theta^{j}\right)\right)=D\left((f \mid Y)^{*} \theta^{j}\right)$ with $f$ being a disc-bundle map on this subspace, and $f^{-1} c S\left(\theta^{j}\right), f^{-1}\left(S\left(\theta^{j}\right)\right)$ an $n$-dimensional P.D. pair.

Thus, if $f$ is pT, $X^{n}$ "splits" into two P.D. pairs $f^{-1}\left(D\left(\theta^{j}\right)\right)$, $f^{-1}\left(S\left(\theta^{j}\right)\right), f^{-1} c S\left(\theta^{j}\right), f^{-1}\left(S\left(\theta^{j}\right)\right)$ where the first piece is a "regular neighborhood" of the Poincaré "subspace" $Y^{n-J}$, mapping via a bundle map into $D\left(\theta^{j}\right)$.

1.14. Definition. We say that $f: X^{n} \rightarrow T\left(\theta^{j}\right)$ is deformable to a $\mathrm{pT}$ map iff there is an $n$-dim P.D. space $X_{1}^{n}$, a simply homotopy equivalence 
$h: X^{n} \rightarrow X_{1}^{n}$, and a strictly pT map $g: X_{1}^{n} \rightarrow T\left(\theta^{j}\right)$ so that

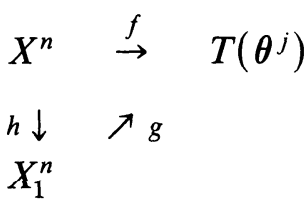

is homotopy commutative.

If we ask whether any map $f: X^{n} \rightarrow T\left(\theta^{j}\right)$ is deformable to a pT one, in the above sense, the answer is "no". In particular, if $\theta^{j}$ is merely the trivial $j$-dimensional bundle over a point, a positive answer would imply that the P.D.-bordism theory of a space is a homology theory, which is not true; excision fails, and excision is no more than the deformability property specified above.

The general principle which we are going to make quite precise, shortly, is that if there is a way of deforming maps from $X^{n}$ to Thom spaces, as well as more general stratified spaces, to "Poincaré transverse" maps, then $X^{n}$ is in fact a manifold. In fact, there is one particular stratified space and one particular map which tells the whole story, so far as manifold structure theory on $X^{n}$ is concerned.

Again, we are obliged to consider several different notions of "transversality." Also, we modify somewhat the underlying geometric situation in that, rather than considering maps from a P.D. space to the Thom space of a bundle, we consider maps to a triangulated manifold and consider what it might mean for the map to be transverse to the triangulation. Thus, let $X^{n}$ be an $n$-dimensional orientable P.D. space and $W^{j}$ a $j$-dimensional, combinatorially-triangulated manifold.

1.15. Definition. $f: X^{n} \rightarrow W^{j}$ is said to be normally transverse (nT) to the triangulation iff $f^{-1} \partial W=\varnothing$ and, for each simplex $\sigma^{j-k}$ of $W^{j}$, $\boldsymbol{\sigma} \nsubseteq \partial W,\left(f^{-1} \boldsymbol{\sigma}, f^{-1} \dot{\boldsymbol{\sigma}}\right)$ is a $\nu$-normal pair of dimension $n-k$, where $\nu$ is the Spivak normal fibration of $X^{n}$.

1.16. Definition. $f: X^{n} \rightarrow W^{j}$ is said to be deformable to an $\mathrm{nT}$ map iff there exists a P.D. space $X_{1}^{n}$, a homotopy equivalence $h: X^{n} \rightarrow X_{1}^{n}$ and an nT map $g: X_{1}^{n} \rightarrow W^{j}$ such that the diagram

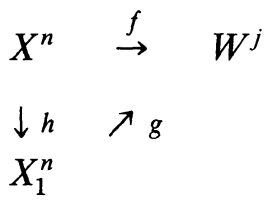

is homotopy commutative. 
We assert, omitting the proof, that any map $f: X^{n} \rightarrow W^{j}$ is deformable to an nT map.

1.17. Definition. The map $f: X^{n} \rightarrow W^{j}$ is said to be

(a) pT iff $f^{-1}(\partial W)=0$ and, for each $\sigma^{j-k}$ of $W$ with $\sigma \nsubseteq \partial W$, $\left(f^{-1} \boldsymbol{\sigma}, f^{-1} \dot{\boldsymbol{\sigma}}\right)$ is an orientable P.D. pair of dimension $n-k$

(b) sT if $f^{-1}(\partial W)=0$ and $\left(f^{-1} \sigma, f^{-1} \dot{\sigma}\right)$ is a pair satisfying $(n-k)$ dimensional Poincaré-Lefschetz duality for simple integral coefficients i.e., there is a class $\left[X_{\sigma}\right] \in H_{n-k}\left(f^{-1} \sigma, f^{-1} \dot{\sigma} ; \mathbf{Z}\right)$ so that in the diagram (all coefficients $\mathbf{Z}$ )

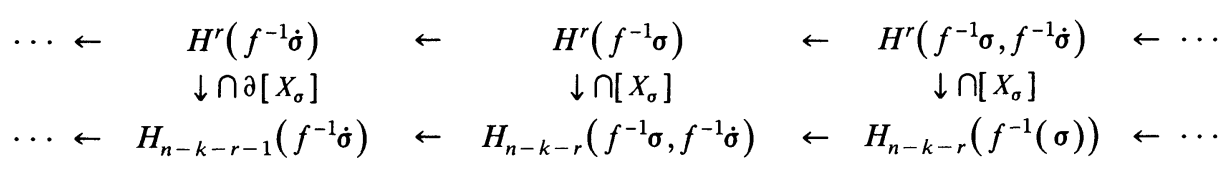

all vertical arrows are isomorphism.

Now note that if $f: X^{n} \rightarrow W^{j}$ is an nT map, then, for each $\sigma^{j-k}$ of $W^{j}$, we obtain a Q.A.P.C.-with-boundary $Q_{\sigma}$ of dimension $n-k-1$ over $\mathbf{Z}\left(\pi_{1} X^{n}\right)$ (arising from the bundle map $\nu \mid f^{-1} \sigma \rightarrow \nu$ ).

1.18. Definition. A qT map of $X^{n} \stackrel{\prime}{\rightarrow} W^{j}$ consists of a strictly nT map $f: X^{n} \rightarrow W^{j}$ together with a set $\left\{J_{\sigma}\right\}$ of Q.A.P.C.'s-with-boundary over $\mathbf{Z}\left(\pi_{1} X\right)$ one for each $\sigma^{j-k} \nsubseteq \partial W, \operatorname{dim} J_{\sigma}=n-k$, such that $\partial J_{\sigma}=$ $Q_{\sigma} \cup \cup_{\tau<\sigma} J_{\tau}$.

If we have an nT map $f: X^{n} \rightarrow W^{j}$, let $\bar{Q}_{\sigma}$ be the Q.A.P.C. over $\mathbf{Z}(\{1\})$ which is the functorial image of $Q_{\sigma}$. We then have

1.19. Definition. An rT map of $X^{n}$ to $W^{j}$ is an nT map $f$ : $X^{n} \rightarrow W^{j}$ together with a set $\left\{J_{\sigma}\right\}$ of Q.A.P.C.'s-with-boundary over $\mathbf{Z}\{1\}$, one for each $\sigma^{j-k} \nsubseteq \partial W, \operatorname{dim} J_{\sigma}=n-k$ such that $\partial J_{\sigma}=\bar{Q}_{\sigma} \cup$ $\bigcup_{\tau<\sigma} J_{\tau}$.

Clearly, if $f: X^{n} \rightarrow W^{j}$ is $\mathrm{pT}$, it is $\mathrm{nT}$, and, since $Q_{\sigma}$ identically the null-Q.A.P.C. for all $\sigma$, we automatically obtain a qT map by taking $J_{\sigma}$ to be the null Q.A.P.C. Analogously, if $f: X^{n} \rightarrow W^{j}$ is sT it is nT and we then automatically obtain an rT map in the same fashion as above. It is obvious that pT implies sT and that there is a canonical way of associating an rT map to a qT map.

We take note of the fact that the notion of $\mathrm{nT}$ map makes sense when $X^{n}$ is not a P.D. space but merely an $n$-dimensional normal space with orientable normal fibration $\nu$. By the same token, let $X^{n}$ now be a normal 
space with a normal map

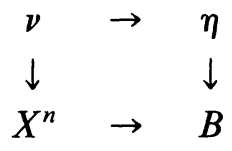

As we have seen in this situation, we obtain a Q.A.P.C. $Q_{X}$ over $\mathbf{Z}\left(\pi_{1} B\right)$ of dimension $n-1$. Thus, if $f: X^{n} \rightarrow W^{j}$ is an nT map, we obtain a system $\left\{Q_{\sigma}\right\}$ of Q.A.P.C.'s-with-boundary over $\mathbf{Z}\left(\pi_{1} B\right)$, one for each simplex $\sigma^{j-k} \nsubseteq \partial W$, so that $\bigcup_{\sigma} Q_{\sigma}$ is canonically chain homotopy equivalent to $Q_{X}$. We may then define a qT map as an nT-map $f: X^{n} \rightarrow W^{j}$ together with a collection $\left\{J_{\sigma}\right\}$ of Q.A.P.C.'s-with-boundary over $\mathbf{Z}\left(\pi_{1} B\right)$ so that $\partial J_{\sigma}=Q_{\sigma} \cup \cup_{\tau<\sigma} J_{\sigma}$ just as in the case when $X^{n}$ is a P.D. space and $\eta=\nu$. In the same manner, an rT map will have the Q.A.P.C.'s $J_{\sigma}$ with $\mathbf{Z}(\{1\})$ as ground ring and $\bar{Q}_{\sigma}$ will replace $Q_{\sigma}$ in the definition.

Let $X^{n}$ once more denote a P.D. space:

1.20. Definition. We say that $f: X^{n} \rightarrow W^{n}$ is deformable to

(i) a pT map

(ii) an sT map

(iii) a qT map

(iv) an rT map

if and only if there is a P.D. space $X_{1}^{n}$, a homotopy equivalence $h$ : $X_{1}^{n} \rightarrow X_{1}^{n}$, and a map $g: X_{1}^{n} \rightarrow W$ making

$$
\begin{array}{lll}
X^{n} & \stackrel{f}{\rightarrow} & W^{j} \\
\downarrow h & \nearrow g \\
X_{1}^{n} &
\end{array}
$$

homotopy commutative, such that

(i) $g$ is $\mathrm{pT}$

(ii) $g$ is sT

(iii) $g$ is $\mathrm{nT}$ and can be provided with a collection $\left\{J_{\sigma}\right\}$ of Q.A.P.C.'s over $\mathbf{Z}\left[\pi_{1} X\right]$ ( $\sigma$ a simplex of $\left.W^{j}\right)$ so that $\left(g\left\{J_{\sigma}\right\}\right)$ is a qT map.

(iv) $g$ is $\mathrm{nT}$ and can be provided with a collection $\left\{J_{\sigma}\right\}$ of Q.A.P.C.'s over $\mathbf{Z}(\{1\})$ making $\left(g,\left\{J_{\sigma}\right\}\right)$ an rT map.

Obviously, for a given map $f$, deformability to a $\mathrm{pT}$ map implies deformability to an sT map and to a qT map, both of which imply deformability to an rT map.

As we have hinted earlier, there is a special class of manifolds $W$ and maps $f: X^{n} \rightarrow W$ for which deformability to a pT map, or even to an rT map, takes on a special significance. We shall start with a fairly rough 
statement giving the general idea and subsequently refine it to give the theorem in its stronger final form.

Rough Statement: Let $W^{n+k},(n \geq 5, k$ large) be a triangulated regular neighborhood of $X^{n}$ in $\mathbf{R}^{n+k}$. $X^{n}$ admits a topological manifold structure if and only if the inclusion $i: X^{n} \subseteq W^{n+k}$ is deformable to a pT map.

We shall work in the direction of strengthening this result by weakening the deformability condition to something weaker than $\mathrm{pT}$ and by actually obtaining a classification theorem for manifold structures on $X^{n}$.

The key observation is the following: Let $W^{n+k}$ be the triangulated regular neighborhood of $X^{n}$ in $\mathbf{R}^{n+k}$.

1.21. Lemma. Suppose $i: X^{n} \subseteq W^{n+k}$ is deformable to an rT map. Then the Spivak normal fibration $\nu\left(X^{n}\right)$ admits a TOP reduction.

Proof. Replace $i$ by an rT map $f$ and then, since $k$ is large assume that $f$ is an embedding, i.e., $f$ embeds $f^{-1}(\sigma)$ in $\sigma$ for every simplex $\sigma$ of $W^{n+k}$. Identify $X$ with its embedded image, and let $X_{\sigma}=X \cap \sigma$. Take a smaller regular neighborhood $U$ of $X$ such that $U \cap \sigma$ is a regular neighborhood of $X_{\sigma}$. Now the $k$-dimensional representative $\nu^{k}$ of the Spivak normal fibration of $X^{n}$ can be thought of as $\partial U \subseteq U$, and so $T\left(\nu^{k}\right)$ may be thought of as $U / \partial U$. Now consider the collapsing map $W \rightarrow$ $U / \partial U=T\left(\nu^{k}\right)$ which is the identity on int $U$ and takes everything else to the base point. Obviously, if we take $W \cup c \partial W=T^{\prime}$ we may extend to $c$ : $T^{\prime} \rightarrow T\left(\nu^{k}\right)$. Now, with the obvious extension of the triangulation of $W$ to one on $W \cup c \partial W=T^{\prime}$, we see that the map $c$ is RT on each simplex of $T^{\prime}$. (In fact, if $f$ is pT (resp. sT, qT), then $f$ is a fortiori PT (resp. ST, QT)). But then, if $T^{\prime}$ is given a simplicial partial ordering (i.e. an ordering of the vertices linear on simplices) we obtain a lifting $\gamma: T^{\prime} \rightarrow \mathscr{Y}\left(\nu^{k}\right)$. But note that $T^{\prime}$ may also be thought of as $T\left(\nu^{k}\right)$ and $c: T^{\prime} \rightarrow T\left(\nu^{k}\right)$ may be identified up to homotopy, with the identity. Thus $\gamma$ is a section of $\mathscr{Y}\left(\nu^{k}\right) \rightarrow T\left(\nu^{k}\right)$ and defines a TOP reduction of $\nu^{k}$. Note that the class of this reduction does not depend on the simplicial ordering.

Thus we are part of the way towards the proof of our Rough Statement; if $i: X^{n} \subseteq W^{n+k}$ is deformable to a pT (hence to an rT) map $\nu^{k}\left(X^{k}\right)$ has a TOP reduction. The question, then, is one of seeing why the surgery obstruction associated to such a reduction should vanish.

The idea, then, is to see at precisely what level the surgery obstruction is defined. The standard way of proceeding, of course, is to take the TOP 
reduction into account by deforming the map $f: S^{n+k} \rightarrow T\left(\nu^{k}\right)$ (representing the generator of $\left.\tilde{H}_{n+k}\left(T\left(\nu^{k}\right)\right)\right)$ to a map topologically transverse to $X^{n}$, using the specific topological structure on $\nu^{k}$. If $f^{-1}\left(X^{n}\right)=M^{n}$, the degree one normal map $M^{n} \rightarrow X^{n}$ then defines a surgery element in $L_{n}\left(\pi_{1} X\right)$ which does not depend on $M^{n}$ per se, since $M^{n} \rightarrow X^{n}$ is well-defined up to a cobordism of normal maps into $X^{n}$. First, we make note of a minor fact. If we think, as before, of $T\left(\nu^{k}\right)$ as having the homotopy type, in a standard way, of the triangulated regular neighborhood $W^{n+k}$ with the cone on $\partial W$ attached, we may think of $M^{n} \rightarrow X^{n}$ as having arisen from deforming the $W \cup c \partial W \rightarrow T\left(\nu^{k}\right)$ to a topologically transverse map (remembering that $W \subset c \partial W$ is a manifold away from the cone point). In fact, this map may be made topologically transverse (in the sense of Scharlemann [17]) on each simplex of $W \cup c \partial W$.

On the other hand, our major point is that if the natural homotopy equivalence of $W \cup c \partial W$ with $T\left(\nu^{k}\right)$ is represented by a map $W \cup c \partial W$ $\rightarrow T\left(\nu^{k}\right)$ which is merely RT on each simplex, there is a definition of the surgery obstruction which avoids the necessity of going through the procedure above, which, to outline it briefly once more, involved interpreting the RT-map $W \cup \partial W \rightarrow T\left(\nu^{k}\right)$ as a section of $\mathscr{Y}\left(\nu^{k}\right) \rightarrow T\left(\nu^{k}\right)$, yielding a TOP reduction of $\nu^{k}$, which, in turn, leads to a surgery problem via the standard Browder-Novikov transversality construction, and thence to an element of $L_{n}\left(\pi_{1} X\right)$. Rather the following is true.

1.22. LeMMA. Let $g: W \cup c \partial W \rightarrow T\left(\nu^{k}\right)$ be an RT map consistent with the usual homotopy identification of $W \cup c \partial W$ with $T\left(\nu^{k}\right)$. Then there is a well-defined ( up to chain-homotopy) Q.A.P.C. $\tilde{\Phi}_{g}$ over $\mathbf{Z}\left[\pi_{1} X\right]$. Moreover, if $g$ is homotopic to the RT map $g^{\prime}: W \cup c \partial W \rightarrow T\left(\nu^{k}\right)$ via the RT-homotopy $G:(W \cup c \partial W) \rightarrow T\left(\nu^{k}\right)$, then there is a well-defined Q.A.P.C.-with boundary $\tilde{\Phi}_{G}$ with $\partial \tilde{\Phi}_{G}=\tilde{\Phi}_{g} \cup\left(-\tilde{\Phi}_{g^{\prime}}\right)$. Thus, there is a natural assignment from RT-homotopy classes of such maps to $L_{n}\left(\pi_{1} X\right)$.

Finally, $\left[\tilde{\Phi}_{g}\right] \in L_{n}\left(\pi_{1} X\right)$ identical to the surgery obstruction element realized by taking the TOP reduction of $\nu^{k}$ induced by $g$ and then taking the obstruction for the associated surgery problem.

Details will be provided later, but we here make a brief plausibility argument. First, by way of illustrating the basic idea in a slightly different context, consider a degree one normal map of, say, PL manifolds $f$ : $M^{n} \rightarrow N^{n}$ and a specific PL triangulation of $N^{k}$. Let $\sigma^{j}$ be a simplex of $N^{k}$, and let $M_{\sigma}^{j}=f^{-1} \sigma^{j}$ be its inverse image, which, clearly may be assumed to be a manifold with boundary $\partial M_{\sigma}^{j}=f^{-1} \dot{\boldsymbol{\sigma}}_{j}$. Obviously, from the fact that $f$ is a normal map, we obtain a normal map $M_{\sigma}^{j}, \partial M_{\sigma}^{j} \rightarrow \sigma^{J}$, 
$\dot{\sigma}^{j} \cong D^{j}, S^{j-1}$. Studying this normal map in isolation we find there is a "kernel complex" which measures the failure of $M^{j}, \partial M^{j} \rightarrow D^{j}, S^{j-1}$ to be a homotopy equivalence i.e., a Q.A.P.C.-with boundary $\Phi_{\sigma}$ of dimension $j$. Note that $\Phi_{\sigma}$ is defined as a Q.A.P.C. over the ring $\mathbf{Z}=\mathbf{Z}[\{1\}]$. Now note that we may "assemble" the various Q.A.P.C.'s $\Phi_{\sigma}$ to form a "global" Q.A.P.C. $\Phi(f)$. That is, for each face relation $\tau<\sigma$, there is a way of identifying $\Phi_{\tau}$ with a "piece" of $\Phi_{\sigma}$, and we may let $\Phi(f)$ denote the union of the $\Phi_{\sigma}$ over all simplices $\sigma$ of $N$. Now, a priori $\Phi(f)$ is an $n$-dimensional Q.A.P.C. (without boundary, assuming $N, M$ are boundaryless) over $\mathbf{Z}[\{1\}]$. But the crucial point is that in the course of this assembly operation, a $\mathbf{Z}\left[\pi_{1} N\right]$ action may be introduced to enrich the structure of $\Phi(f)$ and thereby produce a $Z\left[\pi_{1} N\right]$-Q.A.P.C. $\tilde{\Phi}(f)$. I.e., $\tilde{\Phi}(f)$ measures the failure of $f$ to be a $Z\left[\pi_{1} N\right]$-module equivalence, while $\Phi(f)$ only reflects integral, simple coefficient homology. Thus $\Phi(f)$ is the natural image of $\tilde{\Phi}(f)$ under the forgetful functor form $\mathbf{Z}\left[\pi_{1} N\right]$-Q.A.P.C.'s to Z-Q.A.P.C.'s. More importantly, $[\tilde{\Phi}(f)] \in L_{n}\left(\pi_{1} N\right)$ is precisely the classical Wall surgery obstruction for the normal map $M^{n} \rightarrow N^{n}$ and $[\Phi(f)]$ the simply-connected obstruction associated to it.

Continuing our outline, we replace the triangulation of $N^{n}$ by a triangulation of $W^{n+k}$, where $W^{n+k}$ is, as usual, a regular neighborhood of $N^{n}$ in $\mathbf{R}^{n+k}$. Think of $M^{n} \rightarrow N^{n}$ as having arisen by deforming $W \cup c \partial W \stackrel{\text { id }}{\rightarrow} W \cup c \partial W$ (preserving the cone point) to some arbitrary self map $\hat{f}: W \cup c \partial W \hookleftarrow$ so that $\hat{f}$ is tranverse-regular to $N^{n}$ on each simplex $\sigma$ of $W \cup c \partial W$. (We assume, without loss of generality, that $f^{-1} N^{n} \cap \sigma=$ 0 for all $\sigma$ containing the cone point.) Thus $M^{n}=\hat{f}^{-1}\left(N^{n}\right) \rightarrow N^{n}$ is a degree one normal map. Now suppose $M_{\sigma}^{j}=M^{n} \cap \sigma^{j+k}$ where $\sigma^{j+k}$ is an arbitrary simplex of $W$. Then we still may think of $M^{j}$ as having a "framed" normal bundle by virtue of a well-defined normal map to $N^{n}$ which factors through the contractible space $\sigma$. Thus, for each $M_{\sigma}^{j}$ we have a $j$-dimensional Q.A.P.C.-with boundary $\Phi_{\sigma}$ over the $\operatorname{ring} \mathbf{Z}=\mathbf{Z}\{1\}$, and, as before, we may take $\Phi(f)$ as $\cup_{\sigma} \Phi_{\sigma} /$ (identifications). In this case as well, $\Phi(f)$ pulls back to $\tilde{\Phi}(f)$ over $\mathbf{Z}\left[\pi_{1} N\right]$, and $[\tilde{\Phi}(f)]$ is the surgery obstruction for $f$.

Now, if we think of a P.D. space $X^{n}$ in place of the manifold $N^{n}$, and we imagine that the map $\hat{f}: W \cup c \partial W \hookleftarrow$ is merely PT (rather than PL transverse) on each simplex $\sigma$, the picture remains basically unaltered. that is, for each $\sigma$, we obtain the Q.A.P.C.-with-boundary $\Phi_{\sigma}$ over $\mathbf{Z}$ which measures the failure of $M^{j}, \partial M^{j}$ (now merely a P.D.-pair) to be $D^{j}$, $S^{j-1}$, and we may assemble to obtain $\Phi(f)$, and in fact $\tilde{\Phi}(f)$, over $\mathbf{Z}\left[\pi_{1} X\right]$, which measures the failure of $M^{n}=\hat{f}^{-1}\left(X^{n}\right) \rightarrow X^{n}$ to be a $\mathbf{Z}\left[\pi_{1} X\right]$ equivalence. (Note that here $M^{n}$ is merely a P.D.-space with a 
degree-one normal map to $X^{n}$.) Moreover, $[\tilde{\Phi}(f)] \in L_{n}\left(\pi_{1} X\right)$ is, again, to be identified with the (well-defined) surgery obstruction element for the degree-one normal map $M^{n} \rightarrow X^{n}$.

We carry our generalization one step further. Suppose $\hat{f}: W \cup c \partial W \hookleftarrow$ is merely RT (rather than PT). Then $\left(\hat{f}^{-1}\left(\sigma^{j+k}\right), \hat{f}^{-1}\left(\dot{\sigma}^{j+k}\right)\right)$ is a $\nu(X)$ normal pair, its failure to satisfy simple coefficient Poincaré duality is measured by a $(j-1)$-dimensional Q.A.P.C.-with-boundary over $\mathbf{Z}, \Psi_{\sigma}$, but, as part of the RT structure on $\hat{f}$, we have a specific Q.A.P.C. $\theta_{\sigma}$ with $\partial \theta_{\sigma}=\psi_{\sigma} \cup \rho_{\sigma}$ (the union along $\partial \rho_{\sigma}=\partial \psi_{\sigma}=\psi_{\dot{\sigma}}$ ). Here, $\rho_{\sigma}=\bigcup_{\tau<\sigma} \theta_{\tau}$. We may think, on the algebraic level, of $\theta_{\sigma}$ as an algebraic procedure for connecting $C\left(\hat{f}^{-1}(\sigma)\right), C\left(\hat{f}^{-1}(\dot{\boldsymbol{\sigma}})\right)$ to a pair of chain complexes $\bar{C}_{\sigma}, \partial \bar{C}_{\sigma}$ with an algebraic Poincaré duality structure and, even though geometric "normal data" does not exist for such objects, we still obtain a "kernel complex" measuring the failure of $\bar{C}_{\sigma}, \partial \bar{C}_{\sigma}$ to be homologous to $D^{j}, S^{j-1}$, i.e., a well-defined Q.A.P.C.-with boundary $\Phi_{\sigma}$ over Z . Now, since part of the structure of an RT map is that the data $\left\{\theta_{\sigma}\right\}$ cohere to match the combinatorial structure of $W \cup c \partial W$ we may "assemble" a Poincaré-duality algebraic chain complex $\bar{C}(\hat{f})=\cup_{\sigma} \bar{C}_{\sigma}$ (mod identifications) and a degree-one map $\bar{C}(\hat{f}) \rightarrow C\left(X^{n}\right)$ whose kernel complex admits the structure of a Q.A.P.C. $\Phi(\hat{f})$ over $\mathbf{Z}$. But, in fact, in assembling $\bar{C}(\hat{f})$ a $\mathbf{Z}\left[\pi_{1} X\right]$ action may be introduced to put a $\mathbf{Z}\left[\pi_{1} X\right]$-module structure $\tilde{C}(\hat{f})$ on $\bar{C}(f)$, for which structure $\tilde{C}(f)$ is a Poincaré duality chain complex over $\mathbf{Z}\left[\pi_{1} X\right]$. Simultaneously $\Phi(\hat{f})$ becomes equipped with a $\mathbf{Z}\left[\pi_{1} X\right]$ structure $\tilde{\Phi}(\hat{f})$, measuring the failure of $\tilde{C}(\hat{f}) \rightarrow C(\tilde{X})$ to be a $\mathbf{Z}\left[\pi_{1} X\right]$ chain equivalence. If we let the $g$ of Lemma 1.22 be our $\hat{f}$, then we have defined $\tilde{\Phi}(g)$ by the procedure above. Our discussion implicitly sketches the following corollary to 1.22 .

1.23. COROLlaRY. If the $g$ of 1.22 is, in fact, simplex-wise PT and $Y^{n}=g^{-1} X^{n}$ is the corresponding PD-space then $\tilde{\Phi}(g)$ is, up to chain homotopy, in fact the well-defined kernel complex obstruction of the degreeone normal map

$$
Y^{n} \rightarrow X^{n}
$$

Returning now to the proof of the Rough Statement, we stand in the following position. Given a strictly pT embedding $X^{n} \rightarrow W^{n+k}$, as we have seen, we obtain a map $W \cup c \partial W \rightarrow \mathscr{Y}(\nu)$ (at least, if the triangulation of $W \cup c \partial W$ is ordered), which may be interpreted as a section of the natural map $\mathscr{Y}(\nu) \rightarrow T(\nu)$. Now, given that homotopy classes of sections of this map are in 1-1 correspondence with topological bundle 
structures on $\nu$, we note further that the correspondence in one direction (from TOP bundle structure to sections) may be constructed by taking the homotopy equivalence $W \cup c \partial W \stackrel{h}{\rightarrow} T(\nu)$ and using the explicit bundle structure on $\nu$ to deform it to a map $j$ simplex-wise topologically transverse to the 0 -section $X$. Assuming that $h$ has been so deformed, it is clear that, up to homotopy, a section of $\mathscr{Y}(\nu) \rightarrow T(\nu)$ has been constructed. Moreover, we note that if $M^{n}$ denotes $j^{-1} X$ (and we may assume that $M^{n} \subseteq W \subseteq W \cup c \partial W$ ) then there is a degree-one normal map $f$ : $M^{n} \rightarrow X^{n}$ which is, in fact, a representative of the classical surgery problems which arises in connection with putting a topological manifold structure on $X^{n}$ corresponding to the given topological reduction of $\nu$.

Now suppose the TOP reduction of $\nu$ has arisen via the hypothesis of the Rough Statement, i.e, from the pT embedding $X^{n} \subseteq W^{n+k}$. Then the simplex-wise topologically transverse (hence PT) map $W \cup c \partial W \stackrel{j}{\rightarrow} T(\nu)$ arising from this structure on $\nu$ must be homotopic, through RT maps, to the map $W \cup c \partial W \stackrel{h}{\rightarrow} T(\nu)$, which was PT since it was constructed directly from the pT-embedding $X^{n} \rightarrow W^{n+k}$. That is, we may find a triangulated $(W \cup c \partial W) \times I$ and an RT map $(W \cup c \partial W) \times I \rightarrow T(\nu)$ so that, on one end, we have $j$ and on the other $h$. Now note that the surgery obstruction corresponding to $h$ is zero, since $h$ merely extends the identity id: $X^{n} \rightarrow X^{n}$. We wish to conclude that the same is true of the surgery obstruction $\sigma\left(M^{n} \rightarrow X^{n}\right)$ corresponding to $j$, but by virtue of 1.21 , and 1.22 we see that $\sigma\left(M^{n} \rightarrow X^{n}\right)=\sigma\left(X^{n} \stackrel{\text { id }}{\rightarrow} X^{n}\right)=0$. Thus we may do topological surgery on $f: M^{n} \rightarrow X^{n}$ to convert it into a simple homotopy equivalence.

We wish to strengthen this result, i.e., to convert it into a true classification theorem. To that end we begin with a definition.

1.24. Definition. A $p$-structure of codimension $k$ on $X^{n}$ is an equivalence class of pT embeddings $e: X^{n} \rightarrow W^{+k}$ ( $W^{n+k}$ triangulated).

Equivalence of two such, say $e, e^{\prime}$ means the following:

(a) There is a $\nu$-normal space $N^{n+1}$ with boundary $\partial N=X^{n} \cup\left(-X^{n}\right)$ and an rT embedding $f: N \rightarrow W \times I$, where $W$ is triangulated so that the respective ends $W \times\{0\}, W \times\{1\}$ are triangulated appropriate to $e, e^{\prime}$ respectively and so that $f$ is $e: X \rightarrow W \times\{0\}$ in one copy of $X$ and $e^{\prime}$ : $X \rightarrow W \times\{1\}$ on the other.

(b) Noting that the embedding $f$ defines an RT map $\hat{f}:(W \cup c \partial W)$ $\times I \rightarrow T(\nu)$; that this produces an $(n+1)$-dimensional Q.A.P.C. $\tilde{\Phi}(\hat{f})$ over $\mathbf{Z}\left[\pi_{1} X\right]$ (see 1.21) and that the boundary $\partial \tilde{\Phi}(\hat{f})$ is trivial (since 
$\partial \tilde{\Phi}(\hat{f})=\tilde{\Phi}(\hat{e}) \cup\left(-\tilde{\Phi}\left(\hat{e}^{\prime}\right)\right)$ and $\tilde{\Phi}(\hat{e}), \Phi\left(\hat{e}^{\prime}\right)$ arise from the identity homotopy equivalence $\left.X^{n} \rightarrow X^{n}\right)$ we require that $[\tilde{\Phi}(\hat{f})] \in L_{n+1}\left(\pi_{1} X\right)$ be in the image of $\left[\sum X, G / \mathrm{TOP}\right]$.

We call an equivalence class of $p$-structures an intrinsic transversality structure.

1.25. Note. It will be seen that it is actually no strengthening of the second condition to require that $[\tilde{\Phi}(\hat{f})]$ actually be trivial in $L_{n+1}\left(\pi_{1} X\right)$.

Let $\operatorname{IT}^{k}(X)$ denote the set of codimension $k$ intrinsic transversality structures on $X$. It will be noted, without proof, that there is a suspension map $\operatorname{IT}^{k}(X) \rightarrow \operatorname{IT}^{k+1}(X)$, so that we may speak, if we wish, of stable intrinsic transversality structure, $\operatorname{IT}(X)=\lim \operatorname{IT}^{k}(X)$. However, it will be seen that, for $k$ large, $\operatorname{IT}^{k}(X) \cong \operatorname{IT}^{k+1}(X)$ under suspension, so the distinction is unimportant.

1.26. Remark. Of course, the most obvious example of an intrinsic transversality structure comes from considering a manifold $M^{n}$ (say, smooth or PL for the sake of argument) and a regular neighborhood $W^{n+k}$ of $M^{n}$ in $\mathbf{R}^{n+k}$. If we triangulate $W$, then we may embed $M^{n}$ transverse to the triangulation. Moreover, two such embeddings with respect possibly to different triangulations may be connected by embedding $M \times I$ in a suitably triangulated $W \times I$. One point of the classification theorem about to be stated is that the transversality structure thereby defined is, in fact, a complete invariant of the topological structure which $M^{n}$ imposes on the underlying simple homotopy type.

1.27. TheOREM. For large $k$, there is a bijection $\operatorname{IT}^{k}\left(X^{n}\right) \rightarrow \mathscr{S}_{\mathrm{TOP}}\left(X^{n}\right)$ (where $\mathscr{S}_{\text {TOP }}$ denotes the usual set of equivalence classes of topological manifold structures on $X$ ).

Proof. Our Rough Statement, already proved, gives part of this theorem, i.e., if $\operatorname{IT}^{k}(X)$ is non-void, at least one TOP structure must exist.

We sharpen this statement by showing how an element of $\operatorname{IT}^{k}(X)$ determines precisely a single element in $\mathscr{S}_{\text {TOP }}(X)$. Naturally, the $p$-structure on $X$ which comes from this manifold structure, as in Remark 1.26 above, will be seen to represent the original intrinsic transversality structure.

Therefore, let $[\alpha] \in \mathrm{IT}^{k}(X)$ be represented by the pT map $\alpha: X^{n} \rightarrow$ $W^{n+k}$. We have seen, in the proof of the Rough Statement that we can find an $\mathrm{rT}$ map $f:(W \cup c \partial W) \times I \rightarrow T(\nu)$, realizing the natural homo- 
topy identification of $W \cup c \partial W$ with $T(\nu)$, so that $f$ is simplex-wise topologically transverse (in the sense of [17]) on one end (say, $f_{1}$ on $(W \cup c \partial W) \times\{1\})$ and, on the other, is the natural PT map $f_{0}$ directly constructed from the pT embedding $\alpha$. The $n+1$-dimensional Q.A.P.C. $\tilde{\Phi}(f)$, over $\mathbf{Z}\left[\pi_{1} X\right]$ which exists by virtue of 1.21 has boundary $\partial \tilde{\Phi}(f)=$ $\tilde{\Phi}\left(f_{0}\right) \cup \tilde{\Phi}\left(f_{1}\right)=\tilde{\Phi}\left(f_{1}\right)$ since by $1.22, \tilde{\Phi}\left(f_{0}\right)$ is the trivial Q.A.P.C. arising from the identity map $X^{n} \rightarrow X^{n}$. $\tilde{\Phi}\left(f_{1}\right)$, on the other hand, is the kernel complex of the topological surgery problem $M^{n} \rightarrow X^{n}$, where $M^{n}=$ $f_{1}^{-1}\left(X^{n}\right)$.

Now, we may, in fact, do surgery on $M^{n}$ in a specific way, i.e., we may do surgery, not merely to produce a simple homotopy equivalence, but so as to control the trace of the surgery in the following sense: Let $N^{n+1}$ be the trace of the surgery i.e., $N^{n+1}$ is an $(n+1)$-dimensional topological manifold with boundary $M^{n} \cup M_{0}^{n}$ and normal map $g$ : $\left(N^{n+1} ; M^{h}, M_{0}^{n}\right) \rightarrow(X \times I ; X \times\{0\}, X \times\{1\})$ extending $f_{1}: M^{n} \rightarrow X$, so that $g_{0}: g \mid M_{0}^{n} \rightarrow X$ is a simple homotopy equivalence. Now it is clear that such a normal map $g$ produces a Q.A.P.C. $\tilde{\Phi}(g)$ of dimension $n+1$ over $\mathbf{Z} \pi_{1} X$, with $\partial \tilde{\Phi}(g)=\tilde{\Phi}\left(g_{0} \cup-f_{1}\right)=-\tilde{\Phi}\left(f_{1}\right)$ (since $g_{0}$ is a simple homotopy equivalence). (Here $-f_{1}$ is the underlying map $f_{1}$ with reversed orientation $M^{n}, X$.)

1.28. LEMMA. The trace $N^{n+1}, g$ may be chosen (i.e., the surgery on $f_{1}$ : $M \rightarrow X$ may be performed $)$ so that $\tilde{\Phi}(g)$ realizes any abstract Q.A.P.C. $A$ over $\mathbf{Z}\left[\pi_{1} X\right]$ such that $\partial A=\tilde{\Phi}\left(f_{1}\right)$. In particular the trace may be chosen so that $\tilde{\Phi}(g)=\tilde{\Phi}(f)$.

An immediate consequence of 1.28 is the following picture. We change scale so that $g$ is now regarded as a map $g: N^{n+1} \rightarrow X \times\left[\frac{1}{2}, 1\right]$ with $g^{-1}\left(\frac{1}{2}\right)=M^{n}, g^{-1}(0)=M_{0}^{n}$. Moreover, it is easily seen that we may actually embed $N^{n+1}$ in $W \times\left[\frac{1}{2}, 1\right]$ with $N\left(W \times \frac{1}{2}\right)=M, N \cap(W \times 1)$ $=M_{0}$. (That is, we may consider the surgery of $M$ to $M_{0}$ through $N$ as an ambient surgery on a submanifold of $W$.) Thus we may extend $g$ to a map $\hat{g}:(W \cup c \partial W) \times\left[\frac{1}{2}, 1\right] \rightarrow T(\nu)$. As for $f$, we change scale and orientation, so that $f$ is now to be regarded as a map on $(W \cup c \partial W) \times$ $\left[0, \frac{1}{2}\right]$ with $f^{-1}(X) \cap[(W \cup c \partial W) \times 0]=X, f^{-1}(X) \cap\left[(W \cup c \partial W) \times \frac{1}{2}\right]$ $=M$. In this view of things, we note that $\tilde{\Phi}(\hat{g})$ is well-defined and is the same as $\tilde{\Phi}(g)$. Moreover, we may define $h=f \cup \hat{g}:(W \cup c \partial W) \times I \rightarrow$ $T(\nu)$ and have $\tilde{\Phi}(h)$ well defined; In particular $\tilde{\Phi}(h)$ is merely $\tilde{\Phi}(f) \cup$ $\tilde{\Phi}(g)$ mod identifications and may be identified with the "double" of $\tilde{\Phi}(f)=\tilde{\Phi}(f) \cup_{\partial}(-\tilde{\Phi}(f))=\partial \tilde{\Phi}(f) \times I$. [We remind the reader that 
these constructions i.e., the double $A \cup_{\partial}-A$ of Q.A.P.C.-with-boundary over $\mathbf{Z}\left[\pi_{1} X\right]$ and the "suspension" $A \times I$ may be defined purely algebraically, and, of course $\partial(A \times I)=A \cup \cup_{\partial}-A$.]

It is clear now that we wish to define the map $\operatorname{IT}^{k}(X) \rightarrow \mathscr{S}_{\text {TOP }}(X)$ via the assignment $[\alpha] \rightarrow\left(g_{0}: M_{0} \rightarrow X\right)$, where $M_{0}, g_{0}$ are constructed precisely as above.

It now remains to show that this construction has the standard properties i.e., that it is well-defined, and that it, in fact, induces a bijection of sets.

The first of these properties must be established in two stages. We must show first of all that for a given $\alpha$ we obtain a unique $N_{0}, g_{0}$ up to equivalence within the structure set $\mathscr{S}_{\text {TOP }}(X)$. Then it must be demonstrated that no indeterminacy is introduced by varying the choices of the representative $\alpha$.

Let us examine then the ambiguity which occurs in the construction once $\alpha$ is fixed. Basically, this involves the choice of $f$, and the trace. Recall that $f$ is a map $(W \cup c \partial W) \times I \rightarrow T(\nu)$ which is RT on each simplex of the homotopy cylinder, and which is the PT map $f_{0}$ (determined by $\alpha$ ) on one end and simplex-wise topologically transverse on the other.

First, fix $f$ and let us examine how the choice of $N$ affects things. Let $N^{a}, g^{a}, N^{b}, g^{b}$ indicate two such choices. If $f$ is indicated schematically thus

$$
\begin{array}{ll}
f_{0} f & f \\
f_{1}
\end{array}
$$

then the two choices of $N, g$ may be indicated thus

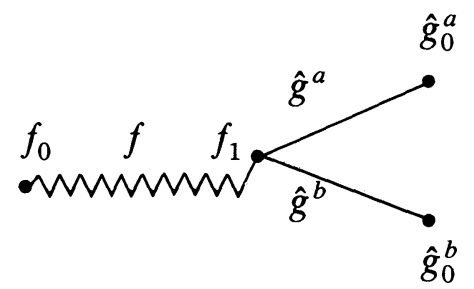

Here, the circumflex denotes the map on $(W \cup c \partial W) \times$ interval dictated by the actual normal bordism. Wavy lines indicate simplex-wise "RT"maps and straight lines topologically transverse maps. If we allow a minus sign to indicate a change of orientations, we obtain a topologically transverse homotopy

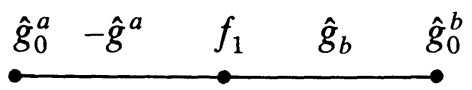


which produces a topological normal bordism $-g^{a} \cup_{f_{1}} g^{b}$ between $g_{0}^{a}$ and $g_{0}^{b}$. It is clear from the construction that $\tilde{\Phi}\left(-\hat{g}^{a} \cup \hat{g}^{b}\right)=$ double of $\tilde{\Phi}(f)$. But the clear interpretation of this is that the two structures $g_{0}^{a}: M_{0}^{a} \rightarrow X$, $g_{0}^{b}: M_{0}^{b} \rightarrow X$ obtained via $g^{a}, g^{b}$ respectively differ by the action of an element of $L_{n+1}\left(\pi_{1} X\right)$ i.e. they are normally cobordant. However, this element is represented by $\tilde{\Phi}\left(-g^{a} \cup g^{b}\right)$ and since the latter, being a double, is clearly bordant to zero, it must represent the trivial element. Thus by standard surgery theory the two structures are equivalent. Hence we have shown that, give $f$, the particular choice of $N$ and $g$ is immaterial.

Now let $f^{a}, f^{b}$ be two choices of the homotopy $f$, and $M_{1} M_{1}^{a}, M_{1}^{b}$, $f_{1}^{a}, f_{1}^{b}$ etc. the resulting manifolds and maps per the construction outlined above.

Step 1. Suppose $f^{a}$ differs from $f^{b}$ in the following respect: $f^{b}$ is obtained by taking $f^{a}$ and concatenating it with a further homotopy $f^{x}$ from $f_{1}^{a}$ to $f_{1}^{b}$ so that $f^{x}$ is simplex-wise topologically transverse. Schematically, we indicate things thus:

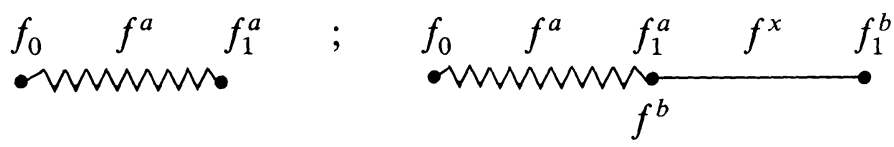

Here, as before, wavy lines stand for the part of the homotopy which is merely RT and straight lines for topologically transverse maps. The choice $f^{a}$ produces a trace $N^{a}$ connecting $f_{1}^{a}$ to a homotopy $g_{0}^{a}$ equivalence via $g^{a}$, and we obtain, schematically

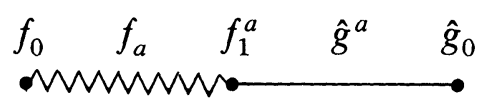

It is clear, however, that $N^{b}, g^{b}$ can be chosen as the concatenation of $g^{a}$ with $-\iota^{x}$ where $g^{x}=f^{x} \mid\left(f^{x}\right)^{-1} X$ is the obvious topological normal bordism between $M_{1}^{a}, f_{1}^{a}$ and $M_{1}^{b}, f_{2}^{b}$. Thus, schematically

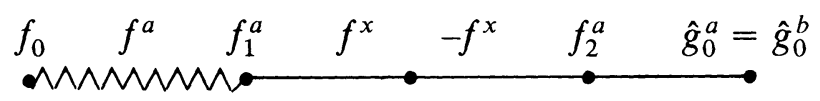

i.e. $M_{0}^{a}, g_{0}^{a}=M_{0}^{b}, g_{0}^{b}$.

Step 2. Now let $f^{a}, f^{b}$ be arbitrary. First observe that, using ordinary topological transversality (via [17]) it is clear that $f_{1}^{a}$ is homotopic to $f_{1}^{b}$ via $f^{g}$ where $f^{g}$ is topologically transverse. Therefore, in view of Step 1 above, we may as well assume that $f_{1}^{a}=f_{1}^{b}$. Thus schematically, the 
construction of the two possible structures is indicated thus

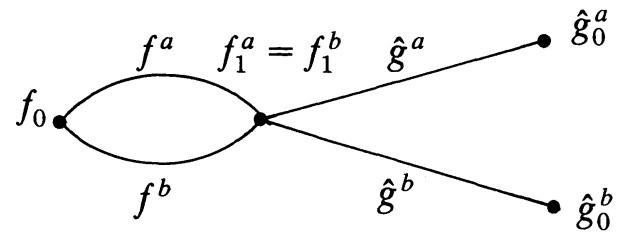

The following diagram illustrates a normal bordism $h$ between two topological manifold structures $g_{0}^{a}: M_{0}^{a} \rightarrow X, g_{0}^{b}: M_{0}^{b} \rightarrow X$ on $X$ :

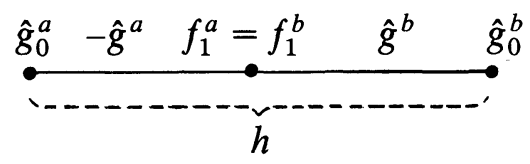

The closed Q.A.P.C. $\hat{\Phi}(\hat{h})$ over $\mathbf{Z}\left[\pi_{1} X\right]$ determines an element of $L_{n+1}\left(\pi_{1} X\right)$ measuring the difference between the two topological manifold structures $g_{0}^{a}: M_{0}^{a} \rightarrow X, g_{0}^{b}: M_{0}^{b} \rightarrow X$. The problem, then is to show that this element is inertial, i.e., $[\tilde{\Phi}(h)]$ is in the image of $\left[\sum X, G /\right.$ TOP]. We note, on the algebraic level that $\tilde{\Phi}(h)=-\left(\tilde{\Phi}\left(f^{a}\right) \cup_{\partial}-\tilde{\Phi}\left(f^{b}\right)\right)$ by construction of $g^{a}, g^{b}$. It follows that if we can show $\left(\tilde{\Phi}\left(f^{a}\right) \cup_{\partial}-\tilde{\Phi}\left(f^{b}\right)\right)$ represents an inertial element in $L_{n+1}\left(\pi_{1} X\right)$ we will have completed the necessary verification. There are several ways of seeing this of which the following is an example.

Consider the homotopy $e$ of $\hat{g}_{0}^{a}$ to itself schematically described by the following diagram

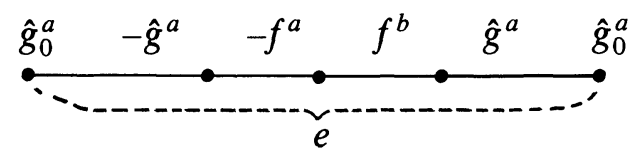

It is clear that $\tilde{\Phi}(e)$ is defined as a closed $(n+1)$-dimensional Q.A.P.C. and may be evaluated by the formula

$$
\tilde{\Phi}(e)=\left(-\tilde{\Phi}\left(\hat{g}^{a}\right) \cup_{\partial} \tilde{\Phi}\left(f^{a}\right)\right) \cup\left(\tilde{\Phi}\left(g^{a}\right) \cup_{\partial} \tilde{\Phi}\left(f^{b}\right)\right)
$$

but we claim that the right side of this equation is clearly bordant, in the world of Q.A.P.C.'s to $-\Phi\left(f^{a}\right) \cup_{\partial} \Phi\left(f^{b}\right)$. [This is perfectly analogous (and, given the technical preliminaries as perfectly straightforward) as the following obvious fact about oriented manifolds: Given manifolds $A, B, C$ with boundary and $\partial A=-\partial B=\partial C$ then $\left(A \cup \cup_{\partial}-C\right) \cup\left(B \cup_{\partial} C\right)$ is bordant to $\left(A \cup{ }_{\partial} B\right) \cup\left(C \cup_{\partial}-C\right)$ which in turn is bordant to $A \cup{ }_{\partial} B$ (since $C \cup_{\partial}-C$ is a double).] Therefore, we need only show that $[\tilde{\Phi}(e)]$ is inertial. Note, however, that the trace $N^{a}$ of the surgery form $M^{a}=M^{b}$ 
to $M_{0}^{a}$ may be viewed as a submanifold of a copy of $(W \cup c \partial W) \times I$ away from the cone point and in general position with respect to a triangulation ( $M^{a}$ is already in general position with respect to the triangulation of the copy of $W \cup c \partial W$ in which it lies). This being so, we may derive from the simplex-wise topologically transverse map $\hat{g}_{0}^{a}: W \cup$ $c \partial W \rightarrow T(\nu)$ a section of $s: \mathscr{Y}(\nu) \rightarrow T(\nu)$ and from the self-homotopy $e$ (which is simplex-wise at least RT) a self-homotopy $S$ of $s_{0}$ in the world of sections. An immediate corollary of 1.11 reveals that if a section, e.g., $s_{0}$ arises from explicitly using a topological stucture on $\nu$ and Scharlemann topological transversality then a self-homotopy of the section is homotopic (through self-homotopy's of the same fixed section) to a self-homotopy which is (simplex-wise) topologically transverse. [Alternatively, we may present this fact by saying that homotopy classes of self-homotopies of $s_{0}$ are in 1-1 correspondence with homotopy classes of topological self-equivalences $\nu \rightarrow \nu$ which are fiber homotopically the identity.]

In consequence, we have the following diagram representing a homotopy $E$ of $e \operatorname{rel}(W \cup c \partial W) \times I$ to $e^{\prime}$

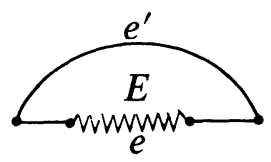

Here $e^{\prime}$ is simplex-wise topologically transverse while $E$ is RT. It follows that $\left[\tilde{\Phi}\left(e^{\prime}\right)\right]=[\tilde{\Phi}(e)]$ but $\tilde{\Phi}\left(e^{\prime}\right)$ is the surgery obstruction of the normal bordism $d \times 1_{I}: N_{d} ; M_{0}^{a}, \quad M_{0}^{a} \rightarrow X \times I, X \times 0, X \times 1$ where $N_{d}=$ $\left(e^{\prime}\right)^{-1} X$ and $d=e^{\prime} \mid N_{e}$. Thus, clearly, $\Phi\left(e^{\prime}\right)$ is inertial. (Alternatively, one can argue that $\left[\Phi\left(e^{\prime}\right)\right]$ arises from a topological self-equivalence $\nu \rightarrow \nu$ and thus, by definition, is in the image of [ $\left.\sum X, G / \mathrm{TOP}\right]$.) This completes Step 2, and we have thus far shown that, for a given $p$-structure $\alpha$, there is a well-defined topological manifold structure on $X$. It remains to show that altering $\alpha$ within its equivalence class in $\operatorname{IT}^{k}(X)$ does not affect the equivalence class of the induced manifold structure on $X$. Therefore, let $\alpha^{x}, \alpha^{y}$ be two equivalent $p$-structures on $X$ and $f_{0}^{x}, f_{0}^{x}$ the two simplexwise PT maps $W \cup c \partial W \rightarrow T(\nu)$ arising from $\alpha^{a}, \alpha^{b}$ via the usual construction. Now, the topological manifold structure $g_{0}^{x}: M_{0}^{x} \rightarrow X$ arising from $\alpha^{x}$ is obtained, schematically thus

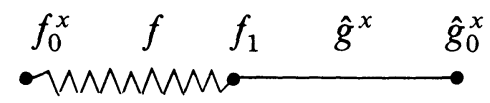

where $g_{0}^{x}: M_{0}^{x} \rightarrow X$ is a simple homotopy equivalence, and $g_{x}: N_{x} \rightarrow$ $X \times I$ a normal bordism. It is immediate from the definition of equiva- 
lence among $p$-structures that there is a homotopy $F$ from $f_{0}^{x}$ to $f_{0}^{y}$, simplex-wise $\mathrm{RT}$, such that $[\hat{\Phi}(F)] \in \operatorname{im}\left[\sum X, G / \mathrm{TOP}\right] \subseteq L_{n+1}\left(\pi_{1} X\right)$. Therefore, by the preceding arguments, we may describe the topological manifold structure induced by $\alpha^{y}$ as having been constructed as follows:

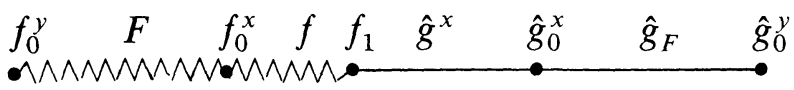

Here the concatenation of $f$ and $F$ is the homotopy we choose to deform $f_{0}^{b}$ through an RT map to a topologically-transverse map. Moreover, $\hat{g}_{F}$ arises from a normal bordism $g_{F}: N_{F} \rightarrow X \times I$ where $N_{F}$ is the trace of a topological surgery chosen so that the surgery obstruction Q.A.P.C. of $g_{F}$, i.e. $\hat{\Phi}\left(\hat{g}_{F}\right)$ is $-\tilde{\Phi}(F)$. Clearly, $\tilde{\Phi}\left(\hat{g}^{x} \cup \hat{g}_{F}\right)=-\tilde{\Phi}(F \cup f)$, so that the choice of $N_{F}, g_{F}$ (which converts the simple homotopy equivalence $g_{0}^{x}$ : $M_{0}^{x} \rightarrow X$ to another simple homotopy equivalence $\left.g_{0}^{y}: M_{0}^{y} \rightarrow X\right)$ to be concatenated with $N^{x}, g^{x}$ yields a trace $N^{x} \cup N^{F}$ which meets the requirements of the original construction. But then $M_{0}^{x}, g_{0}^{x}$ and $M_{0}^{y}, g_{0}^{y}$ are normally bordant through a bordism whose surgery obstruction is - $[\tilde{\Phi}(F)]$ and is thus in $\operatorname{im}\left[\sum X, G / \mathrm{TOP}\right]$, hence inertial, so $M_{0}^{x}, g_{0}^{x}$ and $M_{0}^{y}, g_{0}^{y}$ are equivalent manifold structures on $X$.

So we may finally conclude that $\operatorname{IT}^{k}(X) \rightarrow \mathscr{S}_{\text {TOP }}(X)$ has been well defined.

We have already described the inverse map $\mathscr{S}_{\mathrm{TOP}}(X) \rightarrow \mathrm{IT}^{k}(X)$ and it is the work of a moment to see that it is in fact an equivalence. Thus 1.27 is proved.

We now turn to the question of putting a topological manifold structure on a $P$-pair, i.e., given a $P$-pair $\left(X^{n}, \partial X^{n}\right)(n \geq 6 \partial X \neq 0)$, we investigate conditions equivalent to the existence of a manifold-withboundary $M^{n}$ and a simple homotopy equivalence of pairs $f: M^{n}$, $\partial M^{n} \rightarrow X^{n}, \partial X^{n}$. The results are analogous to those for $P$-spaces, i.e., 1.27 .

Given $\left(X^{n}, \partial X^{n}\right)$ consider a relative regular neighborhood $\left(W^{n+k}, V^{n+k-1}\right)$, i.e., $W^{n+k}$ is a regular neighborhood of $X^{n}$ in $\mathbf{R}^{n+k}$; moreover, $V^{n+k-1}$ is a codimension-0 submanifold of $\partial W$, and there is a simple homotopy equivalence $S: X^{n}, \partial X^{n} \rightarrow W^{n+k}, V^{n+k-1}$. Hereafter, when we speak of $\left(W^{n+k}, V^{n+k-1}\right)$ as a "regular neighborhood" of $X^{n}$, $\partial X^{n}$ we shall implicitly have in mind a particular simple homotopy equivalence $S$ (well-defined up to homotopy).

First, we extend some definitions slightly. Let $Y^{n+k}$ be a combinatorially triangulated manifold and $Z^{n+k-1}$ a codimension- 0 submanifold of $\partial Y$ which is a subcomplex of the given triangulation. Let $\left(X^{n}, \partial X^{n}\right)$ be a $P$-pair; $f:(X, \partial X) \rightarrow(Y, Z)$ is said to be pT iff $f^{-1}(Z)=\partial X$, 
$f^{-1}(\partial Y-Z)=\varnothing$, and $\left(f^{-1} \sigma, f^{-1} \dot{\sigma}\right)$ is a $P$-pair of dimension $j-k$ for each $j$-dimensional simplex $\sigma$ of $Y$.

1.28. Definition. A $p$-structure $\left(X^{n}, \partial X^{n}\right)$ consists of a regular neighborhood $\left(W^{n+k}, V^{n+k-1}\right)$ of $(X, \partial X)$ in $\mathbf{R}^{n+k}$ (with $S:(X, \partial X)^{\sim} \rightarrow$ $(W, V))$, a $P$-pair $\left(X_{1}^{n}, \partial X_{1}^{n}\right)$, a triangulation of $W$ with $V$ as a subcomplex, a simple homotopy equivalence of pairs $h:(X, \partial X) \rightarrow\left(X_{1}, \partial X_{1}\right)$ and a pT map $g:\left(X_{1}, \partial X_{1}\right) \rightarrow(W, V)$ such that

$$
\begin{gathered}
(X, \partial X) \stackrel{\sim}{\rightarrow}(W, V) \\
\downarrow h \quad \nearrow g \\
\left(X_{1}, \partial X_{1}\right)
\end{gathered}
$$

is homotopy commutative.

We shall defer for a while the definition of a suitable equivalence relation among $p$-structures. We shall, however, note that the "Rough Statement" approximating Theorem 1.27, carries over well enough to the case of a $P$-pair i.e.

1.29. TheOrem. A P-pair $\left(X^{n}, \partial X^{n}\right)(n \geq 6)$ has the simple homotopy type of a topological manifold, mod its boundary, if and only if $(X, \partial X)$ admits a p-structure (for one codimension $k>n$ ). In fact, a p-structure determines a particular topological structure (up to concordance).

Proof. That a topological manifold with boundary admits a $p$-structure is as obvious as in the case of a closed manifold.

The proof of the converse is modeled, to a great extent, on that of 1.27 , as one would expect. However, some additional subtlety is needed. As in 1.27 , we may as well assume that there is a pT-map $f:(X, \partial W) \rightarrow$ $(W, V)$ with respect to some triangulation of $W$, and that $f$ is an embedding. As before, we obtain a specific topological reduction of $\nu(X, \partial X)$, since the $\mathrm{pT}$ map $(X, \partial X) \rightarrow(V, \partial V)$ leads to a specific transversality structure on $\nu$. The problem now, is to do surgery of the following sort: we shall have a manifold $\left(M^{n}, \partial M^{n}\right)$ which admits a degree one normal map to $(X, \partial X)$ and we shall want to perform surgery on $\partial M \rightarrow \partial X$ to make it into a homotopy equivalence; this will leave us with a degree one normal map $M, \partial M \rightarrow X, \partial X$ already a simple homotopy equivalence on the boundary, and we shall need to see that surgery in int $M$ may be done to make this a simple homotopy equivalence. Finally, we shall observe our procedure produces an essentially unique manifold structure on $(X, \partial X)$. 
The first point essentially replicates the argument of 1.27 . That is, using the given pT structure on $(X, \partial X)$, we obtain one on $\partial X$ by restriction, and hence a manifold structure on $\partial X$. More specifically, the pT structure $\alpha$ on $(X, \partial X)$ determines a specific homotopy equivalence (homotopic to the standard one).

$$
f_{0}:(W \cup c \bar{\partial} W, V \cup c \partial V) \rightarrow\left(T(\nu), T\left(\nu_{0}\right)\right) .
$$

Here $\bar{\partial} W=\operatorname{cl}(\partial W-V)$, and $\nu_{0}=\nu \mid \partial X . f_{0}$ is, of course, simplex-wise PT. Now, in the spirit of 1.27 , we deform $f_{0}$, via $f$, a simplex-wise RT map to $f_{1}$, a simplex-wise topologically transverse map. Schematically

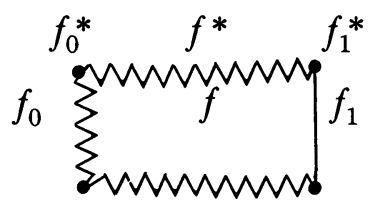

Here, the notation ${ }^{*}$ in superscript denotes restriction to $V \cup c \partial V$. Let $M=f_{1}^{-1} X$ with $\partial M=f_{1}^{-1} \partial X$. Again, wavy lines connote RT, straight topological transversality. We do surgery on $\partial M, f^{*} \mid \partial M$ via $N^{*}, g^{*}$ as in the proof of 1.27 to produce

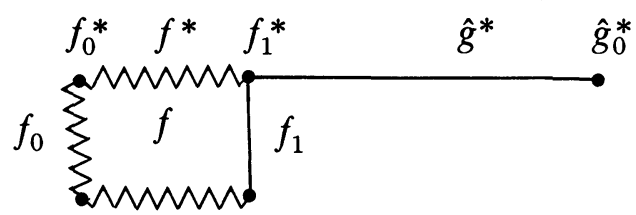

where $g_{0}^{*}: M_{0}^{*} \rightarrow \partial X$ is a simple homotopy equivalence. We then do surgery on $M \cup N^{*}$ rel $M_{0}^{*}$ to produce the following situation

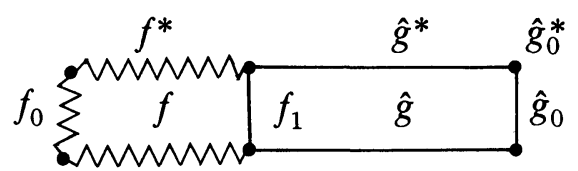

where $g$ has trace $N$ with boundary $\left(M \cup N^{*}\right) \cup_{M_{0}^{*}} M_{0}, \partial M_{0}=M_{0}^{*}$ and moreover, $g_{0}$ is a $M_{0}^{*}$ simple homotopy equivalence. The algebraic requirement which we insist on in characterizing $N$ and $g$ is the following. If $\tilde{\Phi}\left(f^{*}\right), \tilde{\Phi}\left(g^{*}\right)$ etc. are Q.A.P.C.'s over $\mathbf{Z}\left[\pi_{1} \partial X\right]$, let $\tilde{\Phi}_{\pi}\left(f^{*}\right), \tilde{\Phi}_{\pi}\left(g^{*}\right)$ etc. denote their respective images under the functor from Q.A.P.C.'s over $\mathbf{Z}\left[\pi_{1} \partial X\right]$ to those over $\mathbf{Z}\left[\pi_{1} X\right]$ induced by the obvious homomorphism $\pi_{1} \partial X \rightarrow \pi_{1} X$. We then have two triads of Q.A.P.C.'s over $\mathbf{Z}\left[\pi_{1} X\right]$.

(i) $\left(\tilde{\Phi}(f) ; \tilde{\Phi}\left(f_{1}\right) ; \tilde{\Phi}_{\pi}\left(f^{*}\right) ; \tilde{\Phi}_{\pi}\left(f_{1}^{*}\right)\right)$ and

(ii) $\left(\tilde{\Phi}(g),-\tilde{\Phi}\left(f_{1}\right), \tilde{\Phi}_{p} i\left(g^{*}\right),-\Phi_{\pi}\left(f_{1}^{*}\right)\right)$

Here $\partial \tilde{\Phi}(f)=\tilde{\Phi}\left(f_{1}\right) \bigcup_{\partial} \tilde{\Phi}_{\pi}\left(f^{*}\right)$ where the union is along the common boundary $\partial \tilde{\Phi}\left(f_{1}\right)=-\partial \tilde{\Phi}_{\pi}\left(f^{*}\right)=\tilde{\Phi}_{\pi}\left(f_{1}^{*}\right)$. A like formula holds for the second triad. 
Our requirement on the surgery then is that (ii) $=-(\mathrm{i})$, i.e., the second triad is isomorphic to the first with change of orientation, the isomorphsm preserving the identity on $-\tilde{\Phi}\left(f_{1}\right)$.

We claim that the surgery may be done so that this will hold.

This completes the proof of 1.29 as stated.

As an addendum we sharpen 1.29 slightly by specifying the correct notion of equivalence among $p$-structures. Let $G, H$ be groups and $\phi$ : $G \rightarrow H$ a fixed homomorphism. Consider triples $(A, B, h)$ where $A$ is an $n$-dimensional Q.A.P.C.-with-boundary over $\mathbf{Z}[H], \quad B$ is an $(n-1)$ dimensional closed Q.A.P.C. over $\mathbf{Z}[G]$, and $h$ is a particular equivalence of $\phi_{\#} B$ with $\partial A$, where $\phi_{\#}$ denotes the function from Q.A.P.C.'s over $\mathbf{Z}[G]$ to Q.A.P.C.'s over $\mathbf{Z}[H]$ induced by $\phi$. It is possible to define bordism between two such objects straightforwardly, and we denote the group of bordism classes by $L_{n}(\phi: G \rightarrow H)$ or, more briefly, $L_{n}(\phi)[16$, $\S 2.2]$.

Let $(X, \partial X)$ be a $P$-pair and let $\phi$ denote the homomorphism $\pi_{1} \partial X \rightarrow \pi_{1} X$ induced by inclusions. Let $e, e^{\prime}: X, \partial X \rightarrow W, V$ be two $p$-structures on $X, \partial X$. Just as in the closed case we have maps $\hat{e}, \hat{e}_{0}^{\prime}$ : $(W \cup c \bar{\partial} W, V \cup c \partial V) \rightarrow\left(T(\nu), T\left(\nu_{\partial}\right)\right)$ which are PT simplex-wise.

1.30. Definition. $\alpha$ and $\beta$ are said to be equivalent if and only if there is an RT homotopy $\hat{f}:(W \cup c \bar{\partial} W, V \cup c \partial V) \times I \rightarrow\left(T(\nu), T\left(\nu_{\partial}\right)\right)$ connecting $\hat{e}_{\alpha}, \hat{e}_{\beta}^{\prime}$ so that $[\tilde{\Phi}(\hat{f})] \in L_{n}(\phi)$ is the trivial element.

1.31. Definition. $\operatorname{IT}^{k}(X, \partial X)$ is the set of equivalence classes of $p$-structures on $(X, \partial X)$.

1.32. Corollary. For $n \geq 6$ (or $n \geq 5$ if $\partial X=\varnothing$ ). There is a natural bijection $\operatorname{IT}^{k}(X, \partial X) \rightarrow \mathscr{S}_{\mathrm{TOP}}(X, \partial X)$.

1.31. Remark. In comparing Definition 1.30 with Definition 1.24 , it will be noted that there is a discrepancy between the two in that, in the case of a $P$-space, $X$, i.e. $\partial X=\varnothing, 1.24$ and 1.30 give definitions of equivalence of $p$-structures which are not clearly equivalent a priori. That is, if we apply 1.30 to a closed $P$-space $X$, we find that it specifies that $[\hat{\Phi}(\hat{f})]=0$ in $L_{n}\left(\pi_{1} X\right)$, whereas 1.24 merely asserts that $[\tilde{\Phi}(\hat{f})] \in$ $\operatorname{im}\left[\sum X, G / \mathrm{TOP}\right]$. However, it is easily seen that the proof of 1.27 goes through virtually without modification if the stronger equivalence relation be substituted for the weaker in the definition of $\operatorname{IT}^{k}(X)$. This of itself 
shows that the two notions of equivalence are the same (at least for $\operatorname{dim} X>5$ ). In fact, without reference to 1.27 or the dimension of $X$ it is possible to show directly that the weaker equivalence implies the stronger. Thus the apparent disparity disappears.

Finally, we remark that as before, there is a natural suspension $\operatorname{IT}^{k}(X, \partial X) \rightarrow \operatorname{IT}^{k+1}(X, \partial X)$ which is bijective for large $k$ hence we may speak of stable intrinsic transversality structures, i.e. IT $(X, \partial X)$

\section{Applications.}

2.1. Manifolds as covering spaces. In this application we consider the following problem: Let $M^{n}$ be a closed manifold having the simple homotopy type of a (finite) covering space. Under what conditions is it then the case that $M^{n}$ itself is a covering space?

Throughout the following section assume that $M^{n} \simeq X$ (simply) and that $p: \tilde{X} \rightarrow X$ is a covering finite map, with $X$ a finite CW complex. It is easily seen that, under such an assumption $X$ is a PD space of formal dimension $n$. For the sake of simplicity, we shall further assume that $\tilde{X}$ is orientable (hence $M^{n}$ is orientable as a manifold).

Moreover, we may make the assumption that $X$ is in fact a high-dimensional parallelizable manifold - with boundary (i.e., as a manifold $X$ has dimension $n+k, k>n)$.

This follows since we may replace $X$ by its regular neighborhood in $\mathbf{R}^{n+k}$. If $X$ is such a manifold, then $\tilde{X}$ is of course a manifold as well.

With these assumptions understood, the problem, then, is to find a manifold $V^{n}$, simply homotopy equivalent to $X$, so that $M^{n}$ is the covering of $V^{n}$ induced by the homotopy equivalence. We shall state a sufficient condition that this can be done which is, almost trivially, a necessary condition.

Suppose that $M^{n}$ does, in fact, cover $V^{n}$ so that $M^{n} \stackrel{p^{\prime}}{\rightarrow} V^{n}$ is, homotopically, $p: \tilde{X} \rightarrow X$.

We may then embed $V^{n}$ in $X$ (since $X$ is a high-dimensional manifold) via a simple homotopy so that $p^{-1} V^{n}=M^{n} \subset \tilde{X}$.

Suppose $X$ is combinatorially triangulated (which may be done since $X$ is a codimension zero submanifold of Euclidean space).

We may assume that $V^{n} \subset X$ is in general position with respect to the triangulation. Therefore, $M^{n} \subset \tilde{X}$ is in general position with respect to the triangulation of $\tilde{X}$ induced by $p$ from that on $X$.

Moreover, $V^{n}$ obviously has a small regular neighborhood $U$ in $X$ (i.e., a smaller copy of $X$ ) so that $U \cap \sigma$ is a regular neighborhood of $V \cap \sigma$ for every simplex $\sigma$ of $X$. Therefore $p^{-1} U=\tilde{U}$ is a regular 
neighborhood of $M^{n}$ having the same property, viz. $\tilde{U} \cap \sigma$ is a regular neighborhood of $M \cap \sigma$ for every simplex $\sigma$ of $\tilde{X}$.

We wish to prove the converse of the remark above when $n \geq 5$. Let $M^{n}, X, \tilde{X}, p$ be as above.

2.1.1. Suppose $M^{n}$ can be embedded (via a simple homotopy equivalence) in $\tilde{X}$, in topological general position with respect to the triangulation, so that $M$ has a regular neighborhood $\tilde{U}$ satisfying

(1) $\tilde{U} \cap \sigma$ is a regular neighborhood of $M^{n} \cap \sigma$.

(2) $\tilde{U}$ is the covering space $p^{-1}(p \tilde{U})$.

Then there is a manifold $V^{n}$ and a simple homotopy equivalence $\phi$ : $V^{n} \rightarrow X$ so that the covering space of $V$ induced by $\phi$ is homeomorphic to $M^{n}$. Moreover, this homeomorphism is consistent with the original simple homotopy equivalence $M^{n} \rightarrow \tilde{X}$.

Before proving this, let us observe what the theorem is saying: if $M^{n}$ could be embedded in $\tilde{X}$ so as to cover its image under $p$, finding $V^{n}$ would be vacuously simple. What the theorem says is that if $M^{n}$ be replaced by an equivalent PD space (i.e. $\tilde{U}$ ) and the embedding $M^{n} \rightarrow \tilde{X}$ by a pT embedding of $\tilde{U}$ "homotopy equivalent" to the original, (in the sense that $(\tilde{U} \cap \sigma) \sim\left(M^{n} \cap \sigma\right)$ so that $\tilde{U}$ covers its image under $\left.p\right)$, then we can arrange to have $M^{n}$ itself cover its image under $p$.

The proof is a straightforward application of the results of $\S 1$. Let $U=p \tilde{U}$. Obviously $U$ is an orientable PD space of dimension $n$. Clearly, $U \subset X$ is a pT embedding, since, for each $\sigma^{j+k}, U \cap \sigma^{j+k} \cong \tilde{U} \cap \bar{\sigma}^{j+k}$ where $\bar{\sigma}^{j+k}$ is a component of $p^{-1} \sigma^{j+k}$. But $\tilde{U} \cap \bar{\sigma} \sim M^{n} \cap \bar{\sigma}$ which, since $M^{n}$ is in general position with respect to the triangulation, is at least a homology manifold with boundary $M^{n} \cap \partial \bar{\sigma} \simeq U \cap \partial \sigma$.

Thus, we may assert that $U$ admits the structure of a topological manifold $V^{n}$, by virtue of 1.27 . In fact, we may assert that, if we embed $V^{n}$ in $X$ in general position with respect to the triangulation then there is a normal space $N^{n+1}$ (with normal fibration induced from $\nu(X)$, i.e. $\nu(V)$ ), rT embedded in $X \times I$, so that $\partial N^{n+1}=V^{n} \cup U$, with $V^{n} \subset X \times$ $\{0\}, U \subset X \times\{1\}$ the original embeddings, and such that the surgery problem $\left(N^{n+1} ; V^{n}, U\right) \rightarrow(X \times I ; X \times\{0\}, X \times\{1\})$ has vanishing obstruction in $L_{n+1}\left(\pi_{1} X\right)$. We now look at the induced cover $p \times$ id $=q$ : $\tilde{X} \times I \rightarrow X \times I$, and at $q^{-1}\left(N^{n+1}\right)=\tilde{N}^{n+1}$, which is an rT-embedded normal space connecting $U$ and $M^{\prime}=q^{-1} V^{n}$, where $M^{\prime}$ is embedded in $\tilde{X} \times\{0\}$ topologically transverse to the triangulation. Obviously, this gives a surgery problem $\left(N^{n+1}, M^{\prime}, U\right) \rightarrow(X \times I, X \times\{0\}, X \times\{1\})$ which has surgery obstruction zero in $L_{n+1}\left(\pi_{1} X\right)=L_{n+1}\left(\pi_{1} M^{n}\right)$. But then the $p$-structure on the homotopy type of $M^{\prime}$ given by the general 
position embedding of $M^{\prime}$ in $\tilde{X} \times\{0\}$ is equivalent to that given by the pT embedding $\tilde{U} \subset \tilde{X} \times\{1\}$, which is clearly the same as the $p$-structure coming from the original general position embedding of $M^{n}$ in $X$. Thus the topological manifold structure of $M^{\prime}$ on the underlying simple homotopy type is the same as that of $M^{n}$, and the proof is complete.

2.1.2. Remark. Suppose the covering $\tilde{X} \rightarrow X$ comes from a free action of the finite group $G$ on $X$, i.e., $X=\tilde{X} / G$. Then the condition (2) on $U$ in the statement of Theorem 2.2.1 may be paraphrased by saying that $U$ is a $G$-invariant tubular neighborhood; moreover, the conclusion of 2.2.1 may be taken to say that $M^{n}$ admits a certain free topological $G$-action.

2.2. Decomposing a manifold as a product. In this section we ask a question similar to that of 2.1: If $M^{n}$ is a manifold having the homotopy type of $X^{n-d} \times W^{d}$, where $W^{d}$ is a (PL) manifold and $X^{n-d}$ a P.D. space, when can we find a topological manifold structure $V^{n-d}$ on $X$ so that $M^{n} \cong V^{n-d} \times W^{d}$ ?.

Again, we rephrase this slightly: Let $X^{n+k-d}$ be a parallelizable manifold (i.e., a codimension 0 submanifold of $\mathbf{R}^{n+k-d}$ ), let $Y=X \times W^{d}$, and let $X, Y$ be triangulated so that the projection $p: Y \rightarrow X$ is simplicial, i.e., triangulate $W$ and take the product triangulation on $Y$. Suppose $f$ : $M \rightarrow Y^{n+k}$ is a simple homotopy equivalence: When may we decompose $M^{n}$ appropriately as $V^{n-d} \times W^{d}$, i.e., when may we find a topological manifold $V^{n-d} \subset X$, with the inclusion a simple homotopy equivalence, so that $p^{-1} V \cong V^{n-d} \times W^{d}$ is homeomorphic to $M^{n}$ (consistent, up to homotopy with the map $f)$ ? The initial result is similar to 2.1.1. Let $n-d \geq 5$.

2.2.1. TheOREM. Suppose $f$ is an embedding $M^{n} \hookrightarrow Y^{n+k}$, and suppose that the embedding is in topological general position with respect to the triangulation of $Y$. Suppose further that the embedded $M^{n}$ has a regular neighborhood $T$ such that

(1) $T \cap \sigma$ is a regular neighobrhood of $M^{n} \cap \sigma$ for all simplices $\sigma$ of $Y$

(2) $T=p^{-1}(p T)$ i.e. $T=p(T) \times W^{d}$.

Then there is a topological submanifold $V^{n-d} \subset X^{n+k-d}$, embedded via a simple homotopy equivalence, so that $p^{-1}(V)=V \times W$ is homeomorphic to $M^{n}$.

Proof. The proof follows the general outlines of 2.1.1. Let $U=p(T)$. Since $U \times W^{d}=T \cong M^{n}, U$ must be a P.D. space of dimension $n-d$. Furthermore, let $K_{\sigma}=p^{-1} \sigma$, for each simplex $\sigma^{j}$ of $X, K_{\dot{\sigma}}=p^{-1} \dot{\sigma}$. Let $U_{\sigma}$, 
$U_{\dot{\boldsymbol{\sigma}}}$ denote $U \cap \sigma, U \cap \dot{\sigma}$. Then $\left(T \cap K_{\sigma}, T \cap K_{\dot{\sigma}}\right) \sim\left(M \cap K_{\sigma}, M \cap K_{\dot{\sigma}}\right)$ and is thus a P.D. pair. But $\left(T \cap K_{\sigma}, T \cap K_{\dot{\sigma}}\right)=U_{\sigma} \times W, U_{\dot{\sigma}} \times W$ and thus $U_{\sigma}, U_{\dot{\sigma}}$ is a P.D. pair. Thus $U \subset X$ is a pT-embedding, i.e. a $p$-structure on $U$. Since $n-d \geq 5$, by 1.27 we may find a manifold $V^{n-d}$ of the simple homotopy type of $U$. In fact, consistent with this simple homotopy equivalence, we may embed $V^{n-d}$ in $X$, topologically transverse to the triangulation so that there exists a $\nu^{k}(U)=\nu^{k}(V)$ normal space $R$, with $\partial R=U \cup V$, so that $R$ is rT embedded in $X \times I$ (with respect to some triangulation), extending the given embeddings $V^{n-d} \subset X$, $U \subset X$ on either end.

But then $(p \times d)^{-1} R \cong R \times W^{d} \cup Y \times I$ is a $\nu\left(M^{n}\right)$ normal space with boundary $\partial(R \times W)=\left(V^{n-d} \times W\right) \cup T$ embedded in $Y \times I$, extending the pT embeddings $V \times W \subset Y, T \subset Y$ on each end. But the $p$-structure on the underlying homotopy type of $T \sim M^{n}$ is essentially that which comes from embedding $M$ in general position; thus $M^{n} \cong$ $V^{n-d} \times W^{d}$, the homeomorphism realizing the obvious homotopy equivalence. This completes the proof.

More generally, we may concern ourselves with the problem of fibering $M^{n}$ by $W^{d}$, i.e. find a topological fibration

$$
\begin{array}{r}
W^{d} \rightarrow M^{n} \\
\downarrow \\
V^{n-d}
\end{array}
$$

given that the homotopy type of $M^{n}$ is realized by the total space of some such fibration. Unfortunately, it is rather difficult to see what the precise statement should be; that is, assume that there is a fibering

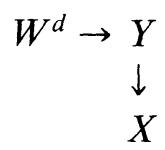

where $Y$ has the simple homotopy type of $M^{n}$. It then follows ([11]), that $X$ is an $(n-d)$-dimensional PD-space and, replacing $X$ by a Euclidean regular neighborhood in $\mathbf{R}^{n+k-d}, k$ large, we see that $Y^{n+k}$ can be taken to be a manifold. Unfortunately, it is not then clear that $Y$ itself is triangulable. Moreover, even if $Y$ is triangulable there is no way, in general, of making the projection map strictly simplicial onto some triangulation of $X$.

These difficulties disappear if we assume that the structure group of the fibering

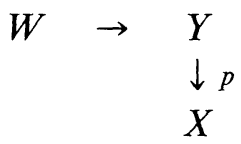


is the (finite) group of simplicial symmetries of $W$, (for some trangulation of $W$ ). In this case, we may assume that $Y$ is triangulated so that the projection map is simplicial, and the proof of 2.2.1 is immediately seen to entail the following result.

\subsubsection{Corollary. Let}

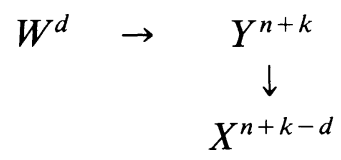

be as above, $(n-d \geq 5)$ and let $M^{n} \subset Y^{n+k}$ be embedded via a simple homotopy equivalence and topologically transverse to the triangulation. Let $M^{n}$ have a regular neighborhood $T$ so that

(1) $T \cap \sigma$ is a regular neighborhood of $M \cap \sigma$ for all simplices $\sigma$ of $Y$

(2) $T=p^{-1}(\mathrm{pT})$

Then there is a manifold $V^{n-d} \subset X$, the embedding a simple homotopy equivalence, so that $M^{n}$ is homeomorphic, consistent with the obvious homotopy equivalence, to the fiber space $p^{-1}\left(V^{n-d}\right)$.

Of course, the assumption that $p: Y \rightarrow X$ is simplicial is special. We may weaken this merely to the assumption that $Y$ is triangulated so that $p^{-1} \sigma$ is a subcomplex of $Y$ for each simplex $\sigma$ of $X$. A result is still obtainable under these weakened conditions. We carry over the hypotheses of 2.2.1 and 2.2.2, namely that the simple homotopy equivalence $M^{n} \subset Y^{n+k}$ is in topological general position with respect to the triangulation, that $M^{n}$ has a regular neighborhood $T$ in $Y$ with $T \cap \sigma$ a regular neighborhood of $M \cap \sigma$ for all simplices $\sigma$ of $Y$ and that $p^{-1}(\mathrm{pT})=T$. Following the proof of 2.2.1, we see that $U=p(T)$ is an $(n-d)$ dimensional PD space and that its embedding in $X$ is, in fact, pT. We conclude, therefore that there is a well-defined topological manifold structure $V^{n-d}$ on the homotopy type of $U$. However, we may no longer argue that $p^{-1}\left(V^{n-d}\right)$ is $M^{n}$, up to homeomorphism. For we have a $\nu(V)$ normal space $R^{n-d+1}$, with $\partial R=V \cup U$, rT embedded in $X \times I$ so as to connect the pT embedding $U \subset X$ and as embedding $V^{n-d} \subset X$ topologically transverse to the triangulation. However, we cannot conclude that $p^{-1} R$ is $\mathrm{rT}$ embedded in $Y \times I$ or even that $p^{-1} \cong \times W$ is $\mathrm{pT}$ embedded in $Y$. The difficulty is that $p$ is not simplicial. However, by the simplicial approximation theorem, we may assume (possibly having to subdivide the triangulation on $Y)$ that $p$ deforms to a simplicial map $q:(Y, \partial Y) \rightarrow$ $(X, \partial X)$ via a deformation $\Phi_{t}, \Phi_{0}=p, \Phi_{1}=q$. Moreover, by the covering homotopy property for locally-trivial fibrings, it follows that $\Phi_{t}$ may be covered by a deformation $\Psi_{t} ; Y \rightarrow Y$ with $\Psi_{0}=\mathrm{id}_{Y}$. 
2.2.3. Definition. Let $Z^{r}$ be a manifold and $g: Z^{r} \rightarrow Y$ a map. Say that $g$ is transverse to $M^{n}$ respecting $T$ if and only if it is topologically transverse to $M_{n}$ (in the sense of Scharlemann [17]) and, moreover, $g^{-1} T \rightarrow T$ is a topological bundle map covering $g^{-1} M \rightarrow M$.

We may then state our result

2.2.4. Corollary. Let $M^{n}, Y, X, V, p, T$ and the various triangulations be as above. Suppose $\Phi_{t}$ and its covering $\Psi_{t}$ can be chosen so that $\Psi_{t}$ is transverse to $M$ respecting $T$ on each simplex $\sigma$ of $Y$.

Then $M^{n}$ differs from $p^{-1}(V)$ at most by an action of $L_{n+1}\left(\pi_{1} M^{n}\right)$.

Proof. Recall that $U$ is in $X$ via a pT embedding. By the usual construction, one obtains a map $X \cup \partial X \rightarrow T(\nu(U))$ which is PT (and therefore RT) on each simplex; i.e. we obtain a transversality structure on $\nu(U)$, viz. a lifting $s: T(\nu(U)) \rightarrow \mathscr{Y}(\nu(U))$. Let $q=\Phi_{1} ; q$ is also PT on each simplex of $Y$, and thus we obtain a section

$$
\begin{array}{ccc} 
& & \mathscr{Y}(\nu(U)) \\
& s_{1} \nearrow & \downarrow \\
T\left(\nu_{Y}(M)\right) \stackrel{\rightarrow}{ } & T(\nu(U))
\end{array}
$$

which corresponds to a section

$$
T\left(\nu_{Y}(M)\right) \rightarrow \mathscr{Y}\left(\nu_{Y}(M)\right)
$$

However, by obvious geometric considerations, this is the same section induced by the fact that $\Psi_{1}:(Y, \partial Y) \rightarrow(Y, \partial Y)$ is transverse to $M$, respecting $T$, and thus, via $\Psi_{t}$ it is equivalent to the section determined by the original embedding of $M^{n}$ in $Y$. On the other hand, the section of $\mathscr{Y}(\nu(U)) \rightarrow T(\nu(U))$ determined by the pT embedding of $U$ is'equivalent, via $R$, to that determined by an embedding $V^{n-d} \subset X$ topologically transverse with respect to the triangulation; that is to say, we have two equivalent transversality structures on $\nu_{Y}(M)$; the first is determined by the given topological manifold structure on $M$, the second by taking the natural map $\nu_{Y}(M) \rightarrow \nu(U)$ and putting a transversality structure on $\nu(U)$ by putting the topological manifold structure $V$ on $U$. However, the latter structure on $\nu_{Y}(M)$ is easily seen to be equivalent to that obtained by identifying $M$, in the sense of simple homotopy type, with $p^{-1}(V)$. I.e. the corresponding TOP reductions of $\nu_{Y}(M)$ are identical. To summarize, then we may find a topological manifold $Q^{n+1} \subset Y \times I$ with $Q \cap$ $(Y \times\{1\})=Z, Q \cap(Y \times\{0\})=M, Z \cup M=\partial Q$, so that there is a topological normal map $\nu_{Y \times I}(Q) \rightarrow \nu_{Y}\left(M^{n}\right)$ covering the degree one map 
$(Q ; M, Z) \rightarrow(M \times I ; M \times\{0\}, M \times\{1\})$. This says, essentially, that the difference between $M$ and $Z$ in the structure set is at worst in the image of $L_{n+1}\left(\pi_{1} M\right)$.

2.2.5. Corollary. If $\pi_{1} M=0$, then under the hypothesis of 2.2.4, $M$ is the total space of a fibration over a manifold with fiber $W^{d}$.

2.2.6. Remark. A similar result to 2.2 .4 was proved in [5]. However, because of an incomplete understanding of the difference between intrinsic and extrinsic transversality structures, as well as inability to handle the difficulties of the non-simply connected case, inconvenient restrictions were made on $M^{n}$ and $W^{d}$, i.e. both were assumed to be 4-connected, and $(n-d)$ was assumed to be odd. Though the general idea of the present proof is the same, the present result is much stronger.

2.2.7. REMARK. The converse of 2.3 .1 and Corollary 2.2 .2 is obviously true: If $M^{n}$ is $V^{n-d} \times W^{d}$ (or a twisted product with a group of simplicial automorphisms as structure group) we may easily find $X, Y, T$ and the requisite triangulations with the stated properties. As for 2.2.4, the converse is at least true in essence; that is, if $M^{n}$ is strictly fibered by $W^{d}$, over $V^{n-d}$, and if the pullback $Y$ of the fibering to a Euclidean neighborhood $X$ of $V$ is triangulable as a manifold, then we may find $T$, and the deformations $\Phi_{t}, \Psi_{t}$ of the hypothesis of 2.2.4.

\section{The algebraic surgery space and the assembly map.}

3.1. The algebraic surgery space. In this chapter we study the algebraic surgery space with a view to understanding how a surgery obstruction may be seen to arise from a section $\mathscr{Y}(\nu) \stackrel{s}{\rightarrow} T(\nu)$ where $\nu$ is the Spivak normal fibration of a Poincaré duality space $X^{n}$, and $s$ is envisioned more geometrically as a simplicial map from a triangulated $W / \partial W$ to $\mathscr{Y}(\nu), W$ being the regular neighborhood of $X^{n}$ in $\mathbf{R}^{n+k}$. It is seen, since such a section specifies a topological reduction of $\nu$, that there is a well-defined surgery obstruction in $L_{n}\left(\pi_{1}(X)\right)$ defined via the classical procedure of constructing a degree-one normal map $M^{n} \rightarrow X^{n}, M^{n}$ a topological manifold. But we wish to establish that this obstruction arises naturally on the "chain level", i.e. that the specific map $W / \partial W \rightarrow \mathscr{Y}(\nu)$ defines a specific Q.A.P.C. over $\mathbf{Z}\left[\pi_{1}(X)\right]$ whose bordism class, in the Q.A.P.C. category, is an element of $L_{n}\left(\pi_{1}(X)\right.$ ) (and, in particular, the surgery obstruction obtained classically via the corresponding topological reduction of $\nu$ ). This is, in essence, the content of Lemma 1.2.1 whose proof will be given in 3.2 below. 
The most important ingredient in our understanding of surgery theory from an algebraic and combinatorial point of view is the surgery space $\mathbf{L}_{k}(A)$ where $A$ is an associative ring with unit and involution ${ }^{-}: A \rightarrow A$; $a \mapsto \bar{a}$ with $\overline{\bar{a}}=a,(\overline{a+b})=\bar{a}+\bar{b}, \overline{a b}=\bar{b} \cdot \bar{a}$. Typically, $A$ will be either $\mathbf{Z}=\mathbf{Z}[\{1\}]$, with trivial involution, or the group $\operatorname{ring} \mathbf{Z}\left[\pi_{1}(X)\right], X$ a fixed P.D. space, and - arising from the group inverse on $\pi_{1}(X)$.

We consider left $A$-modules $M$ over $A$. The dual $M^{*}$ of $M$ is $\operatorname{Hom}_{A}(M, A)$ where the $A$-module structure is specified by

$$
A \times M^{*} \rightarrow M^{*} ; \quad(a, f) \mapsto(x \mapsto f(x) \bar{a}) .
$$

If $N$ is another $A$-module we have dual $A$-module morphisms defined by

$$
\text { *: } \operatorname{Hom}_{A}(M, N) \rightarrow \operatorname{Hom}_{A}\left(N^{*}, M^{*}\right) ; \quad f \mapsto\left(f^{*}: g \mapsto g(f(x))\right) .
$$

If we have a chain complex of $A$-modules

$$
C: \cdots \stackrel{d}{\rightarrow} C_{r+1} \stackrel{d}{\rightarrow} C_{r} \stackrel{d}{\rightarrow} C_{r-1} \stackrel{d}{\rightarrow} \cdots \quad\left(r \in \mathbf{Z}, d^{2}=0\right)
$$

we have the usual homology and cohomology $A$-modules

$$
\begin{aligned}
& H_{r}(C)=\operatorname{ker}\left(d: C_{r} \rightarrow C_{r-1}\right) / \operatorname{im}\left(d: C_{r+1} \rightarrow C_{r}\right) \text { and } \\
& H^{r}(C)=\operatorname{ker}\left(d^{*}: C_{r}^{*} \rightarrow C_{r+1}^{*}\right) / \operatorname{im}\left(d^{*}: C_{r-1}^{*} \rightarrow C_{r}^{*}\right) .
\end{aligned}
$$

It will be convenient to have a dimension shifting operator on chain complexes, i.e. given a chain complex $C$ and $k \in \mathbf{Z}$ we obtain a formally distinct chain complex $S^{k} C$ with $\left(S^{k} C\right)_{r}=C_{r-k}$ and $d:\left(S^{k} C\right)_{r} \rightarrow$ $\left(S^{k} C\right)_{r-1}$ identified with $d: \quad C_{r-k} \rightarrow C_{r-k-1}$. Trivially $H_{*}\left(S^{k} C\right)=$ $H_{*-k}(C), H^{*}\left(S^{k} C\right)=H^{*-k}(C)$. An $A$-module chain complex $C$ is said to be $m$-dimensional, $m \geq 0$, iff $C_{r}$ is a free f.g. $A$-module for each $r$, with $C_{r}=0$ for $r>m$ and $r<0$.

Given $A$-modules $M, N$ let $M \otimes_{A} N$ denote, for our purposes, the Z-module $M \otimes_{\mathbf{Z}} N /\{x \otimes a y-\bar{a} x \otimes y\}$. Extending to $A$-module chain complexes we obtain from two such, $C$ and $D$, the $\mathbf{Z}$-module chain complex $C \otimes_{A} D$ where $\left(C \otimes_{A} D\right)_{r}=\sum_{p+q=r} C_{p} \otimes_{A} D_{q}$ and $d:\left(C \otimes_{A} D\right)_{r}$ $\rightarrow\left(C \otimes_{A} D\right)_{r-1} ; x \otimes y \mapsto x \otimes d y+(-)^{q} d x \otimes y\left(x \in C_{p}, y \in D_{q}, p+q\right.$ $=r)$.

Given $C$, an $A$-module chain complex, define $Q_{m}(C)$ as the homology group

$$
H_{m}\left(W \otimes_{\mathbf{z}\left[\mathbf{z}_{2}\right]}\left(C \otimes_{A} C\right)\right) .
$$

Here, $C \otimes_{A} C$ is a $\mathbf{Z}\left[\mathbf{Z}_{2}\right]$-module chain complex by letting the generator $T \in \mathbf{Z}_{2}$ act on $C \otimes_{A} C$ by transposition, i.e. $T(x \otimes y)=(-)^{p q}(y \otimes x) \in$ $C_{q} \otimes_{A} C_{p}$ when $x \in C_{p}, y \in C_{q} . W$ is the $\mathbf{Z}\left[\mathbf{Z}_{2}\right]$-module chain complex

$$
W: \cdots \rightarrow \mathbf{Z}\left[\mathbf{Z}_{2}\right]^{1+T} \rightarrow \mathbf{Z}\left[\mathbf{Z}_{2}\right] \stackrel{1-T}{\rightarrow} \mathbf{Z}\left[\mathbf{Z}_{2}\right] \rightarrow 0
$$

(i.e. all terms in non-negative dimensions are $\mathbf{Z}\left[\mathbf{Z}_{2}\right]$, all negative dimensional terms vanish, the differential operator is alternately $1+T$ and 
$1-T)$. A given element $\psi \in Q_{m}(C)$ may be represented by a collection of chains $\left\{\psi_{s} \in\left(C \otimes_{A} C\right)_{m-s} \mid s \geq 0\right\}$ satisfying the relations

$$
d\left(\psi_{s}\right)=(-)^{m-s}\left(\psi_{s+1}+(-)^{s+1} T \psi_{s+1}\right) \in\left(C \otimes_{A} C\right)_{m-s-1} \quad(s \geq 0) .
$$

Thus, in particular, $(1+T) \psi_{0} \in\left(C \otimes_{A} C\right)_{m}$ is a cycle representing a homology class in $H_{m}\left(C \otimes_{A} C\right)$.

An $m$-dimensional quadratic complex over $A$ is a pair $(C, \psi)$, where $C$ is an $m$-dimensional $A$-module chain complex and $\psi \in Q_{m}(C)$. The fundamental maps of $(C, \psi)$ are the $A$-module morphisms $(1+T) \psi_{0}$ : $H^{r}(C) \rightarrow H_{m-r}(C)$. On the level of cocycles and cycles, this map is defined by $[f] \mapsto \Sigma[\overline{f(x)} \cdot y]$ where $f$ is a cocycle of $C_{r}^{*}$ and $x \in C_{r}$, $y \in C_{m-r}$ such that $(1+T) \psi_{0}=\sum x \otimes y$.

We call the quadratic complex $(C, \psi)$ Poincare iff the fundamental maps are isomorphisms.

There are two fundamental ways in which quadratic Poincaré complexes arise in the context of this paper.

3.1.1. EXAMPLE. Let $\xi^{k}$ be an oriented spherical fibration over a space $B$ and let $A=\mathbf{Z}\left[\pi_{1}(B)\right]$. Let $X$ be a $\xi$-normal space. Then we obtain a quadratic Poincaré complex $\Phi=(C, \psi)$ which measures the failure of $X$ to be a Poincaré complex over $A$, with $C=S^{-1} C([X] \cap-)$ the algebraic mapping cone with a dimension shift of the chain map $[X] \cap-: C(\tilde{X})^{n-*}$ $\rightarrow C(\tilde{X})$ with $\tilde{X}$ the pullback to $X$ of the universal cover $\tilde{B}$ of $B$. See [16, $\S 7.3]$ for further details.

3.1.2. ExAmple. Let $f: X \rightarrow Y$ be a degree-one normal map between oriented Poincaré spaces. Then the classical "kernel complex" arises, in fact, from an $n$-dimensional quadratic Poincaré complex $(C, \psi)$ over $\mathbf{Z}\left[\pi_{1}(Y)\right]=A$, with $C$ the algebraic mapping cone of the Umkehr $A$-module chain map

$$
f^{!}: C(\tilde{Y}) \stackrel{([Y] \cap-)^{-1}}{\stackrel{\rightarrow}{\leftrightarrows}} C(\tilde{Y})^{n-*} \stackrel{\tilde{f}^{*}}{\rightarrow} C(\tilde{X})^{n-*} \stackrel{[X] \cap-}{\rightarrow} C(\tilde{X})
$$

where $\tilde{Y}$ is the universal cover of $Y$ and $\tilde{X}$ is the pullback cover of $X$. See $[15, \S 4]$ for further details.

Let us now consider pairs $(C, D)$ of $A$-module chain complexes (i.e. $D$ a subcomplex of $C$ ) such that each $D_{r}$ is a direct summand of $C_{r}$. We obtain a pair of $\mathbf{Z}$-module chain complexes

$$
\left(W \otimes_{\mathbf{z}\left[\mathbf{z}_{2}\right]}\left(C \otimes_{A} C\right), W \otimes_{\mathbf{z}\left[\mathbf{z}_{2}\right]}\left(D \otimes_{A} D\right)\right)=\left(W_{C}, W_{D}\right) .
$$


Define the relative $Q$-groups of $(C, D)$ to be the relative homology groups

$$
Q_{m}(C, D)=H_{m}\left(W_{C}, W_{D}\right)
$$

which fit into an exact sequence

$$
\cdots \rightarrow Q_{m}(D) \rightarrow Q_{m}(C) \rightarrow Q_{m}(C, D) \rightarrow Q_{m-1}(D) \rightarrow \cdots
$$

in the obvious way.

An element $\psi^{C, D}$ of $Q_{m}(C, D)$ is represented by a collection

$$
\left\{\left(\psi_{s}^{C}, \psi_{s}^{D}\right) \in\left(C \otimes_{A} C\right)_{m-s} \oplus\left(D \otimes_{A} D\right)_{m-s-1} \mid s \geq 0\right\}
$$

satisfying the compatibility conditions

$$
\begin{aligned}
d\left(\psi_{s}^{C}\right) & =(-)^{m-s}\left(\psi_{s+1}^{C}+(-)^{s} T \psi_{s+1}^{C}\right)+\psi_{s}^{D} \\
& \in\left(D \otimes_{A} D\right)_{m-s-1} \subseteq\left(C \otimes_{A} C\right)_{m-s-1} .
\end{aligned}
$$

Thus the connecting homomorphism $Q_{m}(C, D) \rightarrow Q_{m-1}(D)$ in the long exact sequence for $Q$-groups above is given simply by $\psi^{C, D}=\left[\left\{\psi_{s}^{C}, \psi_{s}^{D}\right\}\right]$ $\mapsto\left[\left\{\psi_{s}^{D}\right\}\right]$. Also, $(1+T) \psi_{0}^{C, D} \in H_{m}\left(C \otimes_{A} C, D \otimes_{A} D\right)$ is a homology class representing a relative cycle of $H_{m}\left(C \otimes_{A} C, D \otimes_{A} D\right)$.

An m-dimensional quadratic pair $\left(C, D, \psi^{C, D}\right)$ consists of a pair of $A$-module chain complexes $(C, D), C m$-dimensional, $D(m-1)$ dimensional, and $\psi^{C, D} \in Q_{m}(C, D)$. The fundamental maps of $\left(C, D, \psi^{C, D}\right)$ are the $A$-module morphisms $(1+T) \psi_{0}^{C, D}: H^{r}(C) \rightarrow$ $H_{m-r}(C, D)$.

We shall say that the quadratic pair $(C, \partial C, \psi)$ is Poincare if the fundamental maps are isomorphisms, in which case the boundary $(\partial C, \partial \psi)$ is an $(m-1)$-dimensional quadratic Poincaré complex.

If $\Phi=(C, \psi)$ is a quadratic Poincaré complex then, by definition, $-\Phi=(C,-\psi)$. We say that $(C, \psi),\left(C^{\prime}, \psi^{\prime}\right)$ are bordant as $m$-dimensional quadratic Poincaré complexes over $A$ if there is an $(m+1)$-dimensional quadratic Poincaré pair over $A,(B, \partial B, \phi)$ so that $(\partial B, \partial \phi)=$ $\left(C \oplus C^{\prime}, \psi \oplus-\psi^{\prime}\right)$.

3.1.3. Proposition. Bordism is an equivalence relation among $m$-dimensional quadratic Poincaré complexes. The set of bordism classes forms an abelian group $L_{m}^{h}(A)$ with addition by

$$
[(C, \psi)]+\left[\left(C^{\prime}, \psi^{\prime}\right)\right]=\left[\left(C \oplus C^{\prime}, \psi \oplus \psi^{\prime}\right)\right]
$$

Proof. See $[14, \S 3]$.

We shall now expand the notion of quadratic Poincare pair to encompass objects with a more elaborate combinatorial decomposition. As a heuristic aid, imagine a manifold $M^{m}$ embedded in the standard $n$-simplex $\Delta^{n}(n>m)$ with $M^{m} \cap \dot{\Delta}^{n}=\partial M^{m}$, and $\partial M^{m}$ intersecting all 
faces $\alpha$ of $\Delta^{n}$ transversally, so that if $n-m=j$ and $\alpha$ is an $r$-dimensional face, then $M_{\alpha}=M^{m} \cap \alpha$ is an $(r-j)$-manifold (possibly void) with $\partial M_{\alpha}=\bigcup_{\beta<\alpha} M_{\beta}$. The idea is to replace "manifold" with "quadratic Poincaré complex" while keeping intact the combinatorial pattern of the decomposition.

Accordingly, we say, formally that an $n$-ad of $A$-module chain complexes is an $A$-module chain complex $X=C\left(\Delta^{n}\right)$ together with a collection of subcomplexes $C(\alpha)\left(\alpha \leq \Delta^{n}\right)$ such that $C(\alpha \cap \beta)=C(\alpha) \cap$ $C(\beta)$. We say that the $n$-ad $C=\left\{C(\alpha) \mid \alpha \leq \Delta^{n}\right\}$ is $m$-dimensional iff each $C(\alpha)$ is a $(\operatorname{dim} \alpha-n+m)$-dimensional f.g. free $A$-module chain complex, with $C(\beta)$ a direct summand of $C(\alpha)$ whenever $\beta<\alpha$.

We adopt the notation $C(\dot{\alpha})=\oplus_{\beta<\alpha} C(\beta) / \sim$ where the equivalence relation identifies $x \in C(\gamma)$ with its image in $C(\beta)$ whenever $\gamma<\beta$. Thus $C(\alpha)$ is a $(\operatorname{dim} \alpha-m+n-1)$-dimensional f.g. free $A$-module chain complex.

Thus, a quadratic $m$-dimensional $n$-ad is a pair $\{C, \bar{\psi}\}$, where $\bar{\psi}$ is a collection of the form $\left\{\psi^{\alpha}\right\}, \alpha \leq \Delta^{n}$ with $\psi^{\alpha} \in Q_{\operatorname{dim} \alpha-n+m}(C(\alpha), C(\dot{\alpha}))$, with each $\psi^{\alpha}$ being represented by a pair of collection of cycles

$$
\left\{\left(\psi_{s}^{\alpha}, \psi_{s}^{\dot{\alpha}}\right) \in\left(C(\alpha) \otimes_{A} C(\alpha)\right)_{m-s} \oplus\left(C(\dot{\alpha}) \otimes_{A} C(\dot{\alpha})\right)_{m-s-1} \mid s \geq 0\right\}
$$

such that $\psi_{s}^{\dot{\alpha}}$ is the image of

$$
\bigoplus_{\substack{\beta<\alpha \\ \beta=\operatorname{dim} \alpha-1}}^{\beta} \psi_{s}^{\beta} \in \bigoplus_{\substack{\beta<\alpha \\ \operatorname{dim} \beta-\operatorname{dim} \alpha-1}} C(\beta) \text { in } C(\dot{\alpha}),
$$

with each $\left(C(\alpha), C(\dot{\alpha}), \psi^{\alpha}\right)$ a quadratic pair.

We say that $(C, \bar{\psi})$ is Poincaré iff each $\left(C(\alpha), C(\dot{\alpha}), \psi^{\alpha}\right)$ is a Poincaré pair.

Note that if $\alpha$ is an $(n-r)$-dimensional face of $\Delta^{n}$, then, given an $m$-dimensional Poincaré $n$-ad over $A(C, \bar{\psi})$ we obtain a derived $(m-r)$ dimensional $(n-r)$-ad $d_{\alpha}(C, \bar{\psi})$, by identifying $\alpha$ with the standard simplex $\Delta^{n-r}$ in the canonical way, and letting $C\left(\beta^{\prime}\right)=C(\beta)$, whenever $\beta^{\prime}$ is a face of $\Delta^{n-r}$ corresponding to the face $\beta<\alpha$ under this identification. This leads naturally to a semi-simplicial construction, viz. an s.s. complex $\mathbf{L}_{k}^{h}(A)(k \in \mathbf{Z})$ with $m$-simplexes the $(m+k)$-dimensional Poincare $m$-ads over $A(C, \bar{\psi})$. From this point of view 3.1.3 translates to:

\subsubsection{Proposition. Up to natural isomorphism}

$$
\pi_{m}\left(\mathbf{L}_{k}^{h}(A)\right)=\left\{\begin{array}{ll}
L_{m+k}^{h}(A) & \text { if } m+k \geq 0 \\
0 & \text { if } m+k<0
\end{array} \quad(m \geq 0, k \in \mathbf{Z}) .\right.
$$

Given 3.1.3, the proof of 3.1.4 is a completely routine s.s. argument provided one knows the following fact: 
3.1.5. Proposition. The s.s. complex $\mathbf{L}_{k}^{h}(A)$ satisfies the Kan extension condition.

(The original geometric L-spaces of Quinn [9] satisfied the Kan extension condition on account of a formal procedure which adjoins enough simplices to a s.s. complex to make it Kan. The algebraic $\mathbf{L}$-spaces were first defined in [12], using forms and formations, and the Kan extension condition was verified using an algebraic glueing operation (cf. $[16, \S 1.8])$. These $\mathbf{L}$-spaces are deformation retracts of $\mathbf{L}_{k}^{h}(A)$, since highly-connected quadratic Poincaré complexes are the same as forms and formations, and it is always possible to perform quadratic Poincaré surgery below the middle dimension.)

The essential meaning of 3.1.5 is as follows: suppose we have an open box in $\mathbf{L}_{k}$, i.e. a subcomplex $K$ of $\mathbf{L}_{k}(A)$ consisting of $m+1 m$-simplices of $\mathbf{L}_{k}(A)$ which is abstractly isomorphic to $\dot{\Delta}^{m+1}$ with the leading face deleted. Then the Kan condition specifies that there is an $(m+1)$-simplex of $\mathbf{L}_{k}(A) m+1$ of whose faces constitute $K$. This, in turn, means essentially that we may form an $(m+k)$-dimensional $m$-ad $(C(K), \bar{\psi}(K))$ where $C(K)$ is a quotient of the direct sum $\oplus_{\tau \subseteq K} C_{\tau}$, modulo the obvious identifications. This "assembly" construction generalizes a bit further, and this is the heart of the matter in proving 3.1.4. Given a map $f: S^{m} \rightarrow \mathbf{L}_{k}(A)$ we may assume it is simplicial with respect to some triangulation of $S^{m}$. Thus for each $n$-simplex $\tau$ of $S^{m}$ we obtain an $(n+k)$-dimensional quadratic Poincaré $n$-ad over $A f(\tau)=(C(\tau, \bar{\psi}(\tau))$. The quotient of the direct sum $\bigoplus_{\tau, \operatorname{dim} \tau=m} C_{\tau}$ modulo the obvious identifications is an $(m+k)$-dimensional $A$-module chain complex $C(f)$ with an $(m+k)$ dimensional quadratic Poincaré duality structure $\psi(f)$. The relative version of this assembly argument shows that the bordism class of $(C(f), \psi(f))$ depends only on the homotopy class of $f$. It is in fact easily seen that the bordism class of $(C(f), \psi(f))$ completely determines the homotopy class of $f$. This, together with the additional fact that every bordism class is realized by such a map, gives the isomorphism of Theorem 3.1.4.

It should be noted that the gist of the argument works as well if $S^{m}$ is replaced by any oriented PL manifold $M^{m}$ i.e. given a simplicial map $f$ : $M^{m} \rightarrow \mathbf{L}_{k}^{h}(A)$ the images of the $m$-simplices of $M$ may be assembled together to define a closed $(m+k)$-dimensional quadratic Poincaré complex over $A$. Thus, in particular, we have a splitting $\Omega_{m}^{P L}\left(\mathbf{L}_{k}^{h}(A)\right) \rightarrow$ $\pi_{m}\left(\mathbf{L}_{k}^{h}(A)\right)$ of the forgetful map, which for $A=\mathbf{Z}$ is essentially the well-known splitting $\Omega_{m}^{P L}(G / \mathrm{TOP}) \rightarrow \pi_{m}(G / \mathrm{TOP})=L_{m}(\mathbf{Z})$ (since $\mathbf{L}_{0}(\mathbf{Z})$ $=L_{0}(\mathbf{Z}) \times G /$ TOP, cf. [8]). 
The above discussion would suffice had we merely been interested (in $\S 1$ ) in recognizing when a PD-space $X$ is a manifold up to homotopy type rather than simple homotopy type, and in classifying such structures up to $h$-cobordism rather than $s$-cobordism. To deal with such finer questions we must use the simple quadratic $L$-groups $L_{*}^{s}(A)$ instead of $L_{*}^{h}(A)$.

Accordingly, we make the following modifications. Rather than dealing with finite dimensional $A$-module chain complexes we consider such complexes together with a preferred class of $A$-bases in each dimension, and in dealing with quadratic Poincaré complexes $(C, \psi)$ we require the Poincaré duality chain equivalence $(1+T) \psi_{0}: C^{n-*} \rightarrow C$ to be simple, i.e. to have zero torsion in the Whitehead group $\mathrm{Wh}(\pi)$ if $A=\mathbf{Z}[\pi]$. N.B. We reserve the terminology Q.A.P.C. for such objects.

3.1.6. Remark. We shall elaborate Examples 3.1 .1 and 3.1 .2 to the based case. In 3.1.1 we pointed out that given a $\xi$-normal space $X^{m}$ there is a well-defined (unbased) $(m-1)$-dimensional quadratic Poincaré complex $\Phi$ over $Z\left[\pi_{1}(B)\right]$ ( $B=$ base of $\xi$ ). We claim that in fact $\Phi$ is naturally endowed with the structure of a (based) QAPC, since the underlying chain complex $C=S^{-1} C([X] \cap-)$ is the algebraic mapping cone with a dimension shift of the chain map $[X] \cap-: C(\tilde{X})^{m-*} \rightarrow C(\tilde{X})$ of based $\mathbf{Z}\left[\pi_{1}(B)\right]$-module chain complexes with respect to which $(1+T) \psi_{0}$ has the simple $\left(\begin{array}{rr}0 & 1 \\ \pm & 0\end{array}\right)$. If $f: X \rightarrow Y$ is a normal map between oriented Poincaré duality spaces such that the Poincaré duality chain equivalences $[X] \cap-: C(\tilde{X})^{m-*} \rightarrow C(\tilde{X}),[Y] \cap-: C(\tilde{Y})^{m-*} \rightarrow$ $C(\tilde{Y})$ are simple then the kernel complex is a (based) QAPC.

We may carry over the other elaborations of the idea of unbased quadratic complex to the world of (based) QAPC's. For example, a QAPC pair may be defined as a quadratic pair $(C, \partial C, \psi)$ where there are chosen bases for $C$ and $\partial C$, with the base of $C$ extending that of $\partial C$, with the Poincaré duality chain equivalence $(1+T) \psi_{0}:(C / \partial C)^{m-*} \rightarrow C$ simple. It follows that we may speak of the bordism group of QAPC's. By analogy with the $L^{h}$-case (Proposition 3.1.3) we have:

3.1.7. Proposition. The group of bordism classes of m-dimensional QAPC's over $A$ is isomorphic to the Wall group $L_{m}^{s}(A)$.

Proof. See [14].

By the same token, we may speak of $m$-dimensional $n$-ads of QAPC's over $A$, i.e. $n$-ads $(C, \psi)$ so that the chosen basis for $C(\alpha)$ is a subset of that for $C(\beta)$ whenever $\alpha<\beta \leq \Delta^{n}$, and such that the Poincare duality 
chain equivalences are simple. We may therefore construct s.s. complexes $\mathbf{L}_{k}^{s}(A)(k \in \mathbf{Z})$ (satisfying the Kan condition) such that:

\subsubsection{Proposition. Up to natural isomorphism}

$$
\pi_{m}\left(\mathbf{L}_{k}^{s}(A)\right)=\left\{\begin{array}{ll}
L_{m+k}^{s}(A) & \text { if } m+k \geq 0 \\
0 & \text { if } m+k \leq 0
\end{array} \quad(m \geq 0, k \in \mathbf{Z}) .\right.
$$

The outline of the proof of 3.1.4 will serve as well for 3.1.8 above. The problem of keeping track of a preferred class of $A$-bases adds only slight technical complications. Note that, as in the case of unbased quadratic complexes, we may, given a simplicial map $f: M^{m} \rightarrow \mathbf{L}_{k}^{s}(A), M^{m}$ a $\mathrm{PL}$ manifold, assemble an $(m+k)$-dimensional QAPC $(C(f), \psi(f))$ over $A$.

This assembly procedure may be elaborated still further and it is this elaboration which is the core of the following $\$ 3.2$.

3.2. The assembly map. Recall the situation posited in 1.21: we have an orientable PD-space $X$, a regular neighbourhood $W$ of $X$ in $\mathbf{R}^{n+k}$ (so that $\partial W \subset W$ represents the Spivak normal fibration $\xi^{k}$ of $X$ ) and a triangulation of $W$. We also have a map $g: W \cup c \partial W \rightarrow T\left(\xi^{k}\right)$, homotopically the natural homotopy equivalence of these spaces, which is in fact RT on each simplex of $W \cup c \partial W$. We wish to obtain from these data an explicit $n$-dimensinoal QAPC over $\mathbf{Z}\left[\pi_{1}(X)\right] \tilde{\Phi}(g)$. This procedure is the assembly map. (The motivation for this nomenclature will shortly become evident.)

To construct $\tilde{\Phi}(g)$ we begin by recalling some facts about normal spaces, their associated chain complexes, and the (simple) Poincaré duality obstruction of such spaces. Let $f: M^{n+k} \rightarrow T\left(\xi^{k}\right)$ be an NT map where $M^{n+k}$ is an orientable manifold and $\xi^{k}$ is an orientable $S^{k-1}$-fibration over a space $B$. In general $f^{-1} \mathscr{M}\left(\xi^{k}\right)=X$ is not even a simple (= integral) coefficient Poincaré duality space. But this deficiency may be precisely measured.

Let $[X] \in H_{n}(X ; \mathbf{Z})$ be the image of $[M] \in H_{n+k}(M ; \mathbf{Z})$ under the composite $H_{n+k}(M ; \mathbf{Z}) \rightarrow \tilde{H}_{n+k}\left(T\left(g^{*} \xi\right) ; \mathbf{Z}\right) \stackrel{\sim}{\rightarrow} H_{n}(X ; \mathbf{Z})(g=f \mid: X \rightarrow$ $\left.\eta\left(\xi^{k}\right)\right)$. The algebraic mapping cone with a dimension shift $S^{-1} C([X] \cap-$ : $\left.C(X)^{n-*} \rightarrow C(X)\right)$ supports an $(n-1)$-dimensional quadratic structure $\psi \in Q_{n-1}\left(S^{-1} C([X] \cap-)\right)([16$, Prop. 7.4.1]), such that

$$
R_{f}=\left(S^{-1} C([X] \cap-), \psi\right)
$$

is an $(n-1)$-dimensional quadratic Poincaré complex over $\mathbf{Z}$. (Strictly speaking, $S^{-1} C([X] \cap-)$ may fail to be $(n-1)$-dimensional, since $[X] \cap$ -: $H^{n}(X ; \mathbf{Z}) \rightarrow H_{0}(X ; \mathbf{Z})$ may fail to be onto. However, this can be achieved either by normal space surgery on the 0 -cells, or else by quadratic 
Poincaré surgery.) We remark that $R_{f}$ is well-defined up to equivalence (i.e. chain homotopy equivalence preserving the quadratic structure) in the world of quadratic Poincaré complexes over $\mathbf{Z}$.

Suppose, now, we are given a particular quadratic Poincaré pair $J$ such that $\partial J=R_{f}$. Then $J$ determines a sequence of quadratic Poincaré surgeries from $R_{f}$ to 0 ([14, Prop. 4.1]), which in turn determine an algebraic normal bordism from $C(X)$ with its symmetric structure to an $n$-dimensional symmetric Poincaré complex $\Gamma_{*}$ over $\mathbf{Z}$, together with a chain map $\Gamma_{*} \rightarrow C_{*}(B)$. We leave aside at this point the interesting (but not directly relevant) question of whether $J$ and this algebraic normal bordism may be realized geometrically, i.e. whether there is a homotopy $F$ of $f$ to $f_{0}$, which is NT, such that $R_{f_{0}}=0, R_{F}=J$ and $X_{0}=f_{0}^{-1}\left(\eta\left(\xi^{k}\right)\right)$ is a simple coefficient Poincaré duality space such that $\Gamma_{*}=C_{*}\left(X_{0}\right)$ up to chain homotopy, although the theory of surgery on normal and Poincaré spaces announced by Quinn [11] implies that such is the case.

Now let us generalize this situation to poly-ads. Suppose $f$ is now a map $\Delta^{n+k} \rightarrow T\left(\xi^{k}\right)$ which is RT, as is the restriction of $f$ to any face of $\Delta^{n+k}$. For each face $\alpha \leq \Delta^{n+k}$ we have the normal space $X$ of dimension $\operatorname{dim} \alpha-k$, and we thus obtain an $(n+k)$-ad $R_{f}=\left\{R_{\sigma}\right\}_{\sigma \leq \Delta^{n+k}}$ of quadratic Poincaré complexes over $\mathbf{Z}$, where $\operatorname{dim} R_{\sigma}=\operatorname{dim} X_{\sigma}-1$. Suppose that $R_{f}$ is the boundary of an $(n+k)$-ad $J=\left\{J_{\sigma}\right\}$ of quadratic Poincaré pairs over $\mathbf{Z}$ with $\partial J_{\sigma}=R_{\sigma} \cup \cup_{\tau \leq \dot{\sigma}} J_{\tau}$. Then $J$ determines a sequence of $(n+k)$-ad quadratic Poincaré surgeries from $R_{f}$ to 0 , which in turn determine an $(n+k)$-ad algebraic normal bordism from $\left\{C_{*}\left(X_{\sigma}\right)\right\}$ to an $(n+k)$-ad of symmetric Poincaré complexes over $\mathbf{Z},\left\{\Gamma_{*}\left(X_{\sigma}\right)\right\}$, together with compatible chain maps $\Gamma_{*}\left(X_{\sigma}\right) \rightarrow C_{*}(B)$.

Returning to the case where $f: M^{n+k} \rightarrow T(\xi)$, assume also that $M^{n+k}$ is a combinatorially triangulated manifold and that $f$ is simplex-wise RT. We now have, for each $j$-simplex $\sigma$ of $M^{n+k}$ the $j$-ad of normal spaces $\left\{f^{-1}\left(\mathscr{M}\left(\xi^{k}\right)\right) \cap \tau\right\}_{\tau \leq \sigma}$ and $j$-ad of quadratic Poincaré complexes over $\mathbf{Z}\left\{R_{\tau}\right\}_{\tau \leq \sigma}$ where $R_{\tau}=R_{f \mid \tau}$. Additionally, we have the $j$-ad of quadratic Poincaré pairs over $\mathbf{Z}\left\{J_{\tau}\right\}_{\tau \leq \sigma}$ with boundary $\left\{R_{\tau}\right\}_{\tau \leq \sigma}$. Finally, we obtain the $j$-ad of symmetric Poincaré complexes over $\mathbf{Z},\left\{\Gamma_{\tau}\right\}_{\tau \leq \sigma}$.

3.2.0. Assembly Lemma.

(i) $\cup_{\sigma} R_{\sigma}$ is an $(n-1)$-dimensional quadratic Poincaré complex over $\mathbf{Z}$ which is canonically chain homotopy equivalent to $R_{f}$.

(ii) $\cup_{\sigma} J_{\sigma}=J$ is an n-dimensional quadratic Poincaré pair over $\mathbf{Z}$ with boundary $\partial J=R_{f}$

(iii) $\cup_{\sigma} \Gamma_{\sigma}$ is an $n$-dimensional symmetric Poincaré complex over $\mathbf{Z}$ canonically homotopy equivalent to that produced from $R_{f}$ by the algebraic 
normal surgery with trace $J$, and there is a chain map $\cup_{\sigma} \Gamma_{\sigma} \rightarrow$ $C(B)$.

We indicate the main conceptual ideas of the proof, concentrating on part (i), but with equal applicability to (ii) and (iii) with some addenda. (The assembly of symmetric Poincaré $n$-ads over PL manifolds was previously described by Mishchenko and Solov'ev [8].) The key point may be rephrased thus:

Suppose $V^{r}$ is a compact, orientable triangulated PL manifold (without boundary), and that there is an assignment $\alpha: \sigma \mapsto \alpha(\sigma)$ which to any $j$-simplex $\sigma$ of $V$ associates a $(j+q)$-dimensional $j$-ad $\alpha(\sigma)$ of quadratic Poincaré complexes over $\mathbf{Z}$, the assignment being consistent with the face relations. This might also be thought of as a simplicial map $V \rightarrow \mathbf{L}_{q}(\mathbf{Z})$, or else as a $q$-dimensional algebraic mock bundle over $V$ of quadratic Poincaré $n$-ads over $\mathbf{Z}$. The assembly Lemma 3.2 .0 is the algebraic analogue of the result of Buoncristiano, Rourke and Sanderson [2, Lemma II.1.2] that the total space of a $q$-dimensional geometric mock bundle over an $r$-dimensional PL manifold is a $(q+r)$-dimensional PL manifold. The heart of our assembly lemma is that to each $(V, \alpha)$ we can glue all the quadratic Poincaré $n$-ads $\alpha(\sigma)$ together to define a closed $(q+r)$ dimensional Q.A.P.C. $T_{\alpha}$, which is uniquely determined by to chain homotopy equivalence.

(If we wish to consider manifolds $V$ with boundary, the basic idea is easily modified to produce a Q.A.P.C. $T_{\alpha}$ with boundary $T_{\alpha \mid \partial V}$.)

Our method of approach to the construction of $T_{\alpha}$ is this: we first need:

3.2.1. LeMMA. Let $A^{i}$ be a closed $i$-dimensional Q.A.P.C. over $\mathbf{Z}$ and let $K^{j}$ be a regular cell decomposition of $a$ (closed) oriented j-dimensional PL manifold. Then there is a well-defined $(i+j)$-dimensional Q.A.P.C. $A \times K$ over $\mathbf{Z}$ whose underlying chain complex is $A \otimes C(K)$, where $C(K)$ denotes the integral cellular chains on $K$.

Proof. This is a particular case of the products of $[14, \S 8]$.

By a slight extension we can obtain:

3.2.2. Lemma. Let $A$ now denote a p-ad of Q.A.P.C.'s of dimension $i$, and let $K^{j}$ be as above. Then there is a well-defined p-ad $A \times K$ of dimension $(i+j)$. 
Next, for conceptual reasons which are not, strictly speaking, formally necessary, we view the manifold $V$ as having associated to its triangulation an additional decomposition, viz., a handlebody structure with one $j$-handle $H\left(\sigma^{j}\right)$ for each $j$-simplex $\sigma^{j}$ of $V$. We visualize $H\left(\sigma^{j}\right)$ as having for core disc a shrunken copy $\bar{\sigma}^{j}$ of $\sigma^{j}$, with $\bar{\sigma}^{j} \subset$ int $\sigma^{j}$. Moreover, it is convenient to view the collection of handles $\left\{H\left(\sigma^{j}\right)\right\}_{j \leq r, \sigma^{j} \in V}$ as a collection of subcomplexes of an even finer cell decomposition of $V$. In particular, consider for each $\sigma^{j}$ the abstract simplicial complex

$$
D\left(\sigma^{j}\right)=c\left(1 \mathrm{k} \sigma^{j}\right),
$$

where $\mathrm{lk}$ denotes the link of in $V$, $^{\prime}$ denotes the first barycentric subdivision, and $c$ denotes the usual unreduced cone (unless $j=r$, when lk $\sigma=\varnothing$, in which case we understand $D\left(\sigma^{j}\right)$ to consist of a single point).

We claim that each $H^{j}$ can be endowed with the product regular structure $\overline{\boldsymbol{\sigma}}^{j} \subset D\left(\sigma^{j}\right)$. The figure below illustrates the situation for a triangulated 2-manifold by way of example:

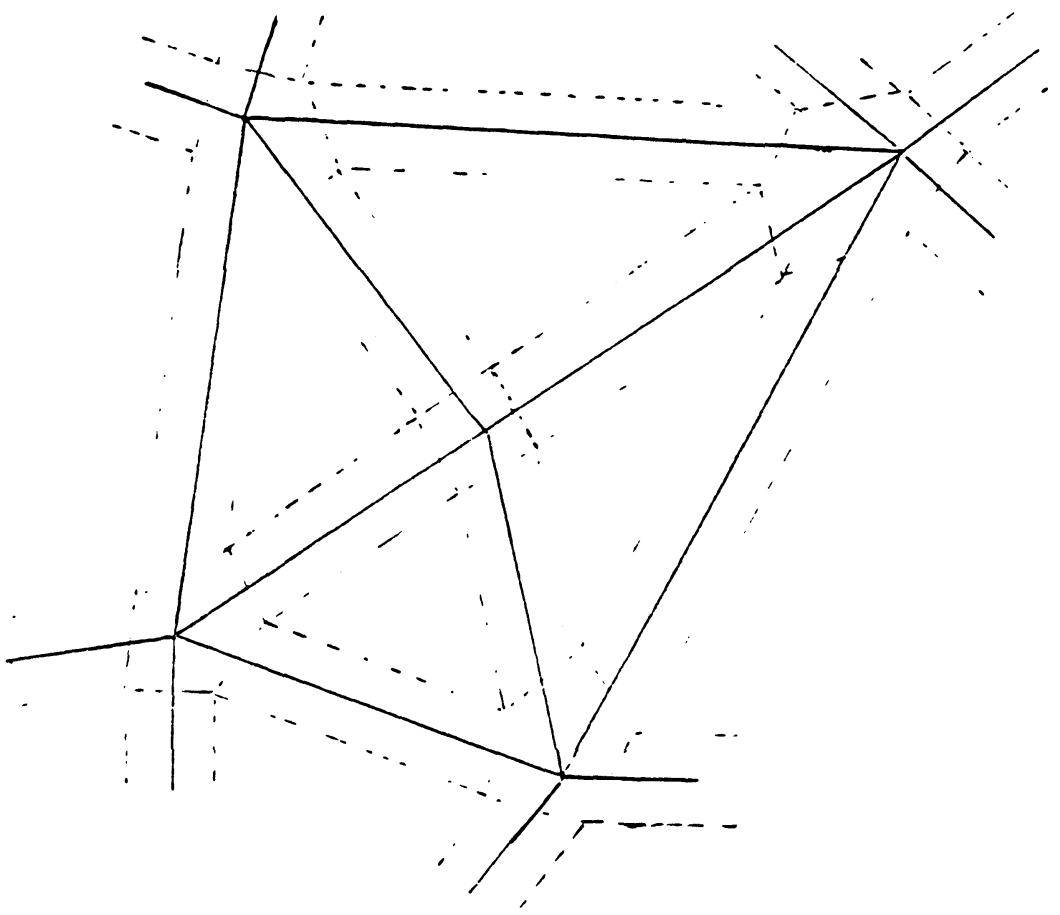

FIGURE 3.1 
In this diagram, the cells enclosed by solid lines are the simplices $\sigma$; cells enclosed by dotted lines are the handles $H(\sigma)$.

We must also make use of the following lemma.

3.2.3. Lemma. Let $A, B$ be $n$-dimensional Q.A.P.C.'s over $\mathbf{Z}$ with boundaries $\partial A, \partial B$, and let $f: \partial A \rightarrow \partial B$ be an orientation-reversing chain homotopy equivalence. Then there is a well-defined closed n-dimensional Q.A.P.C. $A \cup_{f} B$ over $\mathbf{Z}$. If $A, B$ are closed, with $\partial A=\partial B=0$, then $A \cup_{f} B=A \oplus B$ is just the direct sum of $A$ and $B$.

Moreover, Suppose $C_{l}(i=1,2)$ is an $(n+1)$-dimensional Q.A.P.C. with boundary and that $\partial C_{l}$ takes the form $A_{\imath} \cup_{f_{l}} B_{l}$ as above. Suppose $g$ is an orientation reversing chain homotopy equivalence $g:\left(A_{1}, \partial A_{1}\right) \rightarrow$ $\left(A_{2}, \partial A_{2}\right)$. Then we may form an $(n+1)$-dimensional Q.A.P.C. with boundary $C_{1} \cup_{g} C_{2}=C$ such that $\partial C=B_{1} \cup_{g^{\prime}} B_{2}$, where $g^{\prime}=$ $\left.f_{2} \circ g\right|_{\partial A_{1}} \circ f_{1}^{-1}: \partial B_{1} \rightarrow \partial B_{2}$.

Proof. This is a particular case of the glueing operation of $[\mathbf{1 4}, \S 3]$.

We now proceed with the construction of $T_{\alpha}$. Induction is involved both on the dimension $r$ of the manifold $V^{r}$ and on the dimension of handles. We may assume that QAPC $T_{\alpha}^{(l)}$ has been defined over $V^{(i)}=$ $\bigcup_{\operatorname{dim} \sigma \leq l} H(\sigma)$, where $T_{\alpha}^{(i)}$ has a boundary which may be constructed over $\partial V^{(i)}$. Consider now an $(i+1)$-handle $H\left(\sigma^{i+1}\right)$ with its stipulated cell structure. We form the Q.A.P.C. with boundary

$$
\beta(\sigma)=\alpha(\sigma) \times c(1 \mathrm{k} \sigma)^{\prime},
$$

a permissible construction in view of 3.2.1. Note that there is defined as well an $(i+1)$-ad of Q.A.P.C.'s with boundary, i.e, if we take $\sigma$ as a model of the standard simplex, we have for each face $\tau<\sigma$ the Q.A.P.C.

$$
\beta(\sigma, \tau)=\alpha(\tau) \times c(\mathrm{lk} \sigma)^{\prime} \subset \beta(\sigma) .
$$

Clearly

$$
\partial \beta(\sigma)=\alpha(\sigma) \times(1 \mathrm{k} \sigma)^{\prime} \subset \bigcup_{\tau<\sigma} \beta(\sigma, \tau) .
$$

As part of the inductive hypothesis we may assume that $\bigcup_{\tau<\sigma} \beta(\sigma, \tau) \subset$ $\partial T_{\alpha}^{(i)}$, where the latter Q.A.P.C. is decomposed as $\cup_{\tau<\sigma} \beta(\sigma, \tau) \cup C_{\sigma}$, with the components having common boundary $\bigcup_{\tau<\sigma} \alpha(\tau) \times(\mathrm{lk} \sigma)^{\prime}$.

Thus, by virtue of 3.2.3, we may form $T_{\alpha}^{(i)} \cup \beta(\sigma)$ along the identity on $\bigcup_{\tau<\sigma} \beta(\sigma, \tau)$. In effect we have extended the algebraic mock bundle over $V^{(i)}$ to one over $V^{(i)} \cup H\left(\sigma^{i+1}\right)$. 
In fact, we may do this for all $\sigma^{i+1}$ simultaneously, and thereby obtain $T_{\alpha}^{(i+1)}$ over $V^{(i+1)}$. [There still remain some technical points to complete the inductive step, but these are routine and we omit them.]

Clearly, after the $r$ th stage we achieve the construction of $T_{\alpha}^{(r)}=T_{\alpha}$ which is a closed Q.A.P.C., as desired.

This essentially completes the proof of the Assembly Lemma 3.2.0, although the relation to the original statement must be clarified somewhat. That is, the Q.A.P.C. $T_{\alpha}$ just constructed appears with a natural decomposition $\bigcup_{\sigma, \tau \leq \sigma} \alpha(\tau) \times c\left(\right.$ lk $\left.\sigma^{\prime}\right)$ (modulo identifications) rather than $\bigcup_{\sigma} \alpha(\sigma)$. The latter would seem to fit more naturally with the statement of 3.2.0. Thus the two points of view must be reconciled. This may be outlined as follows: for $\tau \leq \sigma$, set

$$
K(\sigma, \tau)=c(1 \mathrm{k} \tau)^{\prime} \cap \sigma .
$$

(Here, we think of $c(1 \mathrm{k} \tau)^{\prime}$ as a subspace of $\operatorname{st}(\tau)$ in the natural way; so, in particular, $K(\sigma, \sigma)$ consists of a single point.) Now set

$$
\gamma(\sigma)=\bigcup_{\tau \leq \sigma} \alpha(\tau) \times K(\sigma, \tau)
$$

and $\dot{\gamma}(\sigma)=\bigcup_{\tau<\sigma} \alpha(\tau) \times[K(\sigma, \tau) \cap \dot{\sigma}]$. We claim that $(\gamma(\sigma), \dot{\gamma}(\sigma))$ is canonically chain homotopy equivalent to $(\alpha(\sigma), \partial \alpha(\sigma))$; in fact, for $j=\operatorname{dim} \sigma$, the $j$-ad $\left(\gamma(\sigma),\{\gamma(\tau)\}_{\tau<\sigma}\right)$ is canonically chain homotopy equivalent to $\left(\alpha(\sigma),\{\alpha(\tau)\}_{\tau<\sigma}\right)$. Thus, allowing for consistent replacement of components by chain homotopy equivalent ones, we have that $\bigcup_{\sigma} \alpha(\sigma)$ is chain equivalent to $\bigcup_{\sigma} \gamma(\sigma)=T_{\alpha}$.

Returning, finally, to the statement of the Assembly Lemma 3.2.0 per se, we instate for the assignment $\sigma \mapsto \alpha(\sigma)$ on the manifold $V^{r}$ the specific assignment $\sigma \mapsto R_{\sigma}$ on $M^{n+k}$ as in the context of 3.2.0. We thereby obtain part (i) of the lemma.

Routine relativization of the arguments above yield (ii) and (iii) as well.

Returning now to the specific situation of Lemma 1.22 we have an RT map $g: W \cup c \partial W \rightarrow T\left(\nu^{k}\right)$, where $W$ is a regular neighborhood of the PD-space $X^{n}$ in $\mathbf{R}^{n+k}$, and $\nu^{k}$ is the Spivak normal $(k-1)$-spherical fibration for $X$. We may assume that $g^{-1}\left(\mathscr{M}\left(\nu^{k}\right)\right) \subset W-\partial W \subset W \cup$ $c \partial W$. Thus on the triangulated $W$ we have the RT map $g_{0}=\left.g\right|_{W}$. For each simplex $\sigma$ we have the quadratic Poincaré pair over $\mathbf{Z} R_{\sigma}$ with $\partial R_{\sigma}=\bigcup_{\tau \subseteq \partial \sigma} R_{\tau}$, and we also have quadratic Poincaré pairs $J_{\sigma}$ with $\partial J_{\sigma}=R_{\sigma} \cup \bigcup_{\tau \subseteq \partial \sigma} J_{\tau}$. By the assembly lemma above we have a symmetric Poincaré complex over $\mathbf{Z} \Gamma_{*}$ together with a chain map $f: \Gamma_{*} \rightarrow C_{*}(X)$. The quadratic construction of $[15, \S 1]$ determines an $n$-dimensional QAPC 
over $\mathbf{Z}\left(C\left(f^{!}\right), \psi\right)$ with $C\left(f^{!}\right)$the algebraic mapping cone of the Umkehr chain map $f^{!}: C(X) \simeq C(X)^{n-*} \stackrel{f^{*}}{\rightarrow} \Gamma^{n-*} \cong \Gamma_{*}$. However, this alone will not suffice to yield a "non-simply-connected" surgery obstruction element in $L_{n}\left(\pi_{1}(X)\right)$ as required for 1.22 . We therefore pass to the universal covering space $p: \tilde{W} \rightarrow W$ with the induced triangulation. Clearly, for each simplex $\sigma$ of $\tilde{W}$ we have $R_{\sigma}=R_{p \sigma}, J_{\sigma}=J_{p \sigma}$ with $\partial R_{\sigma}=\bigcup_{\tau \subseteq \dot{\sigma}} R_{\tau}$, $\partial J_{\sigma}=R_{\sigma} \cup \bigcup_{\tau \subseteq \sigma} J_{\tau}$. The fundamental group $\pi_{1}(X)=\pi_{1}(W)$ acts on $\left\{R_{\sigma}\right\},\left\{J_{\sigma}\right\}$ by the obvious permutations $\alpha: R_{\sigma} \rightarrow R_{\alpha \sigma}, \alpha: J_{\sigma} \rightarrow J_{\alpha \sigma}$ $\left(\alpha \in \pi_{1}(X)\right)$. Thus we have, by an easy modification of the assembly lemma, an $(n-1)$-dimensional QAPC over $\mathbf{Z}\left[\pi_{1}(X)\right]$ measuring the failure of $g^{-1}\left(\eta\left(\nu^{k}\right)\right)$ to satisfy Poincaré duality over $\mathbf{Z}\left[\pi_{1}(X)\right]$. Moreover $\bigcup_{\sigma \subseteq \tilde{W}} J_{\sigma}$ is now a null-bordism of $\bigcup_{\sigma \subseteq \tilde{W}} R_{\sigma}$ in the category of QAPCs over $\mathbf{Z}\left[\pi_{1}(X)\right]$ and we thus obtain $\tilde{\Gamma}=\bigcup_{\sigma \subseteq \tilde{W}} \Gamma_{\sigma}$, an $n$-dimensional symmetric Poincaré complex over $\mathbf{Z}\left[\pi_{1}(X)\right]$ with a chain map $f: \Gamma_{*} \rightarrow C(\tilde{X})$, such that there is defined a (based) $n$-dimensional QAPC over $\mathbf{Z}\left[\pi_{1}(X)\right]$ $\left(C\left(f^{!}\right), \psi\right)=\tilde{\Phi}_{g}$. The bordism class $\left[\tilde{\Phi}_{g}\right]$ is the surgery obstruction in $L_{n}\left(\pi_{1}(X)\right)$.

Clearly, if $g: W \rightarrow T\left(\nu^{k}\right)$ is in fact PT then $R_{\sigma}=0, J_{\sigma}=0(\sigma \in \tilde{W})$ and $f=g \mid: \Gamma_{*}=C(\tilde{Y}) \rightarrow C(\tilde{X})$ with $Y=g^{-1}\left(\mathscr{M}\left(\nu^{k}\right)\right)$. Moreover $\tilde{\Phi}_{g}$ is just the quadratic kernel of the normal map of P.D. spaces $Y \rightarrow X$. So, in particular, if $Y \rightarrow X$ is a simple homotopy equivalence then the quadratic complex $\tilde{\Phi}_{g}$ is 0 (up to equivalence) and $\left[\tilde{\Phi}_{g}\right]=0 \in L_{n}\left(\pi_{1}(X)\right)$.

Thus we have proved that part of 1.22 which asserts that the RT map $g:(W \cup c \partial W, c \partial W) \rightarrow\left(T\left(\nu^{k}\right),{ }^{*}\right)$ gives rise to a specific $n$-dimensional QAPC $\tilde{\Phi}_{g}$. That a specific simplex-wise RT homotopy $G$ of two such maps $g_{0}, g_{1}: W \cup c \partial W \rightarrow T\left(\nu^{k}\right)$ gives rise to a $\mathbf{Z}\left[\pi_{1}(X)\right]$-QAPC bordism $\tilde{\Phi}_{G}$ from $\tilde{\Phi}_{g_{0}}$ to $\tilde{\Phi}_{g_{1}}$ is a straightforward relativization of the construction of $\tilde{\Phi}_{g}$.

\subsection{Appendix - The intrinsic transversality obstruction map and struc-} ture theory. In this section, without giving full details, we shall reinterpret Theorem 1.27, expanding it somewhat.

Consider an (orientable) PD-space $X^{n}$, and a triangulated regular neighborhood $W^{n+1} \subset \mathbf{R}^{n+k}$. By elementary general position considerations we may assume that $\left(X_{\sigma}, X_{\dot{\sigma}}\right)=(X \cap \sigma, X \cap \dot{\sigma})$ is an $(n-j)$ dimensional $\nu(X)$-normal pair (in fact, a normal pair for the trivial spherical fibration) whenever $\sigma$ is an $(n+k-j)$-dimensional simplex of $W^{n+k}$. (Of course, $X_{\sigma} \neq \varnothing$ if $\sigma \subseteq \partial W$.) Thus, for each simplex $\sigma$ of dimension $i+k$ we obtain an $(i-1)$-dimensional QAPC with boundary $\Phi_{\sigma}$ (over the ring $\mathbf{Z}$ ), in fact an $(i+k)$-ad $\left\{\Phi_{\tau}\right\}_{\tau \subseteq \sigma}$, measuring the failure 
of $\left(X_{\sigma}, X_{\dot{\sigma}}\right)$ to be a simple-coefficient Poincaré duality pair. In particular, $\Phi_{\dot{\sigma}}=\bigcup_{\tau \subseteq \dot{\sigma}} \Phi_{\tau}$ is a closed $(i-2)$-dimensional QAPC measuring the failure of $X_{\dot{\sigma}}$ to be a simple Poincare duality space. If $\Phi_{\dot{\sigma}}=0$ then $\Phi_{\sigma}$ is a closed QAPC. If $X$ admits a codimension $k$ intrinsic transversality structure, it follows that $X_{\sigma}$ can be chosen so that $\Phi_{\sigma}=0$.

Note that $U_{\sigma} \Phi_{\sigma}$ is a closed $(n-1)$-dimensional QAPC over $\mathbf{Z}$ which measures the failure of $X^{n}$ to satisfy simple coefficient Poincaré duality, hence, up to chain homotopy $\cup_{\sigma} \Phi_{\sigma}=0$. Moreover, by the assembly procedure of 3.2, we may enrich the structure of $U_{\sigma} \Phi_{\sigma}$ to make it the image of a $Z\left[\pi_{1}(X)\right]$ QAPC, i.e. we may take $\bigcup_{\sigma \subseteq \tilde{W}} \tilde{\Phi}_{\sigma}$, where $\tilde{\Phi}_{0}=\tilde{\Phi}_{p \sigma}$, with $p: \tilde{W} \rightarrow W$ the universal cover. Of course $\bigcup_{\sigma \subseteq \tilde{W}} \tilde{\Phi}_{0}$ measures the failure of $X^{n}$ to satisfy $\mathbf{Z}\left[\pi_{1}(X)\right]$-Poincaré duality (i.e. to be a PD-space) and hence this also vanishes up to chain homotopy equivalence over $\mathbf{Z}\left[\pi_{1}(X)\right]$.

We now view the assignment $\sigma \mapsto \Phi_{\sigma}$ as a simplicial map $W / \partial W \stackrel{N}{\rightarrow} \mathbf{L}_{-k-1}(\{1\})$. Where the normal pairs $\left(X_{\sigma}, X_{\dot{\sigma}}\right)$ are PD pairs (i.e. $X$ has an intrinsic transversality structure) the map is the trivial one to the standard base point (if we think of the "base point" as the contractible subcomplex of $\mathbf{L}_{-k-1}(\{1\})$ having one simplex in each dimension, corresponding to the QAPC 0 of the appropriate formal dimension).

More generally, we see the extent to which the map $N$ is an invariant of the PD-space $X^{n}$, noting that $N$ is well-defined up to homotopy, even allowing for changes in the triangulation of $W$. In fact, the homotopy class of $N$ is precisely the obstruction to the reducibility of $\nu(X)$ to a topological bundle, i.e. $N$ is homotopically trivial if and only if $\nu(X)$ admits a topological structure and, moreover, the topological reductions of $\nu(X)$ are in 1-1 correspondence with the homotopy classes of nullhomotopies of $N$.

Furthermore, we may read off, so to speak, the surgery obstruction arising from a particular topological bundle reduction of $\nu(X)$ as follows. If we have $N$ (as a specific simplicial map) and a null-homotopy $L$ : $W / \partial W \times I \rightarrow \mathbf{L}_{-k-1}(\{1\})$ defined up to homotopy rel $W / \partial W \times \dot{I}$, then we may as well assume that $L$ is simplicial with respect to some triangulation of $W \times I$ extending the given one on $W \times\{0\}$. Thus for each simplex $\sigma^{j}$ of $W \times I$ we have a QAPC over $\mathbf{Z} \Phi_{\sigma}$ of dimension $j-k-1$, with $\Phi_{\sigma}=0$ if $\sigma \subseteq \partial W \times I \cup W \times\{1\}$. Passing to the universal covering $p: \tilde{W} \times I \rightarrow W \times I$ we have $\Phi_{\sigma}=\Phi_{p \sigma}$ and $\bigcup_{\sigma \subseteq \tilde{W}} \Phi_{\sigma}$ admits a $\pi_{1}(X)$-action and thus we may assemble a QAPC $s(L)$ of dimension $n$ over $\mathbf{Z}\left[\pi_{1}(X)\right]$. Note that $\partial s(L)=0$ since $\partial s(L)=\bigcup_{\sigma \subseteq \tilde{W} \times\{0\}} \Phi_{\sigma} \cup \cup_{\sigma \subseteq \tilde{W} \times\{1\}}$ $-\Phi_{\sigma}=0$. The first summand being the (vanishing) global obstruction to 
the $\mathbf{Z}\left[\pi_{1}(X)\right]$-Poincare duality of $X$, and the second being trivially 0 . Thus $s(L)$ is a closed QAPC over $Z\left[\pi_{1}(X)\right]$ and it is easily seen that the bordism class $[s(L)] \in L_{n}\left(\pi_{1}(X)\right)$ is independent of the particular choice of $L$ within its homotopy class rel $W / \partial W \times \dot{I}$. In fact $[s(L)]$ is precisely the surgery obstruction associated to the topological bundle reduction of $\nu(X)$ corresponding to $L$.

We may further sharpen this observation by defining the following set, having fixed $N$. Consider pairs consisting of specific simplicial nullhomotopies $L$ of $N$, together with specific $(n+1)$-dimensional quadratic Poincaré pairs over $\mathrm{Z}\left[\pi_{1}(X)\right] S$ such that $\partial S=s(L)$. Call two pairs $\left(L_{0}, S_{0}\right),\left(L_{1}, S_{2}\right)$ equivalent whenever there is a homotopy $H$ from $L_{0}$ to $L_{1}$, in which case the assembly procedure applied to $H$ gives an $(n+1)$ dimensional quadratic Poincaré pair over $\mathbf{Z}\left[\pi_{1}(X)\right] T_{H}$ with boundary $\partial T_{H}=s\left(L_{0}\right) \cup-s\left(L_{1}\right)$ (i.e. $T_{H}$ is a bordism from $s\left(L_{0}\right)$ to $s\left(L_{1}\right)$ ). Thus we obtain a closed QAPC by adjoining $S_{0}$ and $S_{1}$ to $T_{H}$, i.e. we get $U_{H}=-S_{0} \cup_{-s\left(L_{0}\right)} T_{H} \cup_{s\left(L_{1}\right)} S_{1}$. We now require, as part of the definition of equivalence between $\left(L_{0}, S_{0}\right)$ and $\left(L_{1}, S_{1}\right)$, that $H$ may be chosen so that $U_{H}$ is null bordant as an $(n+1)$-dimensional QAPC over $\mathbf{Z}\left[\pi_{1}(X)\right]$ (i.e. $\left.\left[U_{H}\right]=0 \in L_{n+1}\left(\pi_{1}(X)\right)\right)$. This completes the definition of the equivalence relation.

3.3.1. THEOREM. The structure set $\mathscr{S}_{\text {TOP }}(X)$ of $X$ is in 1-1 correspondence with the set of equivalence classes $(L, S)$.

As a corollary we see that $\mathscr{S}_{\text {TOP }}(X)$, if non-empty, is endowed with an abelian group structure with respect to which the classical structure sequence

$$
\cdots \rightarrow L_{n+1}\left(\pi_{1}(X)\right) \rightarrow \mathscr{S}_{\text {TOP }}(X) \rightarrow[X, G / \text { TOP }] \rightarrow L_{n}\left(\pi_{1}(X)\right)
$$

becomes an exact sequence of abelian groups (cf. [10], [13]). First of all, pick a base topological manifold structure on $X$, and so regard $\mathscr{S}_{\text {TOP }}(X)$ as the set of "difference elements". With reference to this specific topological structure on $X$ we obtain an intrinsic transversality structure (of codimension $k$ ) and hence we see that the map $N: W / \partial W \rightarrow \mathbf{L}_{-k-1}(\{1\})$ may be taken to be the trivial one. Thus the set $\mathscr{S}_{\text {TOP }}(X)$ is interpreted to mean the set of equivalence classes of pairs $(L, S)$ where $L$ is a selfhomotopy of the trivial map $N$, viz. $L:(W \times I, W \times \dot{I} \cup \partial W \times I) \rightarrow$ $\left.\mathbf{L}_{-k-1}(\{1\}),{ }^{*}\right)$ and $S$ is a QAPC over $\mathbf{Z}\left[\pi_{1}(X)\right]$ with $\partial S=s(L)$. We define a group operation on this set, i.e. $\left[\left(L_{0}, S_{0}\right)\right]+\left[\left(L_{1}, S_{1}\right)\right]$ is defined by the pair $(L, S)$ where $L$ is the concatenation of the homotopies $L_{0}, L_{1}$ 
(so that $s(L)=s\left(L_{0}\right) \cup s\left(L_{1}\right)$ ) and $S=S_{0} \cup S_{1}$. We are justified in using additive notation because this group operation is commutative (essentially because $W / \partial W$ is a suspension). The existence of inverses and the verification of the associative law are trivialities best left to the reader.

It remains to describe the maps

$$
L_{n+1}\left(\pi_{1}(X)\right) \rightarrow \mathscr{S}_{\text {TOP }}(X), \quad \mathscr{S}_{\text {TOP }}(X) \rightarrow[X, G / \mathrm{TOP}],
$$

whereupon it will be observed that these are abelian group homomorphisms.

First of all, if $a \in L_{n+1}\left(\pi_{1}(X)\right)$ is represented by the closed QAPC $S$ over $\mathbf{Z}\left[\pi_{1}(X)\right]$ then the map $L_{n+1}\left(\pi_{1}(X)\right) \rightarrow \mathscr{S}_{\text {TOP }}(X)$ sends $a$ to the pair $\left({ }^{*}, S\right)$, where ${ }^{*}$ is the trivial self-homotopy of $N$ (thus $s\left(^{*}\right)=0$ ) and $S$ is $a$ regarded as a null-bordism of $s\left(^{*}\right)=0$. Clearly, this map is independent of the choice of representatives, and is a homomorphism.

The map $\mathscr{S}_{\text {TOP }}(X) \rightarrow[X, G /$ TOP $]$ has already been referred to at least in passing, previously. The point is that maps $\Sigma(W / \partial W)=$ $T\left(\nu^{k}(X) \oplus \varepsilon\right) \rightarrow \mathbf{L}_{-k}(\{1\})$ are in 1-1 correspondence with the homotopy classes of maps $X \rightarrow G /$ TOP in a way that makes the correspondence an isomorphism with respect to the usual group structure on $[X, G / \mathrm{TOP}]$. In other words we may think of $[X, G / \mathrm{TOP}]$ as the group of homotopy equivalence classes of self-homotopies $L$ of the trivial map $N: W / \partial W \rightarrow$ $\mathbf{L}_{-k-1}(\{1\})$, and so the map $\mathscr{S}_{\text {TOP }}(X) \rightarrow[X, G /$ TOP $]$ may be described $[(L, S)] \rightarrow[L]$.

Exactness is easily seen. Clearly the composite $L_{n+1}\left(\pi_{1}(X)\right) \rightarrow$ $\mathscr{S}_{\text {TOP }}(X) \rightarrow[X, G / \mathrm{TOP}]$ is trivial. Moreover, if $[(L, S)]$ goes to 0 , i.e. $L$ is homotopic to *, then we may choose a homotopy $H$ of $L$ to ${ }^{*}$, apply the assembly procedure to obtain $T_{H}$ with $\partial T_{H}=-S(L)$, and find that $(L, S)$ is equivalent to $\left(*, T_{H} \cup-S\right)$, and thus $[(L, S)]$ is the image of $\left[T_{H} \cup-S\right] \in L_{n+1}\left(\pi_{1}(X)\right)$.

3.4. The combinatorial intrinsic transversality obstruction. In conclusion, we relate the results above to the total surgery obstruction of Ranicki [13], and further indicate a combinatorial construction of the same for triangulated Poincaré duality spaces.

Recall the homotopy functor $\mathscr{S}_{*}(K)$ defined for any finite $\mathrm{CW}$ complex $K . \mathscr{S}_{*}$ is a non-excisive homology theory, meaning that it satisfies all the axioms for a homology theory, save dimension and excision. Briefly, this theory is described as follows: The spaces $\mathbf{L}_{-i}(\{1\})$ form a spectrum whose homology theory we denote by $[\mathbf{L}(\{1\})]_{*}$. The notion of assembly map extends so as to define a homomorphism $[\mathbf{L}(\{1\})]_{i}(K) \rightarrow L_{i}\left(\pi_{1}(K)\right)$. One way to see this is to replace $[\mathbf{L}(\{1\})]_{i}(K)$ 
by the $S$-dually isomorphic cohomology group $[\mathbf{L}(\{1\})]^{k}(W, \partial W)$, where $W$ is a regular neighborhood of $K$ in $\mathbf{R}^{i+k}, k$ large. Thus any element of $[\mathbf{L}(\{1\})]_{i}(K)$ may be represented by a map $f: W / \partial W \rightarrow \mathbf{L}_{-k}(\{1\})$ which may be taken to be simplicial. The assembly Lemma 3.2.0 now yields an $i$-dimensional Q.A.P.C. $Q(f)$ over $\mathbf{Z}\left[\pi_{1}(W)\right]=\mathbf{Z}\left[\pi_{1}(K)\right]$ whose bordism class is thus an element of $L_{i}\left(\pi_{1}(K)\right)$. By definition, $\mathscr{S}_{i}(K)$ is the "cofiber" of this homomorphism, i.e. it fits into an exact sequence

$$
\cdots \rightarrow[\mathbf{L}(\{1\})]_{i}(K) \rightarrow L_{i}\left(\pi_{1}(K)\right) \rightarrow \mathscr{S}_{l}(K) \rightarrow[\mathbf{L}(\{1\})]_{i-1}(K) \rightarrow \cdots
$$

More specifically, $\mathscr{S}_{i}(K)$ may be defined to be the group of equivalence classes of pairs $(f, U)$ where $f$ is a simplicial map $W / \partial W \rightarrow \mathbf{L}_{-i+1}(\{1\})$ and $U$ is an $i$-dimensional Q.A.P.C. over $\mathbf{Z}\left[\pi_{1}(K)\right]$ with boundary $Q(f)$. The pairs $\left(f_{1}, U_{1}\right),\left(f_{2}, U_{2}\right)$ are equivalent if there exists a homotopy $F$ : $f_{1} \sim f_{2}$ such that $U_{1} \cup Q(F) \cup-U_{2}$ bounds an $(i+1)$-dimensional Q.A.P.C. over $\mathbf{Z}\left[\pi_{1}(K)\right]$.

Consider the special case of a PD-space $X^{n}$ with regular neighborhood $W^{n+k}$ in $\mathbf{R}^{n+k}$. We have seen above that there is an assignment $\sigma \mapsto \phi_{\sigma}$ of Q.A.P.C.'s $\phi_{\sigma}$ to the simplices $\sigma$ of $W$ (once the inclusion $X \subset W$ is in general position) defining a simplicial map $f: W / \partial W \rightarrow$ $\mathbf{L}_{-k-1}(\{1\})$. The union $\cup_{\sigma} \phi_{\sigma}=Q(f)$ is the obstruction to $X^{n}$ being a PD-space, 0 by hypothesis, and there is a canonical bordism $U(f)$ of $Q(f)$ to 0 (namely, the 0 bordism). The element $s(X)=[(f, U(f))] \in$ $\mathscr{S}_{n}(X)$ is independent of $f$.

3.4.0. THEOREM. The element $s(X) \in \mathscr{S}_{n}(X)$ is the total surgery obstruction of Ranicki [13], with $s(X)=0$ if (and for $n \geq 5$ only if) $X$ admits a topological manifold structure.

In passing we note that the image of $s(X)$ in $[\mathbf{L}(\{1\})]_{n-1}(X)$ is the obstruction to putting a topological block-bundle structure on the Spivak normal fibration $\nu_{X}: X \rightarrow B G(k)$, which was first obtained by Levitt [6] (as an element of the $S$-dually isomorphic reduced cohomology group $\left.[\overline{\mathbf{L}(\{1\})}]^{k+1}\left(T\left(\nu_{X}\right)\right)\right)$.

We note the further fact that the characterization of the structure set $\mathscr{S}_{\text {TOP }}(X)$ (when $X$ admits at least one topological manifold structure) given in 3.3 .1 above may be similarly interpreted to identify $\mathscr{S}_{\text {TOP }}(X)$ with $\mathscr{S}_{n+1}(X)$.

A further refinement is to be seen in the following "combinatorial formula" for the total surgery obstruction of a triangulated PD-space $X^{n}$. We assume provisionally that $X^{n}$ is orientable and that the triangulation 
of $X$ has the following property: given a simplex $\sigma$ of the triangulation, st $\boldsymbol{\sigma}$ is an $n$-dimensional complex with $H_{n}(\operatorname{st} \sigma, \dot{\sigma} * \operatorname{lk} \boldsymbol{\sigma})=\mathbf{Z}$ and the collapsing map $X \rightarrow$ st $\sigma / \dot{\sigma} * \mathrm{lk} \sigma$ is of degree 1 .

We next remark that the homology theory $[\mathbf{L}(\{1\})]_{i}(K)$ has the following characterization when the finite complex $K$ has a specific simplicial triangulation: An $\mathbf{L}(\{1\})$-cycle $\psi$ of dimension $i$ is an assignment to each simplex $\sigma$ of $K$ of an $i$-dimensional Q.A.P.C. $\psi_{\sigma}$ with boundary $\partial \psi_{\sigma}=U_{\tau>\sigma} \psi_{\tau}$. Bordism of cycles is defined in an obvious way, based on the notion of bordism among Q.A.P.C.'s. It is, moreover, true that given such a cycle $\psi, Q(\psi)=\bigcup_{\sigma \subset k} \psi_{\sigma}$ is a closed $i$-dimensional Q.A.P.C.; in fact, by an extension of the Assembly Lemma, $Q(\psi)$ has, in a natural way, the structure of a Q.A.P.C. over $\mathbf{Z}\left[\pi_{1}(K)\right]$.

We may thus characterize $\mathscr{S}_{i}(K)$ as bordism classes of pairs $(\psi, U)$ where $\psi$ is an $(i-1)$-dimensional $\mathbf{L}(\{1\})$-cycle on $K$ and $U$ is an $i$-dimensional Q.A.P.C. over $\mathbf{Z}\left[\pi_{1}(K)\right]$ with $\partial U=Q(\psi)$.

Given a triangulated P.D. space $X^{n}$, there is a natural $(n-1)$ dimensional $\mathbf{L}(\{1\})$-cycle $\psi(X)$ as follows: Given $\sigma^{k}, \psi_{\sigma}$ is the $(n-k-1)$ dimensional Q.A.P.C.-with-boundary measuring the failure of the pair $(c \mathrm{k} \sigma, 1 \mathrm{k} \sigma)$ to be a Poincaré pair, which is, to all intents and purposes, the failure of $\mathrm{lk} \sigma$ to be a homology $(n-k-1)$-sphere. We claim that $\psi(X)$ assembles to an $(n-1)$-dimensional Q.A.P.C. $Q(\psi(X))=Q(X)$ over $\mathbf{Z}\left(\pi_{1}(X)\right)$ which is chain-homotopy equivalent to 0 inasmuch $Q(X)$ measures the global failure of $X$ to be Poincaré, and is thus 0 by hypothesis. Let $T$ denote the trivial bordism between $Q(X)$ and 0 .

3.4.1. COROLLARY. The element $[Q(X), T] \in \mathscr{S}_{n}(X)$ is the total surgery obstruction $s(X)$.

The proof may be sketched as follows: Note first that $X$ may be decomposed into "pseudo-cells" dual to the simplices of $X$. In this decomposition we have, corresponding to each simplex $\sigma$, the subcomplex $e_{\sigma}$ of $X^{\prime}$ canonically isomorphic to $c(1 \mathrm{k} \sigma)^{\prime}$. (By way of example, if $X$ is a combinatorial manifold, $e$ is the usual dual cell.)

Consider a regular neighborhood $W^{n+k}$ ( $k$ large) of $X$ in $\mathbf{R}^{n+k}$. We claim that $W^{n+k}$ may be stratified so that, to each simplex $\sigma^{j}$ of $X$ there corresponds a submanifold $W_{\sigma} \subset W$ of dimension $n+k-j$ so that under the embedding $X \subset W$ we have $X \cap W_{\sigma}=e_{\sigma}$. We then triangulate $W$ so that each such $W_{\sigma}$ is a subcomplex. Let, for each simplex $\tau$ of $W, \phi_{\tau}$ denote the Q.A.P.C. over $\mathbf{Z}$ measuring the failure of $X \cap \tau, X \cap \dot{\tau}$ to satisfy simple-coefficient Poincaré duality, thus yielding a family $\left\{\phi_{\tau}\right\}$ of 
Q.A.P.C.'s as in the beginning of this section. Then $Q=\cup_{\tau} \phi_{\tau}, U=0$ bordism is, by 3.4.0, a representative of the total surgery obstruction $s(X) \in \mathscr{S}_{n}(X)$. But note that $\bigcup_{\tau \subset W_{\sigma}} \phi_{\tau}$ is canonically chain-homotopy equivalent as a Z-Q.A.P.C. to $\psi_{\sigma}$. Thus, as a $\mathbf{Z}\left[\pi_{1}(X)\right]$-Q.A.P.C., $Q$ may be identified with $Q(X)$ and $U$, of course, with $T$. Hence $[Q(X), T]=$ $s(X)$ as required.

By way of concluding remarks we note that the condition assumed on the triangulation of $X$ is not very restrictive. It will follow from L. Jones' theory of patch structures on P.D. spaces [3] that P.D. spaces of dimension $\geq 5$ always admit triangulations of the type specified. On the other hand, it can be shown that the "local combinatorial formula" for $s(X)$ is still applicable even for triangulations of a P.D. space not meeting this condition. This extension, however, requires a more permissive definition of the notion of "normal space" than has been used in this paper. We also note that given a homotopy equivalence $f: M \rightarrow N$ of two closed PL manifolds (or, more generally, integral homology manifolds), a similar construction on the geometric mapping cylinder of $f$ yields an analogous "local combinatorial formula" for $[f] \in \mathscr{S}_{n+1}(X)=\mathscr{S}_{\text {TOP }}(X)$.

More complete proofs of the results of this section will appear in subsequent work of the second author where the algebraic and categorical foundations necessary to supply the missing details will be established.

\section{REFERENCES}

[1] G. Brumfiel and J. Morgan, Homotopy-theoretic consequences of N. Levitt's obstruction theory to transversality for spherical fibrations Pacific J. Math., 67 (1976), 1-100.

[2] S. Buoncristiano, C. P. Rourke and B. J. Sanderson, A geometric approach to homology theory, London Math. Soc. Lecture Note Series 18 (1976).

[3] L. Jones, Patch Spaces: a geometric representation for Poincaré duality spaces, Ann. of Math., 97 (1973), 306-343.

[4] N. Levitt, Poincaré duality cobordism, Ann. of Math., 96 (1972), 211-244.

[5] $\quad$ A necessary and sufficient condition for fibering a manifold, Topology, 14 (1975), 229-236.

[6] On a $\sum$-spectrum related to G/Top, Houston J. Math., 3 (1977), 481-493.

[7] N. Levitt and J. Morgan, Transversality structures and PL structures on spherical fibrations, Bull. Amer. Math. Soc., 78 (1974), 1064-1068.

[8] A. S. Mishchenko and Yu. P. Solovev, A classifying space for hermitian K-theory Trudy Sem. Vect. and Tensor Anal., 18 (1976), 140-168.

[9] F. Quinn, A geometric formulation of surgery, Proc. 1969 Georgia Conference on the Topology of Manifolds, Markham Press, (1970), 500-512.

[10] $\quad, B_{(\widetilde{T O P})_{n}}$ and the surgery obstruction I. Bull. Amer. Math. Soc., 77 (1971), 596-600.

[11] _ Surgery on Poincaré and normal spaces, ibid. 78 (1972), 262-267.

[12] A. Ranicki, An algebraic formulation of surgery, Trinity College, Cambridge fellowship dissertation (1972). 
[13] The total surgery obstruction, Proceedings of 1978 Århus Conference on Algebraic Topology, Springer Lectures Notes, 763 (1979), 275-316.

[14] The algebraic theory of surgery I. Foundations, Proc. L. M. S., (3) 40 (1980), 87-192.

[15] _ II. Applications to topology, ibid., (1980), 193-283.

[16] Exact sequences in the algebraic theory of surgery, Mathematical Notes 26, Princeton (1981).

[17] M. G. Scharlemann, Transversality theories at dimension 4, Invent. Math., 33 (1976), 1-14.

[18] D. Sullivan, Geometric topology I. Localization, periodicity and Galois symmetry, MIT notes (1970).

Received December 7, 1981.

RUTGERS UNIVERSITY

NEW BRUNSWICK, NJ 08903, USA

AND

UNIVERSITY OF EDINBURGH

EDINBURGH EH9 3JZ, ScOTLAND 


\section{PACIFIC JOURNAL OF MATHEMATICS EDITORS}

V. S. VARADARAJAN

(Managing Editor)

University of California

Los Angeles, CA 90024

Herbert Clemens

University of Utah

Salt Lake City, UT 84112

R. FINN

Stanford University

Stanford, CA 94305
ROBION KIRBY

University of California

Berkeley, CA 94720

C. C. MOORE

University of California

Berkeley, CA 94720

HAROLD STARK

University of California, San Diego La Jolla, CA 92093

\section{ASSOCIATE EDITORS}
R. ARENS
E. F. BECKENBACH
B. H. NEUMANN
F. WOLF
K. YosHIDA (1906-1982)

\section{SUPPORTING INSTITUTIONS}

UNIVERSITY OF ARIZONA

UNIVERSITY OF BRITISH COLUMBIA

CALIFORNIA INSTITUTE OF TECHNOLOGY

UNIVERSITY OF CALIFORNIA

MONTANA STATE UNIVERSITY

UNIVERSITY OF NEVADA, RENO

NEW MEXICO STATE UNIVERSITY

OREGON STATE UNIVERSITY
UNIVERSITY OF OREGON UNIVERSITY OF SOUTHERN CALIFORNIA

STANFORD UNIVERSITY

UNIVERSITY OF HAWAII

UNIVERSITY OF TOKYO

UNIVERSITY OF UTAH

WASHINGTON STATE UNIVERSITY

UNIVERSITY OF WASHINGTON 


\section{Pacific Journal of Mathematics}

Vol. 129, No. $1 \quad$ May, 1987

Aldridge Knight Bousfield, Uniqueness of infinite deloopings for

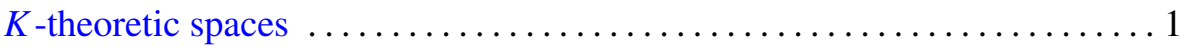

Mark Gregory Davidson, The harmonic representation of $U(p, q)$ and its

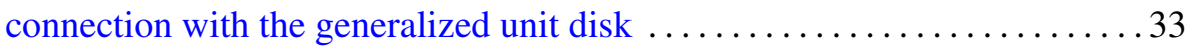

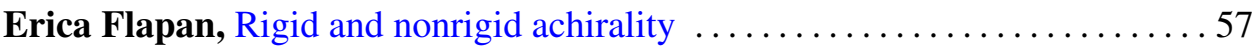

Peter Abraham Greenberg, Pseudogroups of $C^{1}$ piecewise projective

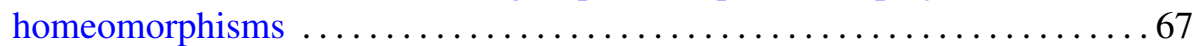

Peter Martin Knopf, Maximal functions on the unit $n$-sphere $\ldots \ldots \ldots \ldots 77$

Norman Jay Levitt and Andrew Ranicki, Intrinsic transversality

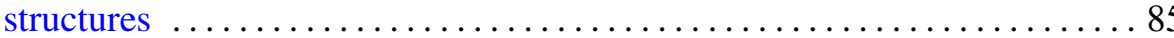

Susan Szczepanski, Invariant submanifolds of free cyclic actions on

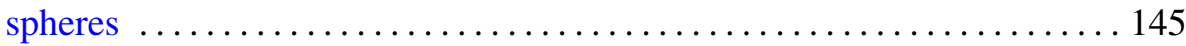

Kazimierz Szymiczek, Generalized rigid elements in fields . . . . . . . . 171

Domingo Toledo, Nonexistence of certain closed complex geodesics in the

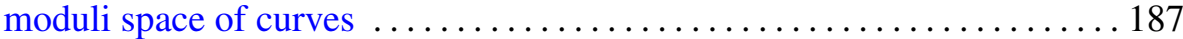

Graham H. Williams, The best modulus of continuity for solutions of the

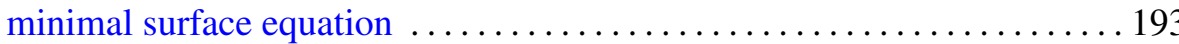

